\title{
Structural determinants of murine leukemia virus reverse transcriptase that are important for template switching, fidelity, and drug resistance
}

\author{
Evguenia S. Svarovskaia \\ West Virginia University
}

Follow this and additional works at: https://researchrepository.wvu.edu/etd

\section{Recommended Citation}

Svarovskaia, Evguenia S., "Structural determinants of murine leukemia virus reverse transcriptase that are important for template switching, fidelity, and drug resistance" (2000). Graduate Theses, Dissertations, and Problem Reports. 1239.

https://researchrepository.wvu.edu/etd/1239

This Dissertation is protected by copyright and/or related rights. It has been brought to you by the The Research Repository @ WVU with permission from the rights-holder(s). You are free to use this Dissertation in any way that is permitted by the copyright and related rights legislation that applies to your use. For other uses you must obtain permission from the rights-holder(s) directly, unless additional rights are indicated by a Creative Commons license in the record and/ or on the work itself. This Dissertation has been accepted for inclusion in WVU Graduate Theses, Dissertations, and Problem Reports collection by an authorized administrator of The Research Repository @ WVU.

For more information, please contact researchrepository@mail.wvu.edu. 


\section{STRUCTURAL DETERMINANTS OF MURINE LEUKEMIA VIRUS REVERSE TRANSCRIPTASE THAT ARE IMPORTANT FOR TEMPLATE SWITCHING, FIDELITY, AND DRUG-RESISTANCE}

\section{DISSERTATION}

Submitted to the School of Medicine, Department of Biochemistry of

West Virginia University

In Partial Fulfillment of the Requirements for

The Degree of Doctor of Philosophy

In Biochemistry

By

Evguenia S. Svarovskaia

Morgantown

West Virginia

2000

Committee Members:

Dr. Nyles Charon

Dr. Charles Harris

Dr. Wei-Shau Hu

Dr. Mary Wimmer

Dr. Vinay Pathak, Committee Chair 


\section{ABSTRACT \\ STRUCTURAL DETERMINANTS OF MURINE LEUKEMIA VIRUS REVERSE TRANSCRIPTASE THAT ARE IMPORTANT FOR TEMPLATE SWITCHING, FIDELITY, AND DRUG-RESISTANCE}

\section{Evguenia S. Svarovskaia}

Retroviruses exhibit high mutation rates. High mutation and recombination rates increase variation within a viral population and result in production of drug-resistant mutants and/or mutants that can escape a host immune response. Mutations are introduced into the viral genome by error-prone reverse transcriptase (RT), a virally encoded enzyme that converts single-stranded viral RNA into double-stranded DNA. In the current study we are trying to understand what structural determinants of RTs are important for fidelity, frequency of template switching, and drug-resistance. First, we developed an in vivo assay and performed mutational analysis of murine leukemia virus (MLV) RT to identify structural elements important for template switching. Based on obtained results, we proposed a dynamic copy-choice model in which both the rate of DNA polymerization and the rate of degradation of the RNA template influence the frequency of RT template switching. Second, we employed a previously described in vivo fidelity assay to determine whether a minor groove binding helix of the thumb domain and primer grip of MLV RT are important for in vivo fidelity of reverse transcription. Because the thumb domain of MLV RT has not been crystallized, we utilized homology alignment and molecular modeling to identify the minor groove binding helix of the thumb domain of MLV RT. Mutations in the minor groove binding helix residues R301 and F309 decreased RT fidelity by up to 2.8-fold, suggesting that this region plays an important role in accuracy of DNA synthesis. Finally, we attempted to elucidate a mechanism of drug-resistance to the antiretroviral nucleoside analog $2^{\prime}, 3^{\prime}$-dideoxy-3'-thiacytidine (3TC), an inhibitor of wild-type human immunodeficiency virus type 1 (HIV-1) RT. We tested our hypothesis that a valine residue at the 223 position in YVDD motif of the MLV RT leads to a natural high level of resistance of MLV to 3TC in a manner similar to that proposed for the YVDD mutant of HIV-1 RT. The results indicated that the wild-type, V223M, V223I, V223A, and V223S mutants of MLV RT were all highly resistant to 3TC, suggesting that determinants outside the YVDD motif of MLV RT confer a high level of resistance to $3 \mathrm{TC}$. 


\section{TABLE OF CONTENTS}

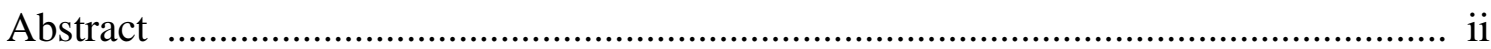

Table of Contents ................................................................................................. iii

List of Tables and Illustrations ....................................................................... V

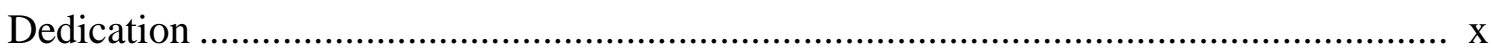

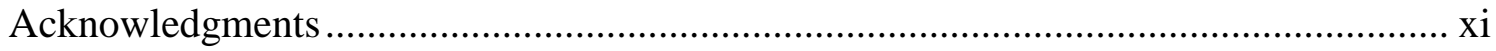

Chapter 1. Introduction and Review of Literature .......................................... 1

Figure Legends and Figures ....................................................... 15

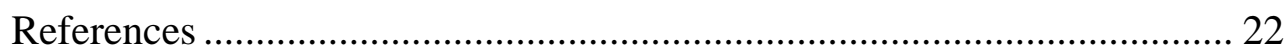

Chapter 2. Structural Determinants of Murine Leukemia Virus Reverse

Transcriptase that Affect the Frequency of Template Switching ............... 34

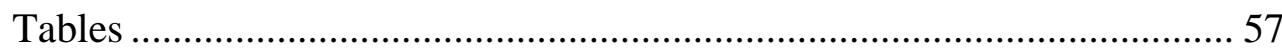

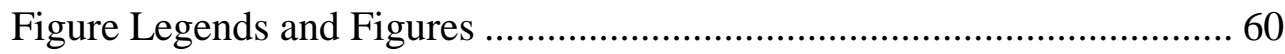

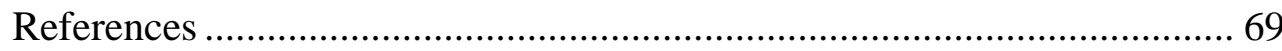

Chapter 3. Identification of Murine Leukemia Virus Minor Groove Binding

Helix of Thumb Domain and Its Role in Fidelity of Reverse

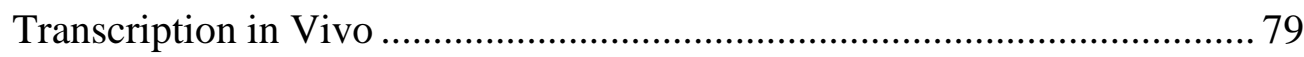

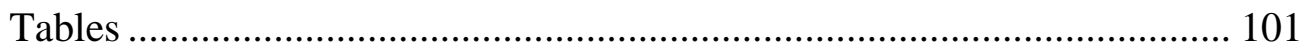

Figure Legends and Figures ........................................................ 104

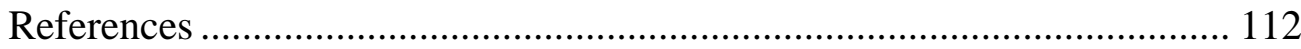

Chapter 4. Wild-Type and YMDD Mutant of Murine Leukemia Virus Reverse

Transcriptase Are Resistant to 2',3'-Dideoxy-3'-thiacytidine ................... 122

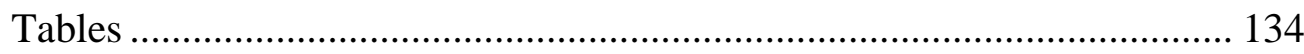


Figure Legends and Figures .......................................................... 136

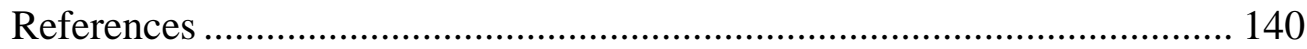

Chapter 5. Discussion and Future Directions .................................................... 150

Figure Legends and Figures ........................................................ 161

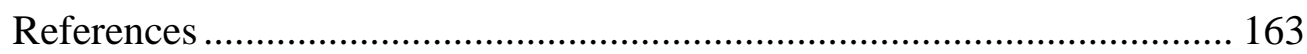

Appendix 1. FACS analysis data for Pol + RNase H double mutants, and F156W and RNase H Mutants in the presence and absence of hydroxyurea.......... 171

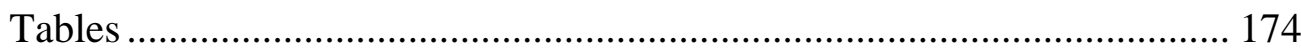

Figure Legends and Figures .......................................................... 176

Appendix 2. Construction of Murine Leukemia Virus Reverse Transcriptase

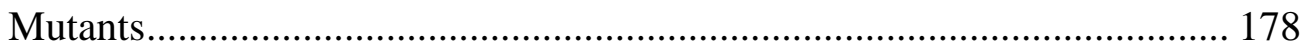

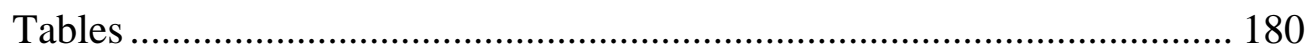

Figure Legends and Figures ........................................................ 182

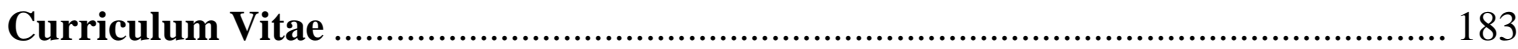




\section{LIST OF TABLES AND ILLUSTRATIONS}

Chapter 1. Introduction and review of literature .................................................. 1

Figure 1: Retroviral replication cycle ........................................... 15

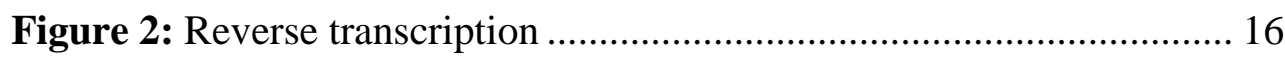

Figure 3: Crystal structure of HIV-1 RT ........................................ 17

A: The p66 subunit consists of the fingers, palm, thumb, connection, and RNase H domain.............................................................. 17

B: Crystal structure of HIV-1 RT bounded to DNA .............................. 17

Figure 4: Model for direct repeat deletions during reverse

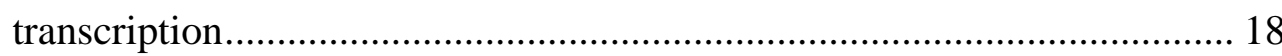

Figure 5: Strategy of construction of ANGIE P cell line ........................ 19

A: Structure of retroviral genome, retroviral vector GA-1 ..................... 19

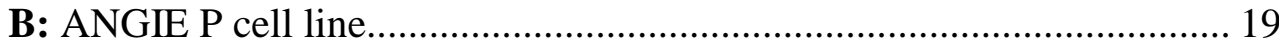

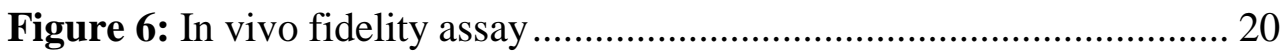

Figure 7: Structure of deoxycytidine and $2^{\prime}, 3^{\prime}$-dideoxy-3'-

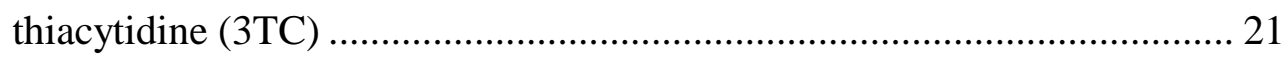

Chapter 2. Structural Determinants of Murine Leukemia Virus Reverse

Transcriptase that Affect the Frequency of Template Switching ............... 34

Table 1: Effect of mutations in MLV RT on the frequency of direct repeat deletion and GFP reconstitution ..................................................5 57

Table 2: Effect of HU treatment on the frequency of direct repeat

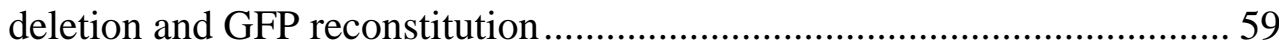


Figure 1: Structures of MLV-based constructs and direct repeat deletion assay to identify structural determinants of MLV RT important for template switching

A: Structures of MLV-based vector pES-GFFP, pLGPS, and pSV-A-

MLV-env 63

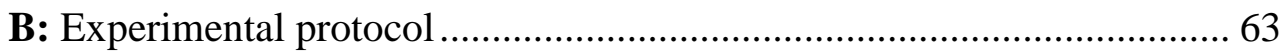

C: FACS analysis of B2-1GFFP and infected D17 target cells .................... 63

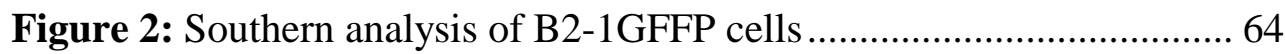

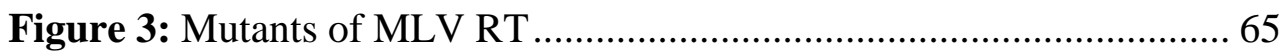

A: The substitution mutations analyzed at each amino acid position .......... 65

B: Primary sequence alignment of the MLV RT and HIV-1 RT

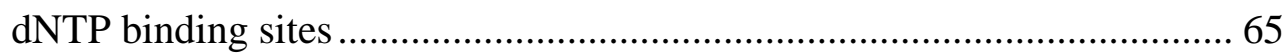

C: Primary sequence alignment of the $\alpha$-helix H of the HIV-1 RT

thumb domain and corresponding sequences in MLV and SNV RTs......... 65

Figure 4: Effect of mutations in the MLV RT dNTP binding site and YXDD motif (A), the $\alpha$-helix $\mathrm{H}$ of the thumb domain $(\mathbf{B})$, and the RNase H domain (C) on the frequency of GFP reconstitution 66 Figure 5: Effect of HU treatment on the frequencies of GFP reconstitution exhibited by the wild-type, V223I mutant, and Y598V mutant of MLV RT 67

Figure 6: Dynamic copy-choice model for RT template switching ........... 68 
Chapter 3. Identification of Murine Leukemia Virus Minor Groove Binding

Helix of Thumb Domain and Its Role in Fidelity of Reverse

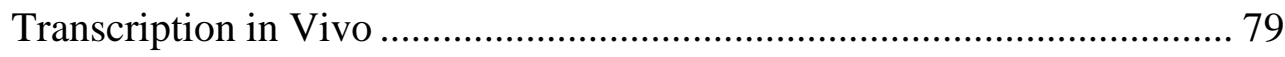

Table 1: Effects of mutations of the MLV RT thumb region on the

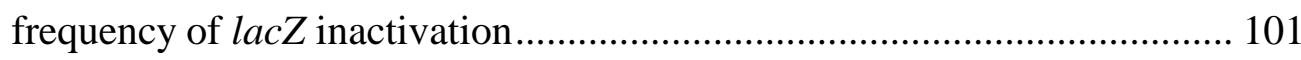

Table 2: Effects of mutations of the primer grip of MLV RT on the frequency of $l a c Z$ inactivation.

Figure 1: Amino acid sequence alignment of primer grip and thumb domain region of retroviral reverse transcriptases

Figure 2: Model of the MLV RT Thumb domain

A: A ribbon representation of the superimposed structure fingers and thumb domain of the MLV RT over the corresponding domain of the HIV-1 RT 108

B: The primer-binding pocket of HIV-1 RT 108

C: Interactions of the minor groove binding helix of MLV RT with the primer 108

Figure 3: Mutational analysis of the minor groove binding helix of

MLV RT 109

A: Protocol for in vivo assay to identify structural determinants of

MLV RT important for fidelity 109

B: Mutants of the primer grip and thumb domain of MLV RT 109

Figure 4: RT activities for virion-associated RTs 110

Figure 5: Decreased processing of mutant viruses..... 111 
Chapter 4. Wild-Type and YMDD Mutant of Murine Leukemia Virus Reverse Transcriptase Are Resistant to $2^{\prime}, 3^{\prime}$-Dideoxy-3'-thiacytidine

Table 1: The effect of 3TC treatment on MLV replication

Figure 1: Structures of MLV-based constructs and protocol used to determine sensitivity to $3 \mathrm{TC}$

A: Structures of the MLV-based vector pGA-1 and the gag-pol expression construct pLGPS 138

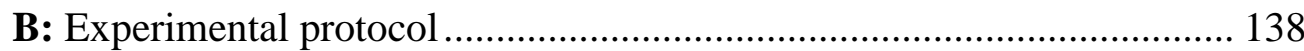

C: Measurement of virus titer ......................................................... 138

Figure 2: Structure of HIV-1-based constructs and activation of 3TC in NIH 3T3, HeLA, 143B, and D17 cell lines

A: Structure of the HIV-1-based luciferase-expression vector pNLuc, which contains both LTRs, other cis-acting elements required for viral replication, and the gag-pol gene of HIV-1

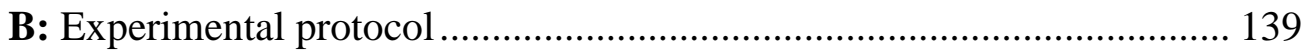

C: Measurement of chemiluminescence ................................................... 139

Chapter 5. Discussion and Future Directions ...................................................... 150

Figure 1: A schematic representation of in vivo assay to select for RT with a high fidelity 161

Figure 2: Template switching model for minus-strand and plusstrand transfers 162

Appendix 1. FACS analysis data for Pol + RNase H double mutants, and F156W and RNase $\mathrm{H}$ Mutants in the presence and absence of hydroxyurea 171 
Table 1: Effect of mutations in MLV RT on the frequency of direct repeat deletion and GFP reconstitution ................................................ 174

Table 2: Effect of HU treatment on the frequency of direct repeat

deletion and GFP reconstitution 175

Figure 1: Effect of mutations in the MLV RT pol domain and

RNase $\mathrm{H}$ domain on the frequency of GFP reconstitution 176

Figure 2: Effect of HU treatment on the frequencies of GFP reconstitution exhibited by the wild-type, and S526A, R657A, and F156W mutants of MLV RT 177

Appendix 2. Construction of Murine Leukemia Virus Reverse Transcriptase

Mutants 178

Table 1: Sequences of the primers utilized in generation of MLV

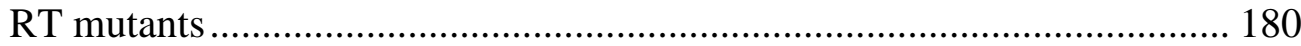

Table 2: Codons of the generated mutants ............................................ 181

Figure 1: PCR-based mutagenesis protocol .......................................... 182 


\section{T his D issertation is $D$ edicated to $M$ y $F$ amily}




\section{ACKNOWLEDGMENTS}

There are a number of people, who, in one way or another, contributed to the completion of this dissertation. First and foremost among them is my research advisor Dr. Vinay K. Pathak. His scientific integrity, hard work, and dedication beamed my way through the complex labyrinths of my research.

I am also grateful to my research committee, Drs. Nyles Charon, Charles Harris, Wei-Shau $\mathrm{Hu}$, and Mary Wimmer, for their advice and constructive criticisms of this work. I especially would like to thank Dr. Wei-Shau Hu, who lent me her invaluable help when I most needed it.

I would like to extend my appreciation to all members of Dr. Pathak's and Dr. Hu's laboratories for fun time, friendly atmosphere, and for helping me with any difficulties that arose during my research. Especially I would like to thank Lou Halvas, Carey Hwang, and Krista Delviks, who collaborated with me on several projects.

I also would like to like to acknowledge the financial support in the form of teaching assistantship for the first year of my study by the WVU Department of Biochemistry.

Finally, I would express my sincere gratitude to my family, my husband Sergei, and my daughters, Katya and Michelle, for unending patience, understanding, and love, which made possible completion of this work; my parents Olga Sergeevna and Sergei Ivanovich Favorski, and my brother Vladimir Sergeevich Favorski, who always encouraged me to pursue altitudes of knowledge and never give up. 


\section{CHAPTER 1}

INTRODUCTION AND REVIEW OF LITERATURE 
Approximately 34 million people worldwide are currently infected with human immunodeficiency virus type-1 (HIV-1) (1). The vast majority of these individuals are destined to die of acquired immunodeficiency syndrome (AIDS) unless truly effective therapy can be developed (74). For them, as well as many more who will become infected within the next few years, any future vaccine will come too late, and effective therapy must be developed to combat existing infection. The recent application of therapies using combinations of antiviral drugs has shown that virus growth in infected people can be brought to an undetectable level and, in many individuals, provide considerable and long-lasting improvement in their condition (6). These therapies have helped large numbers of people live relatively normal lives despite their HIV-1 infection; however, these drugs do not provide a long-term solution. The problem is in the development of resistant viral variants due to the appearance of specific mutations (6). In an effort to avoid resistance, drugs have to be given at high toxic doses and in expensive combinations. Even then, the therapy often fails, and resistant virus appears. There is, therefore, a desperate need to understand how the virus develops resistance to drugs, and to use this understanding to develop more effective strategies for treating HIV-1 infection.

Retroviral life cycle. HIV-1 belongs to retroviruses, a family of RNA viruses that replicate via a DNA intermediate (15). A schematic representation of the retroviral replication cycle is shown in Fig. $1(15,52,69)$. Initiation of retrovirus replication involves a highly specific interaction between a virion envelope proteins with a cellsurface receptor. The virus envelope and the cell membrane fuse to release the viral core into the cytoplasm. After entry of the core into the cytoplasm, the RNA genome is 
reverse transcribed into double-stranded DNA. Integration of the viral DNA into random sites in the cell chromosome forms the provirus. Viral RNA is synthesized by cellular RNA polymerase II which uses the integrated provirus as a template. Synthesis of viral proteins is followed by formation of viral particles at the plasma membrane. The viral RNAs, which have a special encapsidation signal (named the $\psi$-region), can be packed into virus. The newly made virion can then repeat the cycle by infecting other cells. Mutations could be possibly introduced into retroviral genome at three different steps of viral replication. First, the reverse transcriptase (RT), a virally encoded enzyme, can potentially introduce mutations into the viral genome during the process of reverse transcription, which involves both RNA- and DNA-dependent DNA syntheses. The mutation rate of RTs was estimated to be around $10^{-5}$ mutations per base pair per replication cycle $(41,42,51,53-55,58)$. Second, cellular DNA polymerases replicate the provirus in each cell cycle and also could mutate proviral DNA. However, cellular DNA polymerases possess proofreading mechanisms and as a result cellular DNA replication has very low mutation rates $\left(10^{-9}\right.$ to $10^{-11}$ substitutions per base pair per cell division). Therefore, it is unlikely that cellular DNA polymerases are the major contributors to mutations introduced in the retroviral genome.(25). Finally, RNA polymerase II synthesizes the RNA genome of the next generation of virions. Fidelity of RNA polymerase II was recently measured in vitro to be between $10^{-3}$ and $10^{-5}$ misincorporations / bp / cycle (46). These data provide a large range for the fidelity of RNA polymerase II. More recent studies indicate that this measurement was an overestimate of the RNA polymerase II mutation rate. Because the mutation rates of RT and RNA polymerase are comparable it is not clear which of the enzymes contributes to 
retroviral mutation rate the most. To sort out these possibilities it has been recently shown that at least one third of the retroviral mutations (32\%) are introduced into a retroviral genome during plus-strand synthesis and approximately two third of mutations (68\%) are introduced during minus-strand synthesis and RNA transcription (37). These data indicate that the RNA polymerase II mutation rate cannot be grater than $2 / 3$ of the retroviral mutation rates $\left(1 \times 10^{-5} / \mathrm{bp} /\right.$ replication cycle). Mutations that occur during DNA dependent DNA synthesis result in mismatches. Recent studies have indicated that mismatches involving large loops can be efficiently repaired by the host repair system affect the overall mutation rate (11).

Reverse transcription. Reverse transcription is a process in which the viral RNA is copied into a double-stranded DNA (Fig. 2) $(24,52)$. In the initial step of reverse transcription, a tRNA primer associates with RT at the primer-binding site (PBS) near the 5' end of viral RNA, is utilized to synthesize the short piece of DNA. This short piece DNA is called the minus-strand strong-stop DNA. Along with synthesis, the RNase $\mathrm{H}$ activity of RT degrades the RNA template in RNA-DNA hybrids approximately 18 bases behind the 3'OH of the primer. Once the synthesis of the minus-strand strong-stop DNA is accomplished, the minus-strand strong-stop DNA is transferred to the 3' end of the viral RNA using complementarity of the terminal repeat (R) regions. Consequently, the minus-strand strong-stop DNA is extended to form the minus-strand DNA. The minusstrand DNA is also accompanied by RNase $\mathrm{H}$ degradation of the RNA template. The RNA sequence named polypurine tract (PPT) is resistant to complete RNase $\mathrm{H}$ degradation and forms a specific primer for initiation of plus-strand synthesis. The plusstrand DNA synthesis is initiated by synthesizing of a piece of DNA called plus-strand 
strong-stop DNA. The plus-strand strong-stop DNA ends with a PBS sequence as a result of copying 18 bases of tRNA. It has been proposed that copying of the tRNA ends at the first modified base of the tRNA. The plus-strand strong-stop DNA is transferred to the 5' end of the viral DNA by complementation at the PBS. DNA synthesis continues in both directions to complete the synthesis of viral DNA. As a result of reverse transcription, the viral double-stranded DNA has long terminal repeats (LTRs) at both ends. LTRs consist of U3 (unique 3'), R, and U5 (unique 5') regions. By using the LTRs, the double-stranded DNA integrates into the host chromosome to form a provirus. Crystal structure of RTs. Several crystal structures of HIV-1 RT have been reported, including co-crystals with non-nucleoside inhibitors, DNA, and a ternary complex with bound DNA and a substrate $\operatorname{dTTP}(17,31,38,65)$. The enzyme is a heterodimer composed of 66 and $51 \mathrm{kD}$ subunits (Fig. 3A). The structure of p66 subunit resembles a right hand and is referred to as the fingers, palm, connection, thumb, and the RNase $\mathrm{H}$ domains $(17,31,38,65)$. The thumb domain consists of a bundle of $\alpha$-helixes. The p51 subunit is composed of four domains referred to as fingers, thumb, palm, and connection. Only the p66 subunit possesses the polymerization and RNase $\mathrm{H}$ activities (38). The highly conserved YMDD motif constitutes the active site of the enzyme (32). The $\beta 12$ $\beta 13$ hairpin is thought to act as the primer grip and is important for positioning the 3' $\mathrm{OH}$ of the primer into the active site of the RT. The highly conserved LPQG box has been proposed to form hydrogen bonds with the incoming dNTP and the template (61). Recently, a crystal structure of a ternary complex of HIV-1 RT with bound DNA and a substrate dTTP (Fig. 3B) (31) was solved. This structure revealed detailed interactions of RT with template-primer complex as well as dTTP substrate. For example, all amino 
acids that form dNTP-binding pocket were identified. Amino acids K65, R72, D113, A114, Y115, and Q151 were shown to directly interact with the incoming dNTP. It was also shown that the $\alpha$-helix $\mathrm{H}$ of the thumb domain fits into the minor groove of the template-primer complex and form interactions with the second, third, and forth bases upstream of the 3' end of the primer. More excitingly, using these crystal structure data it became possible to measure any distances within this ternary complex.

The crystal structure of an MLV RT fragment (amino acids 24 - 274) consisting of the fingers and palm domains has also been determined (23). The MLV RT is a $75 \mathrm{kD}$ monomer that has been proposed to form a homodimer in the presence of substrate (68). The fingers and palm domains of this crystallized fragment fold in a very similar manner as the fingers and palm domains of HIV-1 RT. Even though HIV-1 and MLV RTs share only $25 \%$ sequence identity, their overall folds in the fingers and palm domains are incredibly similar $(64,73)$. Recently, crystal structures of the same fragment containing the fingers and palm domains of MLV RT were obtained in the presence of DNA (47). These structures showed a different form of DNA binding in comparison to HIV-1 RT. These differences are likely to be explained by the absence of the thumb domain in the crystallized fragment of MLV RT, which was shown previously to be important for proper binding of the template-primer complex.

High frequency of large direct repeat deletion by RTs. Previous studies have shown that directly repeated sequences found in retroviral vectors and genomes tend to be highly unstable $(34,49)$ and are believed to be deleted through the normal process of reverse transcription. For example, deletions studied in SNV have shown that direct repeats of 1333,788 , and 383 bp were deleted at frequencies of $93 \%, 85 \%$, and $40 \%$ respectively 
(34). The template misalignment model has been proposed to explain the phenomena of direct repeat deletion in retroviruses (34). Recent data has indicated that direct repeat deletions occur nearly at the same rate during RNA-dependent and DNA-dependent DNA synthesis (49).

A modified template misalignment model of direct repeat deletion is shown in Fig. 4 (16). The model proposed that base-pairing between newly synthesized DNA sequences $3^{\prime}$ to the RT with complementary sequences of the template increases the probability of RT switching templates. As RT copies the 3' direct repeat, RNase H degrades the RNA template $3^{\prime}$ to the RT. Subsecuently, hydrogen bonding occurs between the newly synthesized single-stranded DNA and complementary sequences in the $5^{\prime}$ copy of the direct repeat. The conformational rearrangements that permit this hydrogen bonding are depicted as a loop in the template RNA in Fig. 4. The hydrogenbonding interactions 3' to the RT bring the homologous acceptor template in close proximity to the RT, subsequently leading to a template switch.

The frequency of direct repeat deletions may be used to estimate the processivity of RTs. Lower processivity of RT results in more frequent dissociation from the template and consequently higher frequency of direct repeat deletions. It is likely that mutations in RT that affect processivity affect the frequency of template switching; however, structural determinants of RTs that are important for direct repeat deletions and template switching remain to be identified. The direct repeat deletion assay in vivo can provide a powerful model to identify these determinants.

Assays for measurement of the fidelity of RTs. The fidelity of RTs can be measured by utilizing purified RT with a template-primer and appropriate dNTPs. This kind of 
assay would be considered an in vitro assay. These reactions are usually performed on templates that are different from the templates used by RT during viral reverse transcription and at different reaction conditions such as dNTP concentration, $\mathrm{Mg}^{2+}$ concentration, and $\mathrm{pH}(13,19,43)$. Additionally, the fidelity of DNA polymerases may be altered by purification procedures trough proteolytic loss of the proofreading domain $(39,56)$. As a result, RT fidelity measured in an in vitro assay may be significantly different from RT fidelity during reverse transcription. The estimation of the RT fidelity of the replication-competent virus in vivo was difficult because the number of replication cycles were not known. The approach of the packaging cell line, which allows production of virus capable of infecting target cells only once, has been developed. The one cycle replication assays can be used to measure the rate of mutations introduced by the RT in a reporter gene during reverse transcription in vivo $(41,42,53,54)$. Comparison of HIV-1 in vitro and in vivo mutation rates indicated that the in vitro error rates were 16-20 fold higher than the in vivo mutation rate (41).

Recently, an in vivo fidelity assay was developed to study structural determinants that are important for fidelity (27). First, ANGIE P encapcidating cell line was developed. To develop the ANGIE P, a MLV cell line retroviral genome was divided into three constructs: pLGPS that expressed gag-pol retroviral proteins; pA-MLVenv that expressed the amphotropic MLV envelope; and retroviral vector pGA-1 that encoded all cis-acting elements necessary for packaging of viral RNA and completion of reverse transcription (Fig. 5). In addition, the retroviral vector pGA-1 encoded the $\beta$ galactosidase reporter gene and neomycin resistance gene. The ANGIE P cell line was developed by stable transfection of D17 cells with pA-MLVenv and retroviral vector 
pGA-1. Upon transfection of wild type or RT mutant pLGPS construct into ANGIE P cells, the cells produced virus that contain wild type or mutant RT and GA-1 viral RNA (Fig. 6). The collected virus was used to infect target cells. During reverse transcription mutations were introduced into $\beta$-galactosidase reporter gene by wild type or mutant RT and visualized by staining the infected cells with $\mathrm{X}-\mathrm{Gal}$, a color substrate for functional $\beta$-galoctosidase. Blue colonies contain no inactivating mutations in $\beta$-galoctosidase, where as white colonies contain an inactivating mutation in $\beta$-galactosidase. Comparison of the frequencies of the white and blue colonies for wild type RT and mutant RT provided an estimation of the effect of a particular mutation on RT fidelity.

Structural determinants of the RT that are important for fidelity. The genetic variation in retroviral populations is generated by their high rates of mutations and recombination $(14,29,30,41,42,51,53-55,57,70)$. There are two possible explanations for the low fidelity of RTs. First, low processivity of RT was likely to be selected by evolution because it is essential for the process of reverse transcription. The RT needs to be easily dissociated from the template in order to accomplish the minusstrand and plus-strand transfer events that are necessary for completion of reverse transcription (70). Second, it was postulated that RT lacks proofreading activity. However, currently this statement is being questioned. It was found that HIV-1 develops resistance to the 3'-azido-3'-deoxythymidine (AZT), a nucleoside analog that inhibits reverse transcription by termination DNA polymerization reaction because it lacks the 3' $\mathrm{OH}(3,4)$. Resistance to AZT is associated with the ability of the drug-resistant mutant of the HIV-1 RT to remove the incorporated AZT from the 3' end of the synthesized 
DNA. This activity is mainly associated with a drug-resistant RT mutant, but wild type HIV-1 RT is also capable of performing this reaction, although less efficiently $(3,4)$.

Structural determinants of RT that are important for accuracy of the enzyme are currently unknown. Based on the crystal structure of the RT and experimental evidence it was proposed that the YXDD box, the LPQG box, the dNTP binding site, the $\alpha$-helix H of the thumb domain, and the RNase H domain may play a role in the fidelity of RT. Each of these domains are briefly discussed bellow.

YXDD box: Experimental evidence supports that the YXDD box is important for fidelity. Mismatch extension assays performed in vitro showed that the M184V mutation of the YXDD box increases fidelity of the HIV-1 RT (72). The same result was reproduced by another research group which demonstrated that the order of accuracy of 184 variants in vitro is Ile $>$ Val $>$ Met (50). However, in vivo studies did not show a significant change in fidelity for M184V (48). In addition, an M13 phage-based forward mutation assay indicated that $\mathrm{M} 184 \mathrm{~V}$ and $\mathrm{E} 89 \mathrm{G}$ mutants, as well as double mutant (M184V/E89G), do not have a significant change in fidelity as shown in vitro (18). This result is in agreement with in vivo data from our laboratory, which indicates that the difference in fidelity between M and V variants of YVDD box of MLV RT is not more than two fold (27). Additionally, other mutants showed that the order of fidelity in vitro is M184L>Y183F>WT HIV-1 RT (5). Other studies of mutants of YXDD box have indicated M184I and M184V display a slight reduction in processivity and the M184L and M184A mutants show considerable reduction in processivity (12).

Thumb domain: The amino acids of the $\alpha$-helix H of HIV-1 RT were mutated one by one to alanines and fidelity and template-primer binding of constructed mutants were 
tested in vitro. The results showed that G262A and W266A possess low fidelity and were demonstrated to be important for primer-template binding (7). Another in vitro study confirmed this result exhibiting lower processivity and lower fidelity of the G262A and W266A mutants by comparison with wild type HIV-1 RT (8).

dNTP binding site: The F155 of the dNTP binding site of MLV RT was proposed to play a role in discrimination between incoming dNTPs $(20,44,45,61)$. Fifteen mutants at position Y115 showed a decreased fidelity in vitro indicating the importance of the dNTP binding site for accuracy of DNA synthesis $(44,45)$. The position 155 is a part of the dNTP binding site of MLV and is analogous to Y115 in HIV-1 RT. Recently, the role of this and other residues of MLV RT were studied in an in vivo fidelity assay. The results indicated that most of the mutants of the dNTP-binding site of MLV RT resulted in noninfectious virus. The mutants with a detectible titer deceased fidelity up to 2-fold (28).

RNase H: RNase H mutations affect RT processivity and interactions of the RT with its template, which may alter the fidelity of RT (68). Additionally, the RNase H domain is believed to play an important role in template-switching events, which result in deletions and insertions during reverse transcription $(22,34,40)$. The S526A, R657S, and Y598V mutations of MLV RT were tested in an vivo fidelity assay in our laboratory. The S526A and R657S RNase H mutants showed approximately 1.4- and 1.2-fold increases in mutant frequency, respectively (27).

Other regions: Some other specific residues of HIV-1 RT have been shown to affect fidelity in vitro. E89G, a nucleoside analog-resistant variant of HIV-1 RT, exhibited 2-17 fold increase in fidelity in vitro (18). This result was confirmed by another in vitro study 
indicating the order of fidelity of drug-resistant variants to be E89G>L74V > WT HIV RT (59). However, the HIV-2 mutants did not show a significant increase in fidelity: the E89G mutant showed some enhanced fidelity, but L74V, in contrast to HIV-1, has showed a fidelity similar to that of wild type HIV-2 (67). Additionally, the importance of the F160, D76, and L74 of HIV-1 RT for fidelity was studied. The F160W mutant displayed a slightly higher fidelity, but it was shown only in vitro at a high dNTP concentrations (26). The D76V resulted in a 14-fold increase in fidelity in vitro (36). The L74V resulted in a 3.5-fold higher fidelity in vitro $(33,63)$.

Mechanisms of resistance to 3TC. Two viral enzymes, RT and protease, are the most common targets for the anti-retroviral drugs. Drug treatment invariably results in the selection of drug-resistant variants that are already present in the viral population. There are two classes of RT inhibitors: nucleoside analogs and non-nucleoside inhibitors. Nucleoside analog RT inhibitors lack the $3^{\prime} \mathrm{OH}$ of the ribose ring. In the cytoplasm of the target cell, nucleoside analogs are converted into nucleoside-analog triphosphates which are consequently incorporated into viral DNA by HIV-1 RT and, as a result, cause the termination of DNA polymerization. Treatment of HIV-1 infected patients with $2^{\prime}, 3^{\prime}-$ dideoxy-3'-thiacytidine (3TC), a nucleoside analog inhibitor of HIV-1 RT, results in the selection of drug-resistant variants with changes at position 184 of the YMDD motif (9, $21,35,62,71)$. Usually the variant M184I appears first and then is replaced by the M184V variant (10). Viruses carrying either of these mutations are very resistant for 3TC in vivo and in vitro. Structural analyses have provided models to explain the high level of resistance of the M184I and M184V mutants for 3TC. The combination of introducing a sulfur atom into the ribose ring and choosing the opposite orientation causes 
the portion of the modified ribose ring that carries the sulfur atom to project farther than the normal ribose ring, thus creating an opportunity for steric hindrance (structure of 3TC is shown in Fig. 7) (60). The inhibition of the wild-type enzyme by $3 \mathrm{TC}$ suggests that 3TC easily incorporates without significant steric hindrance with methionine at position 184. On the other hand, based on the molecular modeling of 3TC-resistant variants of HIV-1 RT, a $\beta$-branched amino acid, such as I or V, at position 184 would interfere with the ability of 3TC triphosphate to bind in the correct orientation at the RT active site (60). This hypothesis also might explain the natural resistance to $3 \mathrm{TC}$ of variants of feline immunodeficiency virus that have another $\beta$-branched amino acid residue (threonine) at a position equivalent to 184 of HIV-1 RT (66) and the resistance of hepatitis B virus MLV RT (2) with either valine or isoleucine at the position equivalent to 184 of HIV-1 RT. Overview of thesis. The purpose of the current work was to elucidate structural determinants that are responsible for the high frequency of template switching and the low fidelity of RT as well as to understand the mechanisms of development of drugresistance by RTs. In chapter 2, we discuss how mutations of the YXDD motif, the dNTP-binding site, the thumb domain, and the RNase H domain of MLV RT affect the frequency of template switching during reverse transcription in vivo. Based on the results, we proposed a dynamic copy-choice model in which both the rate of DNA polymerization and the rate of degradation of the RNA template influence the frequency of RT template switching. The third chapter is devoted to understanding whether a minor groove binding helix of the thumb domain and primer grip of MLV RT are important for in vivo fidelity of reverse transcription. Homology alignment and molecular modeling was utilized to identify the minor groove binding helix of MLV RT thumb domain. 
Finally in chapter 4, we attempt to dissect the mechanism of drug-resistance to 3TC. We test a hypothesis that the presence of a valine residue at 223 position in YVDD motif of the MLV RT leads to a natural high level of resistance of MLV RT to 3TC in a manner similar to that proposed for YVDD mutant of HIV-1 RT. In chapter 5 we discuss how these studies contributed to the overall knowledge of retroviruses and future experiments that could be done to further investigate mechanisms of generating high genetic variation in retroviral populations. 


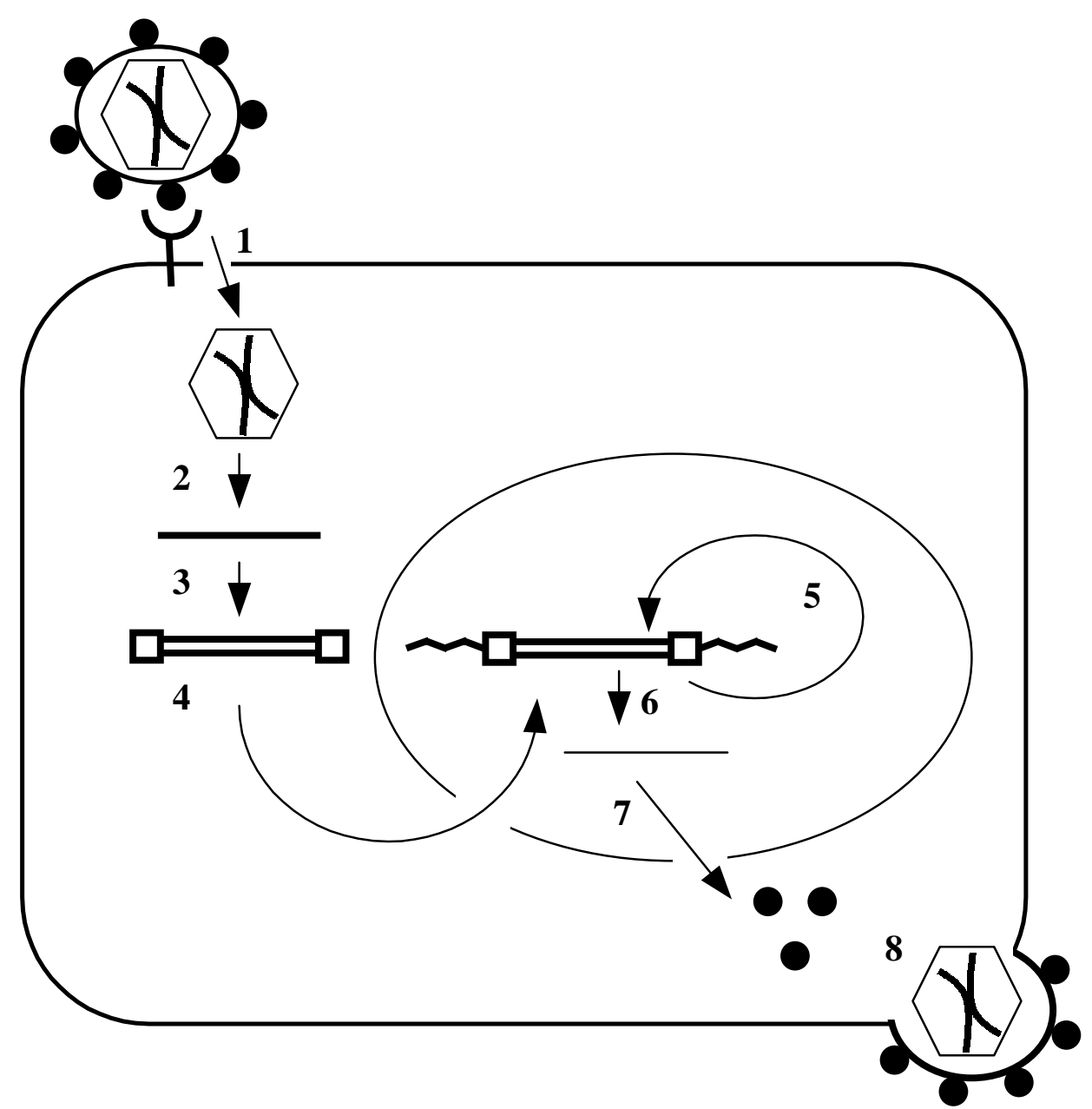

Figure 1. Retroviral replication cycle. Steps in replication designated by numbers as follows: entry (1), minus-strand synthesis (2), plus-strand synthesis (3), integration (4), replication as a provirus by cellular polymerases (not required) (5), transcription (6), translation (7), assembly, packaging, and budding (8). 


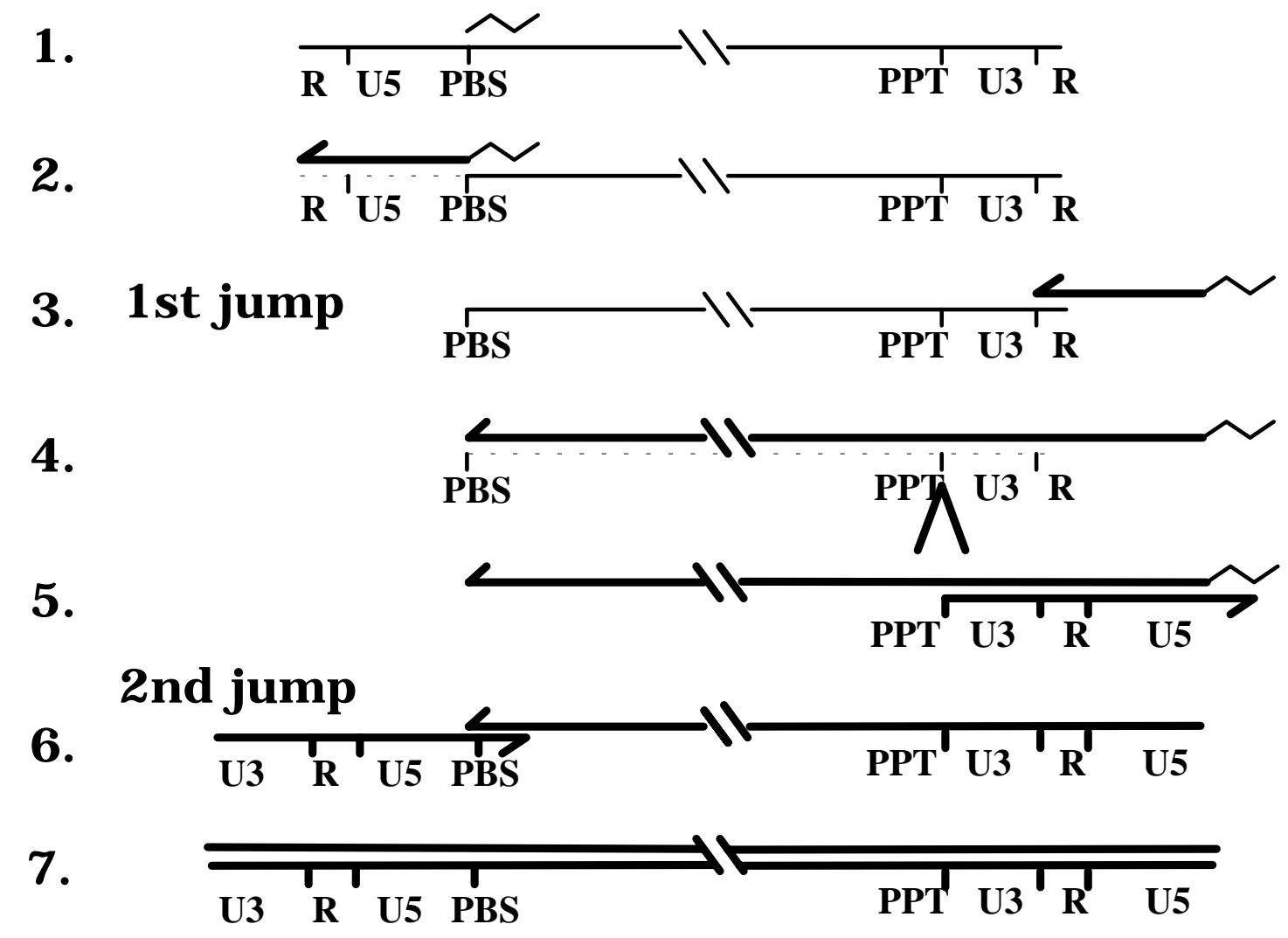

Figure 2. Reverse transcription. Taken from ref. 52. Thin line represents RNA, bold line represents DNA, and dashed lines represent RNase $\mathrm{H}$ degradation. A zigzag line represents tRNA primer. 
A.

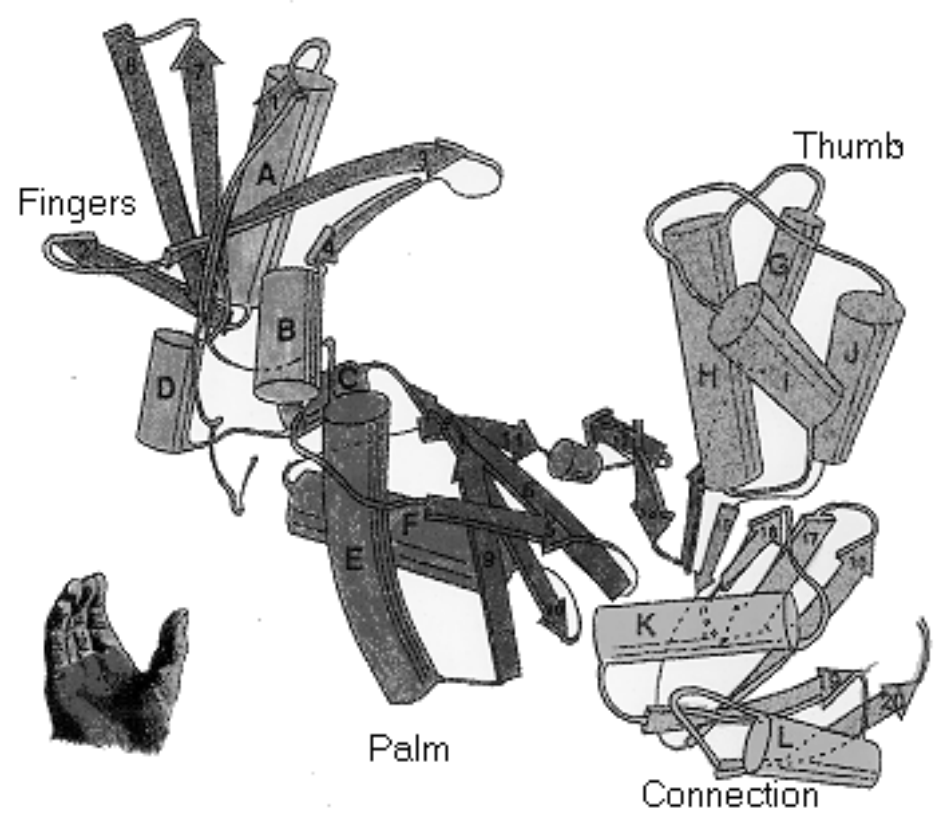

B.

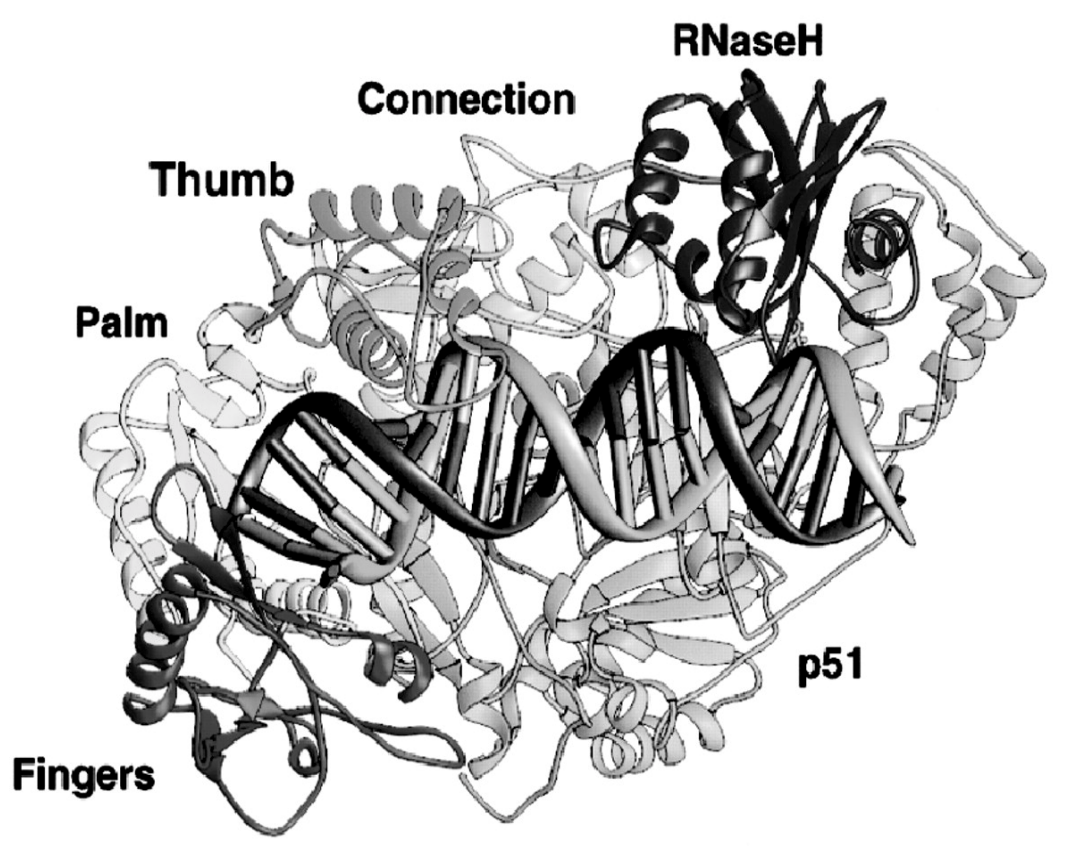

Figure 3. Crystal structure of HIV-1 RT. (A) The p66 subunit consists of the fingers, palm, thumb, connection, and RNase H domain. Figure was modified from ref. 32. (B) Crystal structure of HIV-1 RT bounded to DNA. Figure was modified from ref. 30. 

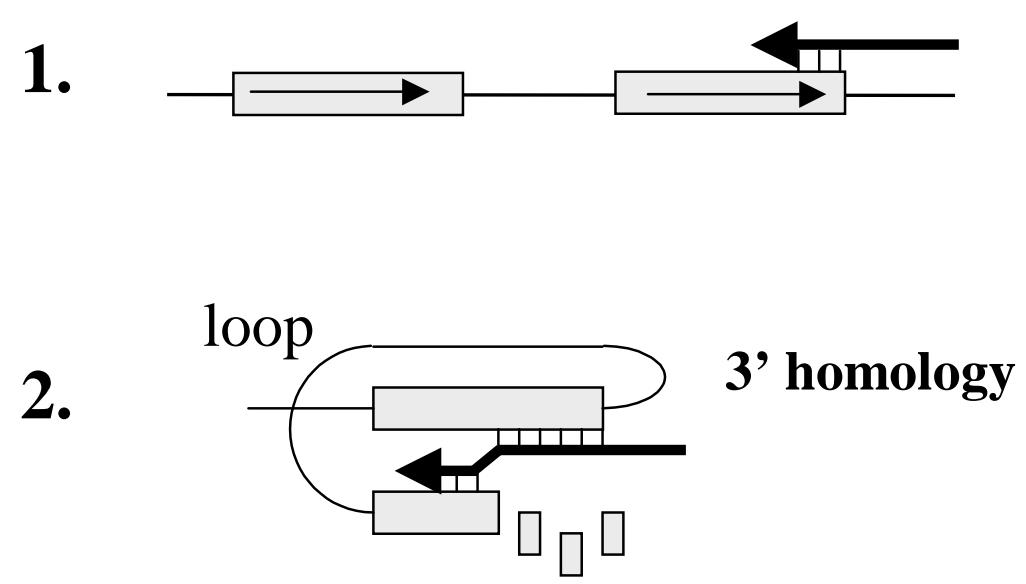

3.
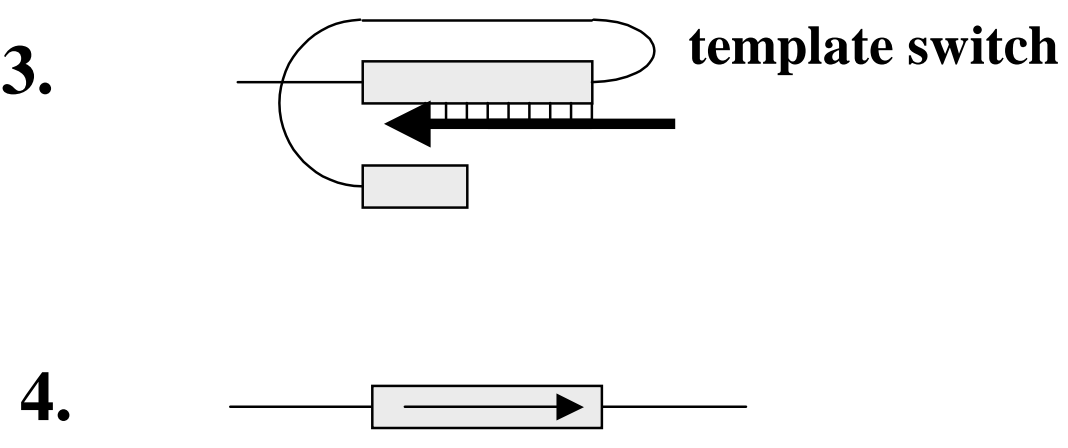

Figure 4. Model for direct repeat deletions during reverse transcription. Shaded boxes represent direct repeats. Horizontal arrows represent nascent DNA. Dashed lines represent degraded RNA by the RNase H domain. Hydrogen bonds between the RNA template and nascent DNA are designated by vertical marks. (1) RT copies the $3^{\prime}$ direct repeat, (2) RNase H degrades the RNA template $3^{\prime}$ to the RT. Subsecuently, hydrogen bonding occurs between the newly synthesized single-stranded DNA and complementary sequences in the $5^{\prime}$ copy of the direct repeat. The conformational rearrangements that permit this hydrogen bonding are depicted as a loop. (3) The hydrogen-bonding interactions 3' to the RT bring the homologous acceptor template in close proximity to the RT, subsequently leading to a template switch. (4) Final product contains only one copy of direct repeats. 

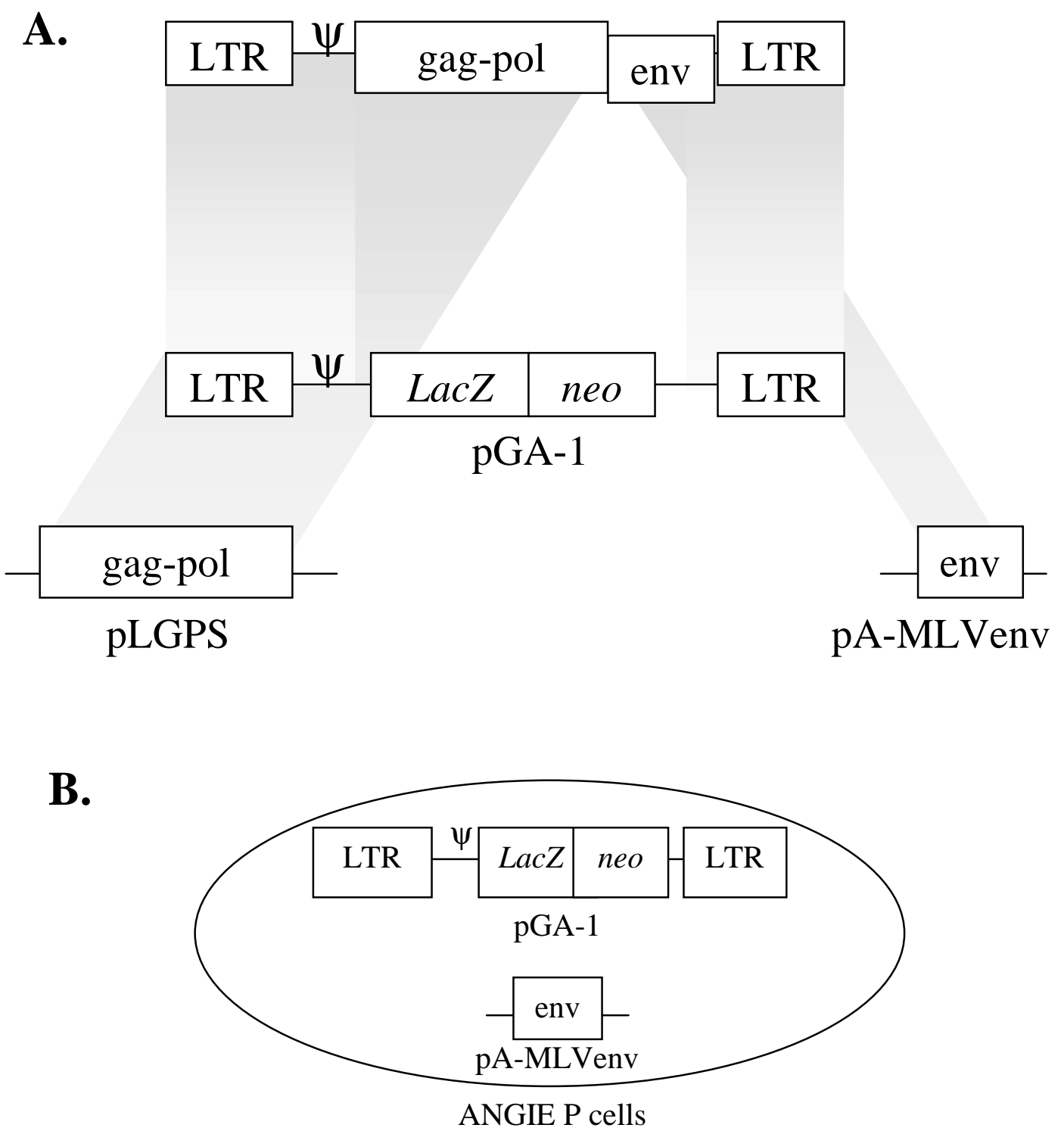

Figure 5. Strategy of construction of ANGIE P cell line. (A) Structure of retroviral genome, retroviral vector GA-1. LTR-long terminal repeats; LacZ - $\beta$-galoctosidase gene; neo - neomycin resistance gene; pLGPS - gag-pol expressing construct; pA-MLVenv amphotropic MLV envelope expressing construct; $\psi$ - packaging signal. (B) ANGIE P cell line. 


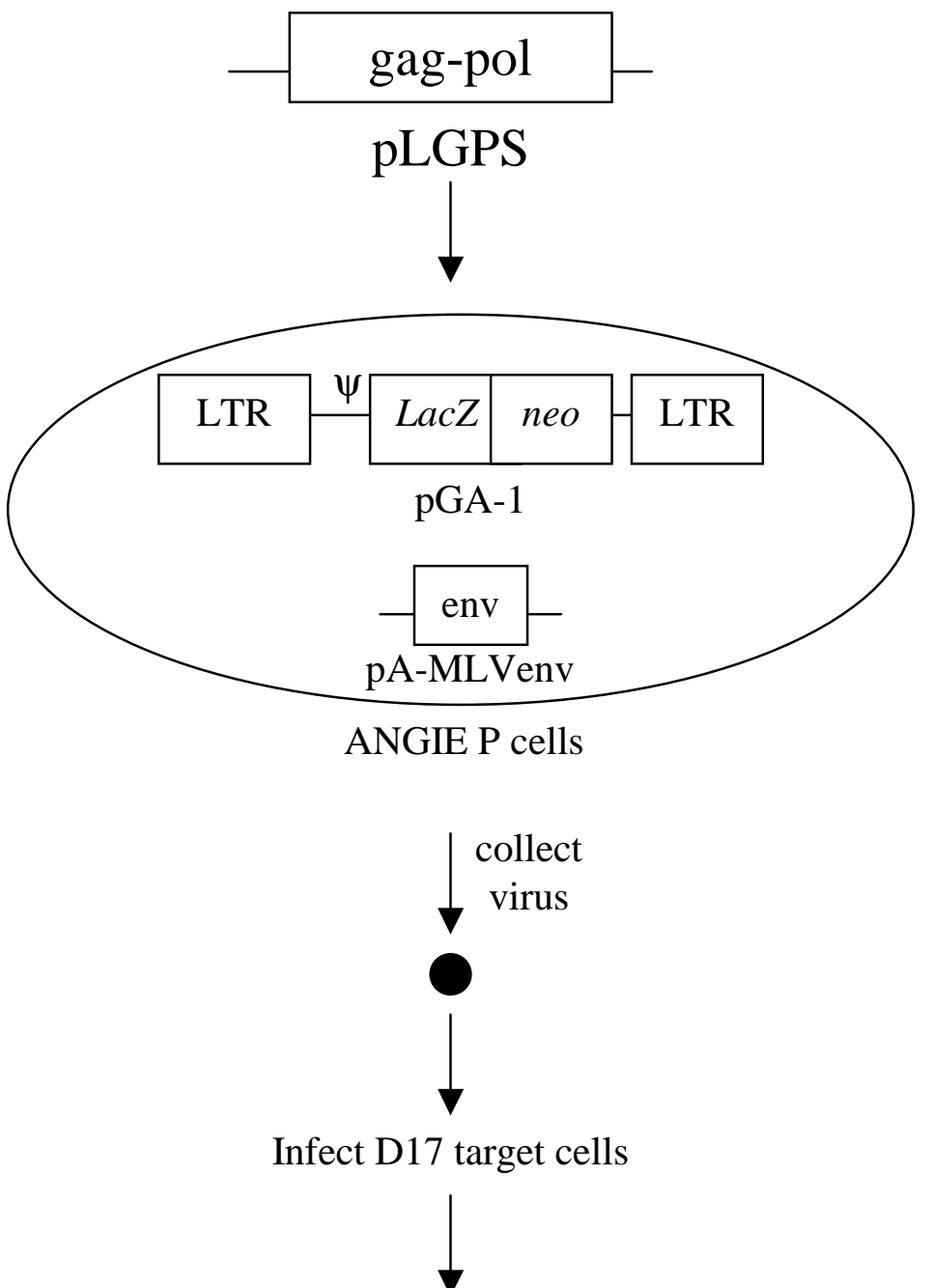

Select for neomycin resistance

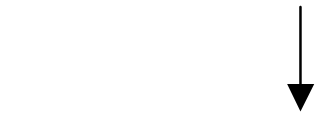

$\mathrm{X}-\mathrm{Gal}$ staining of resistant colonies

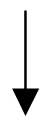

Determine the frequency of LacZ inactivation

Figure 6. In vivo fidelity assay. All abbreviations are as described for Fig. 5. 

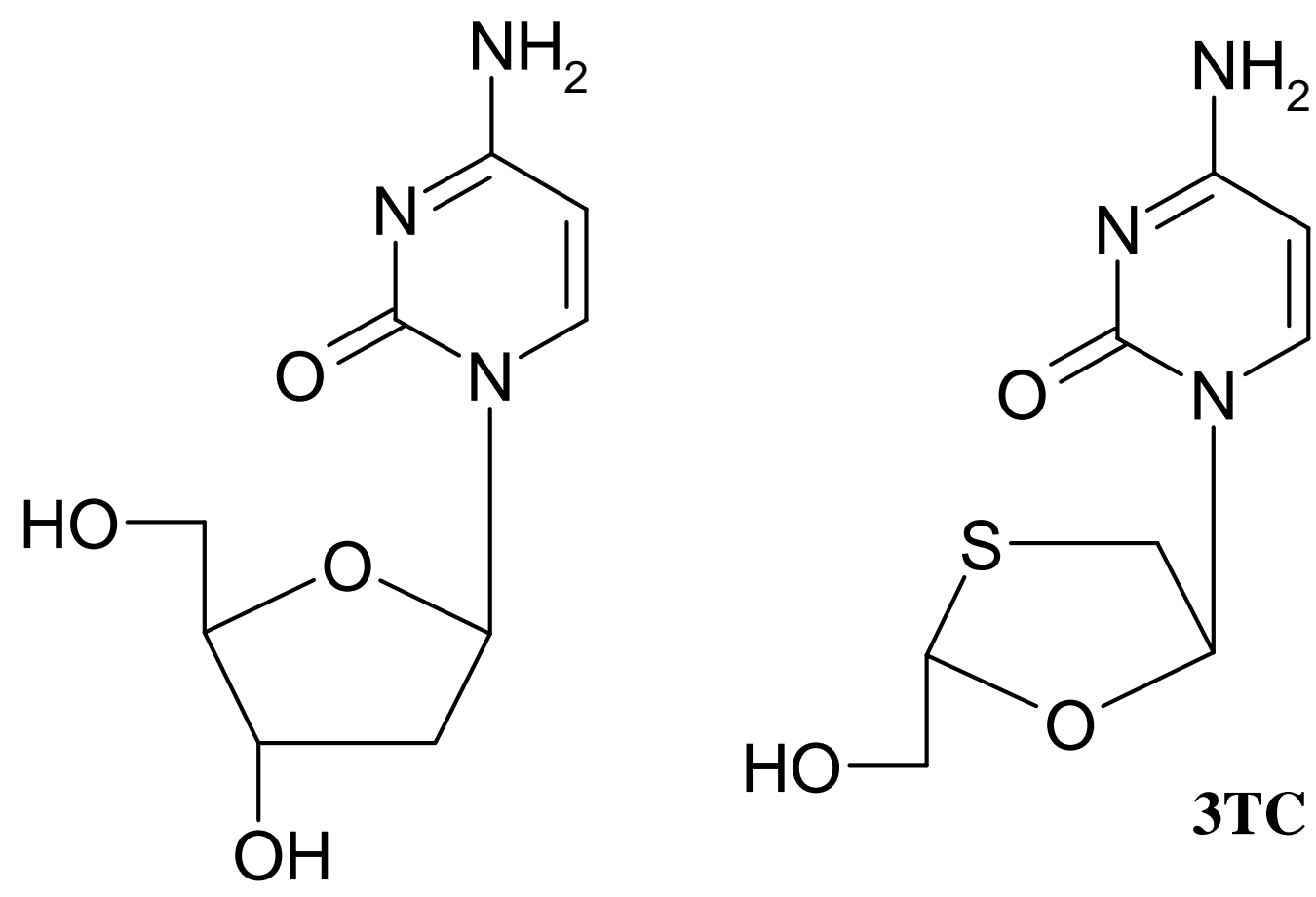

Figure 7. Structure of deoxycytidine and $2^{\prime}, 3^{\prime}$-dideoxy-3'-thiacytidine (3TC). 


\section{REFERENCES}

1. UNAIDS. AIDS epidemic update. December 1999. www.unaids.org.

2. Allen, M. I., M. Deslauriers, C. W. Andrews, G. A. Tipples, K. A. Walters, D. L. Tyrrell, N. Brown, and L. D. Condreay. 1998. Identification and characterization of mutations in hepatitis B virus resistant to lamivudine. Lamivudine Clinical Investigation Group. Hepatology 27:1670-1677.

3. Arion, D., N. Kaushik, S. McCormick, G. Borkow, and M. A. Parniak. 1998. Phenotypic mechanism of HIV-1 resistance to 3'-azido-3'-deoxythymidine (AZT): increased polymerization processivity and enhanced sensitivity to pyrophosphate of the mutant viral reverse transcriptase. Biochemistry 37:15908-15917.

4. Arion, D., N. Sluis-Cremer, and M. A. Parniak. 2000. Mechanism by which phosphonoformic acid resistance mutations restore 3'- azido-3'-deoxythymidine (AZT) sensitivity to AZT-resistant HIV-1 reverse transcriptase. J. Biol. Chem. 275:9251-9255.

5. Bakhanashvili, M., O. Avidan, and A. Hizi. 1996. Mutational studies of human immunodeficiency virus type 1 reverse transcriptase: the involvement of residues 183 and 184 in the fidelity of DNA synthesis. FEBS Lett. 391:257-262.

6. Balzarini, J. 1999. Suppression of resistance to drugs targeted to human immunodeficiency virus reverse transcriptase by combination therapy. Biochem Pharmacol 58:1-27.

7. Beard, W. A., S. J. Stahl, H. R. Kim, K. Bebenek, A. Kumar, M. P. Strub, S. P. Becerra, T. A. Kunkel, and S. H. Wilson. 1994. Structure/function studies of human immunodeficiency virus type 1 reverse transcriptase. Alanine scanning 
mutagenesis of an alpha-helix in the thumb subdomain. J. Biol. Chem.

269:28091-28097.

8. Bebenek, K., W. A. Beard, J. R. Casas-Finet, H. R. Kim, T. A. Darden, S. H.

Wilson, and T. A. Kunkel. 1995. Reduced frameshift fidelity and processivity of HIV-1 reverse transcriptase mutants containing alanine substitutions in helix $\mathrm{H}$ of the thumb subdomain. J. Biol. Chem. 270:19516-19523.

9. Boucher, C. A., N. Cammack, P. Schipper, R. Schuurman, P. Rouse, M. A. Wainberg, and J. M. Cameron. 1993. High-level resistance to (-) enantiomeric 2'-deoxy-3'-thiacytidine in vitro is due to one amino acid substitution in the catalytic site of human immunodeficiency virus type 1 reverse transcriptase. Antimicrob. Agents Chemother. 37:2231-2234.

10. Boucher, C. A., E. O'Sullivan, J. W. Mulder, C. Ramautarsing, P. Kellam, G. Darby, J. M. Lange, J. Goudsmit, and B. A. Larder. 1992. Ordered appearance of zidovudine resistance mutations during treatment of 18 human immunodeficiency virus-positive subjects. J. Infect. Dis. 165:105-110.

11. Bowman, R. R., W. S. Hu, and V. K. Pathak. 1998. Relative rates of retroviral reverse transcriptase template switching during RNA- and DNA-dependent DNA synthesis. J. Virol. 72:5198-5206.

12. Boyer, P. L., and S. H. Hughes. 1995. Analysis of mutations at position 184 in reverse transcriptase of human immunodeficiency virus type 1. Antimicrob. Agents Chemother. 39:1624-1628. 
13. Brosius, S., F. Grosse, and G. Krauss. 1983. Subspecies of DNA polymerase alpha from calf thymus with different fidelity in copying synthetic templateprimers. Nucleic Acids Res. 11:193-202.

14. Coffin, J. M. 1995. HIV population dynamics in vivo: implications for genetic variation, pathogenesis, and therapy. Science 267:483-489.

15. Coffin, J. M., S. H. Hughes, and H. E. Varmus. 1997. Retroviruses. Cold Spring Harbor Laboratory Press, Cold Spring Harbor, NY.

16. Delviks, K. A., and V. K. Pathak. 1999. Effect of distance between homologous sequences and 3' homology on the frequency of retroviral reverse transcriptase template switching. J. Virol. 73:7923-7932.

17. Ding, J., K. Das, Y. Hsiou, S. G. Sarafianos, A. D. Clark, Jr., A. JacoboMolina, C. Tantillo, S. H. Hughes, and E. Arnold. 1998. Structure and functional implications of the polymerase active site region in a complex of HIV1 RT with a double-stranded DNA template- primer and an antibody Fab fragment at 2.8 A resolution. J. Mol. Biol. 284:1095-1111.

18. Drosopoulos, W. C., and V. R. Prasad. 1996. Increased polymerase fidelity of E89G, a nucleoside analog-resistant variant of human immunodeficiency virus type 1 reverse transcriptase. J. Virol. 70:4834-4838.

19. Eckert, K. A., and T. A. Kunkel. 1993. Fidelity of DNA synthesis catalyzed by human DNA polymerase alpha and HIV-1 reverse transcriptase: effect of reaction pH. Nucleic Acids Res. 21:5212-5220. 
20. Gao, G., and S. P. Goff. 1998. Replication defect of moloney murine leukemia virus with a mutant reverse transcriptase that can incorporate ribonucleotides and deoxyribonucleotides. J. Virol. 72:5905-5911.

21. Gao, Q., Z. Gu, M. A. Parniak, J. Cameron, N. Cammack, C. Boucher, and M. A. Wainberg. 1993. The same mutation that encodes low-level human immunodeficiency virus type 1 resistance to 2',3'-dideoxyinosine and 2',3'dideoxycytidine confers high-level resistance to the (-) enantiomer of 2',3'dideoxy-3'- thiacytidine. Antimicrob. Agents Chemother. 37:1390-1392.

22. Garces, J., and R. Wittek. 1991. Reverse-transcriptase-associated RNaseH activity mediates template switching during reverse transcription in vitro. Proc. R. Soc. Lond. B. Biol. Sci. 243:235-239.

23. Georgiadis, M. M., S. M. Jessen, C. M. Ogata, A. Telesnitsky, S. P. Goff, and W. A. Hendrickson. 1995. Mechanistic implications from the structure of a catalytic fragment of Moloney murine leukemia virus reverse transcriptase. Structure 3:879-892.

24. Gilboa, E., S. W. Mitra, S. Goff, and D. Baltimore. 1979. A detailed model of reverse transcription and tests of crucial aspects. Cell 18:93-100.

25. Glickman, B. W., V. A. Saddi, and J. Curry. 1994. International Commission for Protection Against Environmental Mutagens and Carcinogens. Spontaneous mutations in mammalian cells. Mutat. Res. 304:19-32.

26. Gutierrez-Rivas, M., A. Ibanez, M. A. Martinez, E. Domingo, and L. Menendez-Arias. 1999. Mutational analysis of Phe160 within the "palm" 
subdomain of human immunodeficiency virus type 1 reverse transcriptase. J. Mol. Biol. 290:615-625.

27. Halvas, E. K., E. S. Svarovskaia, and V. K. Pathak. 2000. Development of an in vivo assay to identify structural determinants in murine leukemia virus reverse transcriptase important for fidelity. J. Virol. 74:312-319.

28. Halvas, E. K., E. S. Svarovskaia, and V. K. Pathak. 2000. The role of murine leukemia virus reverse transcriptase dNTP-binding site in retroviral replication and in vivo fidelity (submitted to J.Virol.).

29. Hu, W. S., E. H. Bowman, K. A. Delviks, and V. K. Pathak. 1997. Homologous recombination occurs in a distinct retroviral subpopulation and exhibits high negative interference. J. Virol. 71:6028-6036.

30. Hu, W. S., and H. M. Temin. 1990. Retroviral recombination and reverse transcription. Science 250:1227-1233.

31. Huang, H., R. Chopra, G. L. Verdine, and S. C. Harrison. 1998. Structure of a covalently trapped catalytic complex of HIV-1 reverse transcriptase: implications for drug resistance [see comments]. Science 282:1669-1675.

32. Jacobo-Molina, A., J. Ding, R. G. Nanni, A. D. Clark, Jr., X. Lu, C. Tantillo, R. L. Williams, G. Kamer, A. L. Ferris, P. Clark, and et al. 1993. Crystal structure of human immunodeficiency virus type 1 reverse transcriptase complexed with double-stranded DNA at 3.0 A resolution shows bent DNA. Proc. Natl. Acad. Sci. USA 90:6320-6324. 
33. Jonckheere, H., E. De Clercq, and J. Anne. 2000. Fidelity analysis of HIV-1 reverse transcriptase mutants with an altered amino-acid sequence at residues Leu74, Glu89, Tyr115, Tyr183 and Met184. Eur J Biochem 267:2658-2665.

34. Julias, J. G., D. Hash, and V. K. Pathak. 1995. E- vectors: development of novel self-inactivating and self-activating retroviral vectors for safer gene therapy. J. Virol. 69:6839-6846.

35. Keulen, W., N. K. Back, A. van Wijk, C. A. Boucher, and B. Berkhout. 1997. Initial appearance of the 184Ile variant in lamivudine-treated patients is caused by the mutational bias of human immunodeficiency virus type 1 reverse transcriptase. J. Virol. 71:3346-3350.

36. Kim, B., T. R. Hathaway, and L. A. Loeb. 1998. Fidelity of mutant HIV-1 reverse transcriptases: interaction with the single-stranded template influences the accuracy of DNA synthesis. Biochemistry 37:5831-5839.

37. Kim, T., R. A. Mudry, Jr., C. A. Rexrode, 2nd, and V. K. Pathak. 1996. Retroviral mutation rates and A-to-G hypermutations during different stages of retroviral replication. J. Virol. 70:7594-7602.

38. Kohlstaedt, L. A., J. Wang, J. M. Friedman, P. A. Rice, and T. A. Steitz. 1992. Crystal structure at 3.5 A resolution of HIV-1 reverse transcriptase complexed with an inhibitor. Science 256:1783-1790.

39. Kunkel, T. A., R. K. Hamatake, J. Motto-Fox, M. P. Fitzgerald, and A. Sugino. 1989. Fidelity of DNA polymerase I and the DNA polymerase I-DNA primase complex from Saccharomyces cerevisiae. Mol. Cell. Biol. 9:4447-4458. 
40. Luo, G. X., and J. Taylor. 1990. Template switching by reverse transcriptase during DNA synthesis. J. Virol. 64:4321-4328.

41. Mansky, L. M., and H. M. Temin. 1995. Lower in vivo mutation rate of human immunodeficiency virus type 1 than that predicted from the fidelity of purified reverse transcriptase. J. Virol. 69:5087-5094.

42. Mansky, L. M., and H. M. Temin. 1994. Lower mutation rate of bovine leukemia virus relative to that of spleen necrosis virus. J. Virol. 68:494-499.

43. Martinez, M. A., J. P. Vartanian, and S. Wain-Hobson. 1994.

Hypermutagenesis of RNA using human immunodeficiency virus type 1 reverse transcriptase and biased dNTP concentrations. Proc. Natl. Acad. Sci. USA 91:11787-11791.

44. Martin-Hernandez, A. M., E. Domingo, and L. Menendez-Arias. 1996. Human immunodeficiency virus type 1 reverse transcriptase: role of Tyr115 in deoxynucleotide binding and misinsertion fidelity of DNA synthesis. Embo J. 15:4434-4442.

45. Martin-Hernandez, A. M., M. Gutierrez-Rivas, E. Domingo, and L. Menendez-Arias. 1997. Mispair extension fidelity of human immunodeficiency virus type 1 reverse transcriptases with amino acid substitutions affecting Tyr115. Nucleic Acids Res. 25:1383-1389.

46. Mercoyrol, L., Y. Corda, C. Job, and D. Job. 1992. Accuracy of wheat-germ RNA polymerase II. Eur. J. Biocem. 206:49-58.

47. Najmudin, S., M. L. Cote, D. Sun, S. Yohannan, S. P. Montano, J. Gu, and M. M. Georgiadis. 2000. Crystal structures of an N-terminal fragment from 
Moloney murine leukemia virus reverse transcriptase complexed with nucleic acid: functional implications for template-primer binding to the fingers domain. J. Mol. Biol. 296:613-632.

48. Nijhuis, M., R. Schuurman, D. de Jong, R. van Leeuwen, J. Lange, S. Danner, W. Keulen, T. de Groot, and C. A. Boucher. 1997. Lamivudineresistant human immunodeficiency virus type 1 variants $(184 \mathrm{~V})$ require multiple amino acid changes to become co-resistant to zidovudine in vivo. J. Infect. Dis. 176:398-405.

49. Omer, C. A., K. Pogue-Geile, R. Guntaka, K. A. Staskus, and A. J. Faras. 1983. Involvement of directly repeated sequences in the generation of deletions of the avian sarcoma virus src gene. J. Virol. 47:380-382.

50. Oude Essink, B. B., N. K. Back, and B. Berkhout. 1997. Increased polymerase fidelity of the 3TC-resistant variants of HIV-1 reverse transcriptase. Nucleic Acids Res. 25:3212-3217.

51. Parthasarathi, S., A. Varela-Echavarria, Y. Ron, B. D. Preston, and J. P. Dougherty. 1995. Genetic rearrangements occurring during a single cycle of murine leukemia virus vector replication: characterization and implications. J. Virol. 69:7991-8000.

52. Pathak, V. K., and W. S. Hu. 1997. "Might as well jump!" Template switching by retroviral reverse transcriptase, defective genome formation, and recombination. Sem. Virol. 8:141-150.

53. Pathak, V. K., and H. M. Temin. 1990. Broad spectrum of in vivo forward mutations, hypermutations, and mutational hotspots in a retroviral shuttle vector 
after a single replication cycle: deletions and deletions with insertions. Proc. Natl. Acad. Sci. USA 87:6024-6028.

54. Pathak, V. K., and H. M. Temin. 1990. Broad spectrum of in vivo forward mutations, hypermutations, and mutational hotspots in a retroviral shuttle vector after a single replication cycle: substitutions, frameshifts, and hypermutations. Proc. Natl. Acad. Sci. USA 87:6019-6023.

55. Preston, B. D., B. J. Poiesz, and L. A. Loeb. 1988. Fidelity of HIV-1 reverse transcriptase. Science 242:1168-1171.

56. Reyland, M. E., and L. A. Loeb. 1987. On the fidelity of DNA replication. Isolation of high fidelity DNA polymerase-primase complexes by immunoaffinity chromatography. J. Biol. Chem. 262:10824-10830.

57. Roberts, J. D., K. Bebenek, and T. A. Kunkel. 1988. The accuracy of reverse transcriptase from HIV-1. Science 242:1171-1173.

58. Roberts, J. D., B. D. Preston, L. A. Johnston, A. Soni, L. A. Loeb, and T. A. Kunkel. 1989. Fidelity of two retroviral reverse transcriptases during DNAdependent DNA synthesis in vitro. Mol. Cell. Biol. 9:469-476.

59. Rubinek, T., M. Bakhanashvili, R. Taube, O. Avidan, and A. Hizi. 1997. The fidelity of 3' misinsertion and mispair extension during DNA synthesis exhibited by two drug-resistant mutants of the reverse transcriptase of human immunodeficiency virus type 1 with Leu74-->Val and Glu89-->Gly. Eur J Biochem 247:238-247.

60. Sarafianos, S. G., K. Das, A. D. Clark, Jr., J. Ding, P. L. Boyer, S. H. Hughes, and E. Arnold. 1999. Lamivudine (3TC) resistance in HIV-1 reverse 
transcriptase involves steric hindrance with beta-branched amino acids. Proc. Natl. Acad. Sci. USA 96:10027-10032.

61. Sarafianos, S. G., V. N. Pandey, N. Kaushik, and M. J. Modak. 1995. Glutamine 151 participates in the substrate dNTP binding function of HIV-1 reverse transcriptase. Biochemistry 34:7207-7216.

62. Schinazi, R. F., R. M. Lloyd, Jr., M. H. Nguyen, D. L. Cannon, A. McMillan, N. Ilksoy, C. K. Chu, D. C. Liotta, H. Z. Bazmi, and J. W. Mellors. 1993. Characterization of human immunodeficiency viruses resistant to oxathiolanecytosine nucleosides. Antimicrob. Agents Chemother. 37:875-881.

63. Shah, F. S., K. A. Curr, M. E. Hamburgh, M. A. Parniak, H. Mitsuya, J. G. Arnez, and V. R. Prasad. 2000. Differential influence of nucleoside analogresistance mutations $\mathrm{K} 65 \mathrm{R}$ and $\mathrm{L} 74 \mathrm{~V}$ on the overall mutation rate and error specificity of human immunodeficiency virus type 1 reverse transcriptase. J. Biol. Chem.

64. Shinnick, T. M., R. A. Lerner, and J. G. Sutcliffe. 1981. Nucleotide sequence of Moloney murine leukaemia virus. Nature 293:543-548.

65. Smerdon, S. J., J. Jager, J. Wang, L. A. Kohlstaedt, A. J. Chirino, J. M. Friedman, P. A. Rice, and T. A. Steitz. 1994. Structure of the binding site for nonnucleoside inhibitors of the reverse transcriptase of human immunodeficiency virus type 1. Proc. Natl. Acad. Sci. USA 91:3911-3915.

66. Smith, R. A., K. M. Remington, R. M. Lloyd, Jr., R. F. Schinazi, and T. W. North. 1997. A novel Met-to-Thr mutation in the YMDD motif of reverse 
transcriptase from feline immunodeficiency virus confers resistance to oxathiolane nucleosides. J. Virol. 71:2357-2362.

67. Taube, R., O. Avidan, and A. Hizi. 1997. The fidelity of misinsertion and mispair extension throughout DNA synthesis exhibited by mutants of the reverse transcriptase of human immunodeficiency virus type 2 resistant to nucleoside analogs. Eur J Biochem 250:106-114.

68. Telesnitsky, A., and S. P. Goff. 1993. RNase H domain mutations affect the interaction between Moloney murine leukemia virus reverse transcriptase and its primer-template. Proc. Natl. Acad. Sci. USA 90:1276-1280.

69. Temin, H. M. 1976. The DNA provirus hypothesis. Science 192:1075-1080.

70. Temin, H. M. 1993. Retrovirus variation and reverse transcription: abnormal strand transfers result in retrovirus genetic variation. Proc. Natl. Acad. Sci. USA 90:6900-6903.

71. Tisdale, M., S. D. Kemp, N. R. Parry, and B. A. Larder. 1993. Rapid in vitro selection of human immunodeficiency virus type 1 resistant to 3'-thiacytidine inhibitors due to a mutation in the YMDD region of reverse transcriptase. Proc. Natl. Acad. Sci. USA 90:5653-5656.

72. Wainberg, M. A. 1997. Increased fidelity of drug-selected M184V mutated HIV1 reverse transcriptase as the basis for the effectiveness of 3TC in HIV clinical trials. Leukemia 11 Suppl 3:85-88.

73. Wain-Hobson, S., P. Sonigo, O. Danos, S. Cole, and M. Alizon. 1985. Nucleotide sequence of the AIDS virus, LAV. Cell 40:9-17. 
74. Weiss, R. A., and H. W. Jaffe. 1990. Duesberg, HIV and AIDS [published erratum appears in Nature 1990 Jul 5;346(6279):22]. Nature 345:659-660. 


\section{CHAPTER 2}

\section{STRUCTURAL DETERMINANTS OF MURINE LEUKEMIA VIRUS REVERSE TRANSCRIPTASE THAT AFFECT THE FREQUENCY OF TEMPLATE SWITCHING}

EVGUENIA S. SVAROVSKAIA,${ }^{1,3}$ KRISTA A. DELVIKS,${ }^{3 \dagger}$ CAREY K. HWANG $, 2,3 \dagger$ and VINAY K. PATHAK ${ }^{3}$

Department of Biochemistry, ${ }^{I}$ Department of Microbiology and Immunology, ${ }^{2}$ West Virginia University, Morgantown, West Virginia 26506, and HIV Drug Resistance Program, National Cancer Institute, FCRDC, Frederick, Maryland $21702^{3}$

Experiments, described in this chapter, are published as a manuscript in Journal of Virology, Aug. 2000, 74(15):7171-8. 


\begin{abstract}
Retroviral reverse transcriptases (RTs) frequently switch templates within the same RNA (intramolecular) or between copackaged viral RNAs (intermolecular). Template switching by RT is necessary for completion of reverse transcription and results in the generation of mutations and recombination, which increase variation in retroviral populations. The structural features of retroviral RTs that affect the frequency of template switching are currently unknown. To identify structural elements of murine leukemia virus (MLV) RT important for template switching, we developed an in vivo assay. This assay used a retroviral vector encoding directly repeated sequences composed of overlapping fragments of the green fluorescent protein gene (GFP) that was permitted to undergo one round of reverse transcription. Template switching within the direct repeats functionally reconstituted GFP. We quantified the effect of mutations in RT on the frequencies of template switching. Using MLV RTs containing single amino acid substitution mutations in the Tyr-X-Asp-Asp (YXDD) motif, the dNTP binding site, the thumb domain, and the RNase H domain were characterized. The results showed that some mutations in the YXDD motif and dNTP binding site increased the frequency of template switching, while all mutations in the RNase $\mathrm{H}$ domain tested decreased the same frequency. Hydroxyurea treatment, which depleted nucleotide pools and reduced the rate of DNA synthesis, increased the frequency of template switching for the wild-type RT and the YXDD mutant RTs, but not for the RNase H mutant RTs. Based on these results, we proposed a dynamic copy-
\end{abstract}




\section{choice model in which both the rate of DNA polymerization and the rate of degradation of the RNA template influence the frequency of RT template switching.}

\section{INTRODUCTION}

The process of retroviral reverse transcription requires the dissociation of nascent DNA from one location on the RNA template and reassociation of the DNA at another region of homology (11). The first of these template-switching events, minus-strand DNA transfer, involves the transfer of the minus-strand strong-stop DNA from the $5^{\prime}$ end of the viral genomic RNA to the $3^{\prime}$ end of the RNA using the two identical R regions at the ends of the viral RNAs. The second template-switching event, plus-strand transfer, involves the transfer of the plus-strand strong-stop DNA using the complementarity between the primer tRNA and the primer binding site. It has been hypothesized that because these template-switching events are necessary for the completion of viral replication, retroviral RTs have evolved to possess low template affinity and low processivity (63). Since RTs possess low processivity, they also frequently undergo other internal template-switching events during reverse transcription. Intermolecular templateswitching events between copackaged viral RNAs can result in homologous and nonhomologous recombination $(24,35,64)$. On the other hand, intramolecular templateswitching events (within the same template) generate mutations such as deletions, deletions with insertions, and duplications $(44,46)$.

Retroviral vectors containing directly repeated sequences provide a powerful in vivo experimental model system for elucidating the mechanism of RT template switching $(14,32,46,70)$. Directly repeated sequences delete at a high frequency $(8,12,13,26$, 
$35,42,46,47,67)$, which appears to be correlated with the size of the repeats $(13,32,48$,

71). Deletion of direct repeats is a highly accurate process, because drug resistance genes and other selectable markers are functionally reconstituted with a high efficiency (13, 32). It was also recently shown that the linear distance between direct repeats increased the frequency of deletions and that within a 701-bp direct repeat, the frequency of template switching was higher near the $5^{\prime}$ end of the repeat than the $3^{\prime}$ end (14). These results suggested that the length of homology $3^{\prime}$ to the site of polymerization was important for efficient template switching. These data also suggested that degradation of the template RNA with RNase H permitted base pairing between the repeated sequence to facilitate RT template switching and deletion.

Structural features of RTs that may play an important role in their templateswitching properties are currently unknown. Previous studies have indicated that RNase $\mathrm{H}$ activity is necessary for minus-strand transfer as well as plus-strand transfer $(16,54$, 60, 61). Mutations in several regions of RT have been shown to affect processivity of DNA synthesis in vitro; these regions include the Tyr-X-Asp-Asp (YXDD) motif $(9,22$, $53)$, the thumb region $(2,3,5)$, the fingers domain $(52,59)$, residue $\mathrm{Q} 151$ of the dNTP binding site (30), and the RNase H domain $(1,62)$. In addition, other viral proteins, specifically, the nucleocapsid protein (NC) might be important for template switching. Some in vitro studies have suggested that NC increases the processivity of RT $(15,29)$. However, other studies have indicated that $\mathrm{NC}$ has no effect on the processivity of RT $(49,50)$.

To identify the structural determinants of RT that are important for template switching, we introduced amino acid substitutions in the murine leukemia virus (MLV) 
RT dNTP binding site, the YXDD motif, the $\alpha$-helix $H$ of the thumb domain, and the RNase H domain. We also developed an in vivo assay and determined the extent to which the mutations in RT affected the frequency of template switching. These results show that the dNTP binding site, the YXDD motif, the $\alpha$-helix $H$ of the thumb domain, and the RNase $\mathrm{H}$ domain of RT can influence template switching.

\section{MATERIALS AND METHODS}

Plasmids. Plasmid pLGPS expressed the MLV gag and pol genes from a truncated MLV long terminal repeat ( $\triangle$ LTR) promoter (41). The plasmid pSV-A-MLV$e n v$, which expressed the amphotropic MLV envelope gene from the long terminal repeat (LTR) promoter and SV40 enhancer, was obtained from the AIDS Research and Reference Reagents Program (39). The plasmid pBSpac encoded the puromycin Nacetyltransferase gene and conferred resistance to puromycin (66). The plasmid pSV $\alpha 3.6$ encoded the $\alpha$ subunit of the murine $\mathrm{Na}^{+}, \mathrm{K}^{+}$-ATPase gene and conferred resistance to ouabain (36). Plasmid pGA-1, a MLV-based retroviral vector, encoded the neomycin phosphotransferase gene (neo) as well as the $\beta$-galactosidase gene (33).

Construction of retroviral vector pES-GFFP. The MLV-based retroviral vector pES-GFFP was constructed using standard cloning procedures (51). Plasmid $\mathrm{pGF}_{100} \mathrm{FP}-\mathrm{Mlu}$ was constructed from $\mathrm{pGF}_{100} \mathrm{FP}$. The construction of $\mathrm{pGF}_{100} \mathrm{FP}$ was based on the parent vector pWH390-Cla (13). Plasmid pWH390-Cla was digested with EcoRI and a polylinker containing BglII, NruI, BamHI, StuI, and SalI was inserted, which regenerated the EcoRI site at the 3' end, forming pCH-L1. Plasmid pGL-1 [pGreen Lantern-1, encoding the green fluorescent protein (GFP)] (Gibco BRL) was digested with SpeI and BstBI, and the 363-bp fragment designated GF was inserted into the NruI site in 
pCH-L1, generating pVVCH-GF. The FP fragment (486 bp) containing a 100-bp direct repeat with GF was PCR amplified from pGL-1 (primer sequences available upon request). The FP fragment was inserted into the StuI site in pVVCH-GF generating pGF $_{100} \mathrm{FP}$. To construct pGF $\mathrm{p}_{100} \mathrm{FP}-\mathrm{Mlu}, \mathrm{pGF}_{100} \mathrm{FP}$ was partially digested with BamHI and a linker containing a unique $M l u \mathrm{I}$ site flanked by two $\mathrm{XhoI}$ sites was inserted between the GF and the FP fragments. Plasmid $\mathrm{pGF}_{100} \mathrm{FP}-\mathrm{Mlu}$ was cut with $B g l \mathrm{II}$ plus $M l u \mathrm{I}$ and treated with calf intestinal phosphatase plus the Klenow fragment of DNA polymerase I to generate dephosphorylated blunt ends. Plasmid pGL-1 was cut with HincII, and the resulting 521-bp fragment was ligated into the backbone vector. The correct structure of the resulting pES-GFFP plasmid was confirmed through restriction enzyme mapping.

Generation of MLV RT mutants. Construction of the dNTP binding site, YXDD motif, and the RNase H mutants of MLV RT was described previously (20, 21). A detailed description of the mutagenic oligonucleotides and the strategies used to introduce mutations in the $\alpha$-helix $\mathrm{H}$ of the thumb domain is available in Appendix 2. Briefly, the mutagenic oligonucleotides were designed to introduce additional silent mutations and generate new restriction sites. Restriction digestion analysis was performed to identify plasmids containing mutations, and DNA fragments containing the mutations were subcloned into pLGPS. The inserted fragments were analyzed by DNA sequencing to verify the presence of the desired mutation and the absence of undesired mutations (ALF Automated Sequencer, Pharmacia).

Cells, transfections, and infections. D17 dog osteosarcoma cells (obtained from American Type Culture Collection) were transfected, infected, and selected for resistance to ouabain or G418 (a neomycin analog) as previously described $(32,33)$. All 
cotransfections were performed using a 10:1 molar ratio of the plasmid of interest to the plasmid encoding the selectable marker. The D17 cells were selected for resistance to puromycin (final concentration, $3.2 \times 10^{-6} \mathrm{M}$ ). The D17-derived B2-1GFFP cells were maintained, transfected, and selected for drug resistance in a similar manner. PG13 cells (obtained from American Type Culture Collection) express MLV gag-pol and gibbon ape leukemia virus env (41). D17, D17-derived, and PG13 cells were maintained in Dulbecco's modified Eagle's medium (DMEM) supplemented with 6\% (D17) or 10\% (PG13) bovine calf serum, penicillin (50 U/ml), and streptomycin $(50 \mathrm{mg} / \mathrm{ml})$.

Hydroxyurea (HU) treatment was performed as previously described (34). Briefly, D17 cells were placed on culture media containing $1 \mathrm{mM} \mathrm{HU} 4 \mathrm{~h}$ prior to infection, $4 \mathrm{~h}$ during infection, and $24 \mathrm{~h}$ post-infection.

Protocol for determining the efficiency of RT template switching in vivo. B21 GFFP cells were plated at a density of $2 \times 10^{5}$ cells per 60 -mm diameter dish, and $24 \mathrm{~h}$ later were cotransfected with wild-type or mutated pLGPS and pSV $\alpha 3.6$. The transfected cells were selected for resistance to ouabain and the resistant colonies were pooled and expanded. After transfection with the pLGPS constructs, the B2-1GFFP cells were maintained in the presence of $1 \mu \mathrm{M} 3^{\prime}$-azido-3'-deoxythymidine (AZT) to reduce the probability of reinfection of the virus-producing cells. This concentration of AZT was previously shown to inhibit MLV replication 100-fold (33). Before collecting virus, the culture media containing AZT was removed and the cells were plated a density of $5 \times 10^{6}$ cells per 100-mm diameter dish. The culture media was replaced with fresh media $24 \mathrm{~h}$ later. Another $24 \mathrm{~h}$ later, the culture media containing ES-GFFP virus was harvested and used to infect D17 target cells. 
The infected D17 cells that were resistant to G418 were pooled and analyzed by fluorescence-activated cell scanning (FACS).

Southern blot analysis of proviral DNA. Genomic DNA was isolated from the B2-1GFFP cell line, and the proviral DNA structure was analyzed by Southern blot hybridization using standard procedures $(13,51)$. A 1.3-kb HindIII fragment of pVP212 (47) containing neo was used to generate a probe (specific activity, $4.8 \times 10^{9} \mathrm{cpm} / \mu \mathrm{g}$ ) as previously described (13).

\section{RESULTS}

Construction of B2-1GFFP cells. The MLV-based retroviral vector pES-GFFP and the MLV env expression construct pSV-A-MLV-env (Fig. 1A) were introduced into D17 cells to generate the B2-1GFFP cells (Fig. 1B). The vector pES-GFFP contained all cis-acting elements needed for viral replication, which included the LTRs, the MLV encapsidation signal $(\Psi)$, the primer-binding site, and the polypurine tract. In addition, the vector encoded overlapping GF and FP fragments of GFP separated by $25 \mathrm{bp}$. The directly repeated sequence (the "F" portion) was $250 \mathrm{bp}$ in length. During reverse transcription, the directly repeated sequence was deleted at a high frequency, resulting in the reconstitution of a functional GFP gene (Fig. 1A). Plasmid pES-GFFP also contained the selectable marker neo. The GFFP and neo were expressed from a single RNA transcript initiating in the $5^{\prime}$ LTR. An internal ribosomal entry site (IRES) of encephalomyocarditis virus was used to translate neo $(27,28,31)$.

To construct the B2-1GFFP cell line, the pSV-A-MLV-env was cotransfected with pBSpac into D17 cells. The transfected cells were selected for resistance to puromycin and 10 cell clones were isolated and expanded. To verify that the cell clones 
expressed a functional amphotropic MLV envelope, the cell clones were cotransfected with the construct pLGPS that expressed the MLV gag-pol as well as the retroviral vector pGA-1 that encoded neo (Fig. 1A) (20). G418 resistant cells were selected and virus produced from these cells was used to infect fresh D17 cells. The infected D17 cells were selected for resistance to G418 to determine virus titers. The B2 cell clone expressing amphotropic MLV envelope was selected because it exhibited the highest viral titer $\left(10^{5} \mathrm{CFU} / \mathrm{ml}\right)$.

To introduce the pES-GFFP vector into B2 cells, pES-GFFP was first transfected into the MLV-based packaging cell line PG13, and G418-resistant cells were selected. Virus harvested from the pool of transfected PG13 cells was used to infect B2 cells. After selection for G418 resistance, five individual B2 cell clones were isolated and expanded. To select a clone with undeleted directly repeated F portions of GFP, all five clones were analyzed by FACS. Analyses of four of the five clones indicated that less than $0.4 \%$ of the cells were fluorescent. The proportion of the cells that were fluorescent was similar to the proportion of fluorescent cells in uninfected D17 cells $(0.1 \%)$. FACS analysis of one of the clones, named B2-1, is shown in Fig. 1C (upper panel). The similarity in fluorescence compared to uninfected cells indicated that the ES-GFFP provirus did not undergo direct repeat deletion during infection of the B2 cells. To verify that the ES-GFFP provirus was capable of completing one cycle of retroviral replication, cell clones with undeleted direct repeats were cotransfected with pLGPS and pSVa3.6. Ouabain-resistant cells were selected and virus produced was used to infect fresh D17 cells, which were selected for resistance to G418 and analyzed by FACS. The B2-1 cell clone that exhibited the highest titer $\left(10^{5} \mathrm{CFU} / \mathrm{ml}\right)$ was named B2-1GFFP and used in all 
subsequent experiments (data not shown). Representative FACS analysis of D17 target cells infected with virus collected from the B2-1GFFP cells transfected with pLGPS and selected for G418 resistance is shown in Fig. 1C (lower panel). A total of 11 experiments that were subsequently performed with the wild-type RT (Table 1) indicated that the deletion frequency was highly reproducible $(10.4 \% \pm 0.4 \%)$. The presence of a high proportion of fluorescent cells indicated that the B2-1GFFP cells contained a functional ES-GFFP provirus, which was capable of undergoing direct repeat deletion during reverse transcription. Control experiments performed with virus producing cells that were maintained in the absence and presence of AZT to reduce the probability of reinfection of the virus producer cells. The experiments indicated that maintaining the virus producing cells in the presence of AZT did not influence the frequency of direct repeat deletion and GFP reconstitution (data not shown).

It was also important to show that the B2-1GFFP cells did not express a replication-competent MLV, the presence of which could potentially result in multiple rounds of ES-GFFP replication. ES-GFFP virus collected from the B2-1GFFP cells transfected with pLGPS was used to infect D17 cells. After G418 selection, resistant colonies were pooled. Culture supernatant from a pool of infected D17 cells was used to infect fresh D17 cells, which were then selected for resistance to G418. The absence of G418 resistant colonies indicated that the B2-1GFFP cell line did not harbor a replication competent virus (data not shown).

To ensure that the B2-1GFFP cells contained only one ES-GFFP provirus, genomic DNA was extracted from the B2-1GFFP cells and analyzed by Southern blot hybridization (Fig. 2). The DNA was digested with either BamHI or EcoRI and 
hybridized to a neo-specific probe. Each of the enzymes (BamHI and EcoRI) cut only once in the ES-GFFP provirus, generating one fragment representing the $3^{\prime}$ portions of the provirus and flanking regions. The presence of only a single detectable fragment for each digestion indicated that only one provirus was present in the B2-1GFFP cell line.

Direct repeat deletion assay. The MLV RT expression construct pLGPS was subjected to site-directed mutagenesis, and the B2-1GFFP cells were used to identify the protein determinants that were important for template switching during reverse transcription (Fig. 1A and B). First, pLGPS-derived constructs containing mutations in the MLV RT were separately introduced into the B2-1GFFP cells by cotransfection with pSVa3.6. Ouabain-resistant colonies were pooled and expanded. Virus was harvested from the pools of transfected cells and serially diluted virus was used to infect D17 target cells. The infected D17 cells were selected for resistance to G418 and the resulting colonies were pooled and analyzed by FACS to determine the frequency of direct repeat deletion. In most experiments, approximately 100 to 1,000 colonies were pooled for each analysis. In general, the multiplicity of infection was $<0.0005$, and the probability of double infection was very low $(<1 / 2000$ colonies). The frequency of direct repeat deletion provided a measure of the template-switching events during one cycle of retroviral replication. Using this assay, the effect of mutations introduced in the MLV RT on template switching during reverse transcription was determined.

All mutants that were tested in the direct repeat deletion assay are summarized in Fig. 3A. Two criteria were used for selection of the RT mutants for analysis of their effect on template switching. First, mutations that were previously reported to be important for the processivity or fidelity of reverse transcription were selected for the 
study. Second, only mutants that were previously shown to generate detectable viral titers were selected because the in vivo direct repeat deletion assay required that the mutants complete one cycle of retroviral replication.

\section{Effect of dNTP binding site and YXDD motif mutants on template switching.}

The amino acid residues of MLV RT involved in binding to the dNTP substrate were previously identified on the basis of sequence alignments and comparison of crystal structures of MLV and HIV-1 RTs (17, 21, 23, 25). Residues L151, F155, and F156 of MLV RT are homologous to residues V111, Y115, and F116 of HIV-1 RT, respectively (Fig. 3B). The effects of L151F, F155Y, F156W, and Q190M mutations on template switching were determined. The F155Y, F156W, and Q190M mutants exhibited an increase in the frequency of direct repeat deletions, which ranged from $14.6 \%$ to $49.5 \%$ (Table 1, Fig. 4A). However, the L151F mutation did not affect the ability of the RT to switch templates. The most significant change was displayed by the F156W mutant, which increased direct repeat deletion frequency 4.8-fold $(P<0.00005$; all statistical analysis was performed using the two-sample $t$ test).

Position V223 of the highly conserved YXDD motif of MLV RT was selected for mutagenesis because previous studies indicated that this amino acid was important for the processivity and the fidelity of $\mathrm{RT}(9,20,22,53)$. The results obtained from analysis of the V223I and V223M mutants are summarized in Table 1 and Fig. 4A. The V223I mutant of MLV RT exhibited a 2-fold increase in the frequency of GFP reconstitution ( $P$ $<0.00005)$. However, the V223M mutant of MLV RT did not exhibit a statistically significant alteration in the frequency of GFP reconstitution $(P=0.246)$. Therefore, 
substitution of the V223 of MLV RT with the equivalent methionine residue in HIV-1 RT did not change the frequency of template switching by MLV RT (Table 1, Fig. 4A).

Effect of thumb domain mutants on template switching. The thumb domain of MLV RT was chosen as a target of mutational analysis because the HIV-1 RT crystal structure and in vitro assays strongly suggested that it was important for processivity (2, 3, 5). Since the thumb domain of the MLV RT has not yet been crystallized and there is no significant primary sequence homology in this region of MLV and HIV-1 RTs, the precise location of the MLV RT $\alpha$-helix $H$ of the thumb domain was unclear. The sequence of the MLV RT and spleen necrosis virus (SNV) RT, which is similar in primary sequence to the MLV RT, was compared to localize the MLV RT $\alpha$-helix H of the thumb domain. Amino acid sequences that have maximum homology between MLV and SNV RTs, and are flanked by proline residues, were proposed to form an analog of the $\alpha$-helix H of the HIV-1 RT (Fig. 3C). Additionally, molecular modeling of the MLV RT thumb domain strongly suggests that this region is structurally equivalent to the $\alpha$ helix H of the HIV-1 RT (58).

The thumb domain of MLV RT contains residues G305 and F309, which are hypothesized to be equivalent to the HIV-1 residues G262 and W266. This hypothesis predicts that residues R301, G305, and F309 face the same side of the $\alpha$-helix $\mathrm{H}$ and are involved in making contacts with the template-primer complex. Previously, several mutations were introduced at all three of these residues and their effect on the fidelity of reverse transcription was determined (58). For this study, the R301L, R301Q, R301S, F309A, F309H, and F309W mutants were selected for further analysis. None of the 
mutations at G305 could be tested, because mutations at this position resulted in undetectable viral titers (58).

The effects of mutations at residues R301 and F309 on the frequency of direct repeat deletion are summarized in Table 1 and Fig. 4B. The R301L and F309A mutants exhibited statistically significant reductions of $6.6 \%$ and $5.9 \%$, respectively, in the frequency of GFP reconstitution $(P<0.003)$. These mutants exhibited a deletion frequency that was $60 \%$ of the wild-type RT frequency (0.6-fold). Conversely, the F309H mutant exhibited a statistically significant increase in the frequency of direct repeat deletions to $180 \%$ of wild type (1.8-fold, $P<0.00005)$. Finally, the R301Q, R301S, and F309W mutants did not exhibit a statistically significant change in the frequency of direct repeat deletions. Therefore, mutations of residues R301 and F309 to the equivalent $\mathrm{Q}$ and W residues found in the HIV-1 RT, respectively, did not affect the frequency of RT template switching.

Effect of RNase H domain mutants on template switching. Mutations S526A, Y598V, and R657S were introduced into the MLV RNase H domain, and the effects of these mutations on template switching were determined. These mutations were selected because it was previously shown that the RNase $\mathrm{H}$ domain played an important role in obligatory template-switching events during reverse transcription $(16,54,60,61)$. Additionally, it was previously shown that these RNase $\mathrm{H}$ mutants permitted viral replication to occur. These mutants exhibited much slower replication kinetics, suggesting that there was a defect in RNase $\mathrm{H}$ activity $(6,7)$.

The effect of the S526A, Y598V, and R657S mutations on the frequency of direct repeat deletion are summarized in Table 1 and Fig. 4C. All three RNase H mutants 
exhibited a statistically significant reduction in the frequency of direct repeat deletions $(P$ $<0.00005)$. The frequency of deletions was reduced to approximately $50 \%$ of that observed for wild-type RT (0.5-fold).

\section{Effect of hydroxyurea (HU) treatment on the frequency of direct repeat}

deletion. HU treatment has been shown to deplete all four cellular nucleotide pools and increase retroviral mutation rates (34). It has been recently shown that HU treatment of infected cells resulted in a significant reduction in the rate of polymerization with which reverse transcriptions proceeds (48).

We hypothesize that mutations in the dNTP binding site and the YXDD motif result in polymerases that catalyze DNA synthesis more slowly than wild-type RT, and that this reduction in the rate of DNA synthesis increases the frequency of RT template switching. A model to explain the rationale behind this hypothesis is outlined in Fig. 6 and discussed later. To test this hypothesis, D17 target cells were infected with virus generated with the wild-type RT as well as V223I and Y598V mutants of RT in the absence or presence of $1 \mathrm{mM} \mathrm{HU}$. The frequency of direct repeat deletion and GFP reconstitution was determined. The results are shown in Fig. 5 and Table 2. In the presence of HU, the frequency of GFP reconstitution was increased by 1.7-fold for wildtype RT, which was in agreement with previously published results $(P<0.0065)(48)$. Similarly, the frequency of GFP reconstitution increased 1.8-fold for the V223I mutant in the presence of $\mathrm{HU}(P<0.0018)$, indicating that $\mathrm{HU}$ treatment could further increase the frequency of direct repeat deletion for mutants that exhibited a higher deletion frequency in the absence of $\mathrm{HU}$. However, $\mathrm{HU}$ treatment did not increase the frequency of direct repeat deletions for the Y598V RNase H mutant of MLV RT $(P=0.836)$. 


\section{DISCUSSION}

\section{A rapid and quantitative in vivo assay for $\mathbf{R T}$ template switching. The}

experiments described here demonstrate that we have developed a powerful in vivo assay to identify structural determinants of MLV RT that are important for template switching. This assay is more rapid than previously described assays utilizing the herpes simplex virus thymidine kinase or the $\beta$-galactosidase gene because it is not necessary to compare viral titers after different drug selections or count numerous colonies $(13,48)$.

Furthermore, the FACS analysis of infected cells allows accurate and rapid quantitation of the frequency of direct repeat deletion from large pools of infected cell colonies. However, one restriction of the assay is that it is not possible to determine the rate of template switching for mutants that are unable to complete one cycle of retroviral replication. Nevertheless, the assay makes it possible to rapidly analyze a large number of RT mutants and to determine whether the structural alterations affect the frequency of template switching during in vivo retroviral replication.

\section{Structural determinants of RT that are important for template switching.}

Our results demonstrate that several different domains of MLV RT can affect the frequency of template switching. Mutations in the dNTP binding site, the YXDD motif, the $\alpha$-helix $\mathrm{H}$ of the thumb domain, and the RNase $\mathrm{H}$ domain can affect the frequency of template switching. These results are not surprising, since previous studies have shown that mutations in these domains can affect RT processivity $(1-3,5,9,22,30,52,53,59$, 62). It was interesting to note that most of the mutations in the YXDD motif and the dNTP binding site increased the frequency of RT template switching. These mutations would be expected to interfere with the rate of DNA polymerization, although this effect 
has not been directly shown. This expectation is supported by the observation that most of these mutants displayed lower RT activities (21). The F156W mutant, which exhibited the largest increase in the frequency of RT template switching (4.8-fold), also exhibited the most severe defects in viral replication (2\% of the wild-type titer) and RT activity (11\% of the wild-type activity) (21). However, the frequency of RT template switching was not correlated to the reduction in viral titers for other mutants, perhaps because other steps in retroviral replication, such as initiation of DNA synthesis, were also affected by these mutations.

Previous studies have shown that alanine-scanning mutations in the $\alpha$-helix $\mathrm{H}$ of the thumb domain of HIV-1 RT greatly decreased in vitro fidelity and increased the rate of frameshift mutations $(4,5)$. These results suggested that mutations in the $\alpha$-helix $H$ would decrease processivity and increase the frequency of RT template switching. It was therefore surprising that most of the mutations tested in the present study either had no effect or decreased the frequency of RT template switching. The only exception was the F309H mutant, which increased the frequency of template switching by nearly 2-fold. It was especially surprising that the F309A mutant of MLV RT did not increase the frequency of template switching in vivo. In vitro studies and biochemical analysis of the equivalent mutant in HIV-1 RT (W266A) has shown that this mutant has a very low affinity for the template-primer and the dissociation constant is nearly 430-fold higher than that observed for the wild-type HIV-1 RT (4). The F309A mutation in MLV RT might affect other properties of the enzyme that prevent template switching despite its low template affinity. Mutations in the primer grip have been previously shown to 
reduce RNase $\mathrm{H}$ activity $(18,43)$. Therefore, it is possible that the F309A mutation in MLV RT also affects RNase H activity, which might suppress template switching.

All of the RNase H mutants tested exhibited lower frequencies of RT template switching. These results are consistent with previous observations that RNase $\mathrm{H}$ is essential for template switching in vitro as well as for obligatory strand transfer events during viral replication $(16,40,54,60,61)$. Interestingly, the RNase $\mathrm{H}$ mutants of MLV RT reduced the frequency of template switching by approximately $50 \%$. We have previously observed that RT template-switching events occur at very similar frequencies during RNA-dependent and DNA-dependent DNA synthesis (8). According to the previously proposed model (14), RNase $\mathrm{H}$ activity is necessary for template switching during RNA-dependent DNA synthesis. Therefore, the MLV RT RNase H mutants may severely impair template switching during RNA-dependent DNA synthesis but not affect the frequency of template switching during DNA-dependent DNA synthesis. If this interpretation is correct, then only a small fraction of the template switching events observed with the RNase H mutants of MLV RT occurred during minus-strand DNA synthesis.

Dynamic copy-choice model for RT template switching. We recently developed a model for RT template switching (14). The model proposed that basepairing between newly synthesized DNA sequences $3^{\prime}$ to the RT with complementary sequences of the template increases the probability of RT switching templates. As RT copies the $3^{\prime}$ direct repeat, RNase H degrades the RNA template $3^{\prime}$ to the RT. Next, hydrogen bonding occurs between the newly synthesized single-stranded DNA and complementary sequences in the $5^{\prime}$ copy of the direct repeat. The conformational 
rearrangements that permit this hydrogen bonding are depicted as a loop in the template RNA in Fig. 6. The hydrogen-bonding interactions $3^{\prime}$ to the RT serve to bring the homologous acceptor template in close proximity to the RT, subsequently leading to branch migration and a template switch.

We have modified the proposed model to incorporate observations reported in this study (Fig. 6). The revised model, called the dynamic copy-choice model, proposes that there is a steady state between the rate of DNA polymerization and the rate of template RNA degradation $3^{\prime}$ to the RT. The steady state determines the extent of nascent DNA that is available for base-pairing interactions with the acceptor template. We propose that this steady state can be disturbed by affecting the rate of DNA polymerization as well as the rate of RNA degradation. Once the nascent DNA $3^{\prime}$ to the RT is available for basepairing interactions with the acceptor template, the NC protein promotes hydrogen bonding and duplex formation (65). After the DNA duplex forms $3^{\prime}$ to the RT, the primer end of the nascent DNA is released from the donor template, which might involve its dissociation from the RT. Finally, the primer end associates with the acceptor template, completing the template switch. Even though the RT template-switching events are portrayed as intramolecular events, the same mechanistic events could also result in intermolecular template-switching events.

In this study, we observed that conditions likely to reduce the rate of DNA polymerization increased the frequency of RT template switching. These conditions include carrying out reverse transcription with HU treatment as well as RTs containing mutations in the dNTP binding site and the YXDD motif. The reduction in the rate of DNA polymerization may permit more efficient degradation of the template RNA and/or 
provide more time for hydrogen bond formation between the nascent DNA and the acceptor template. On the other hand, when RNase H mutants of RT were used, the rate of RNA degradation might have been reduced, resulting in impairment of base-pairing interactions between the nascent DNA and the acceptor template. As a result, the frequency of RT template switching was reduced.

The dynamic copy-choice model helps to integrate some of the previously proposed models and experimental observations associated with RT template switching. First, the previously proposed forced copy-choice model is consistent with the dynamic copy-choice model (10). The forced copy-choice model proposed that when RT encounters a break in the template RNA, the RT switches templates. In the dynamic copy-choice model, a break in the RNA would represent one extreme situation in the spectrum in which the rate of DNA polymerization has been reduced to zero. The obligatory template switches during reverse transcription, namely minus-strand and plusstrand DNA transfer, also represent the same situation in which the rate of DNA polymerization is zero. Second, the dynamic copy-choice model is consistent with several previous observations that secondary structures in the template RNA create RT pause sites, increasing the frequency of template switching $(37,38,45,55-57,69)$. Since RT pausing is likely to reduce the rate of DNA polymerization, its effect on RT template switching should be similar to that observed for HU treatment or RT mutants expected to reduce the rate of DNA polymerization.

Our observation that HU treatment did not increase the frequency of RT template switching for the RNase H mutant Y598V suggests that decreasing the rate of DNA polymerization is unable to overcome this particular defect in RNase $\mathrm{H}$ activity. 
Although at first glance this observation appears surprising with respect to the dynamic copy-choice model, the interpretation of the result is dependent on the extent to which the template-switching events observed with the Y598V mutant of MLV RT occurred during minus-strand DNA synthesis. The observation that HU treatment did increase the frequency of template switching for wild-type RT suggests that the rates of DNA polymerization and RNase $\mathrm{H}$ degradation are similar. It is possible that the $\mathrm{RNase} \mathrm{H}$ activity of the Y598V mutant of RT is substantially lower than the wild-type RNase H activity. If so, the HU treatment might not be able to compensate for this substantial RNase $\mathrm{H}$ defect by reducing the rate of DNA polymerization. As discussed earlier, only a small fraction of the template-switching events observed with this mutant may have occurred during minus-strand DNA synthesis. For example, if we assume that $25 \%$ of the template switching events observed with the Y598V mutant of MLV RT occurred during minus-strand DNA synthesis, then only $1 \%$ of the proviruses underwent direct repeat deletion during minus-strand DNA synthesis (25\% of an overall deletion frequency of 4\%). If the frequency of direct repeat deletion during minus-strand DNA synthesis is increased by approximately 2-fold with HU treatment, then the overall rate of direct repeat deletion would be expected to increase from $4 \%$ to $5 \%$. The direct repeat deletion assay used in this study may not be sufficiently sensitive to detect such a small increase in the deletion frequency.

It is also important to note that the size of the direct repeats might determine the sensitivity of the assay and whether a defect in RNase $\mathrm{H}$ activity can be overcome to a level that can be detected by reducing the rate of polymerization. It is possible that increasing the size of direct repeat will increase the time frame in which a defective 
RNase $\mathrm{H}$ can degrade the template RNA. Consequently, the frequency of RT template switching events that occur during minus-strand DNA synthesis will be increased for all RTs. Since the overall frequencies of direct repeat deletions will be higher, the overall sensitivity of the assay should be increased.

It must be pointed out that the Y598V mutant of RT was previously shown to have $100 \%$ polymerization-independent RNase $\mathrm{H}$ activity in an in vitro assay (7). However, it is unknown whether the polymerization dependent and/or the polymerization-independent activities of RNase $\mathrm{H}$ are important for RT template switching. Furthermore, it is unclear whether the in vitro RNase $\mathrm{H}$ activity reflects the level of activity that is displayed in the context of in vivo viral replication. The Y598V mutant of RT most likely has a defect in RNase $\mathrm{H}$ activity in vivo, since it exhibited slower kinetics of viral replication and a 5-fold reduction in viral titer $(6,20)$. Therefore, it is possible that the Y598V RNase $\mathrm{H}$ defect cannot be overcome by decreasing the rate of DNA polymerization with HU treatment.

Finally, other structural determinants of RT as well as other viral proteins not analyzed in this study might have a strong influence on RT template switching. Specifically, the NC protein has been shown to promote minus-strand and plus-strand DNA transfer events, and might play an important role in stabilizing the hydrogen bonding between the nascent DNA and acceptor template $(19,68)$. Experiments to analyze the role of other structural determinants of RT and $\mathrm{NC}$ in template switching are underway. 


\section{ACKNOWLEDGMENTS}

We especially thank Wei-Shau Hu for her valuable intellectual input and discussions throughout this project, Steve Hughes and John Coffin for valuable intellectual input and discussion of results, and Ann Arthur for her editorial expertise and revisions.

This work was supported by the Public Health Service grant CA58875 from the National Institutes of Health and by the HIV Drug Resistance Program, National Cancer Institute. 
TABLE 1. Effect of mutations in MLV RT on the frequency of direct repeat deletion and GFP reconstitution

\begin{tabular}{ccccc}
\hline $\begin{array}{c}\text { MLV RT } \\
\text { mutants }\end{array}$ & $\begin{array}{c}\text { No. of } \\
\text { experiments }\end{array}$ & $\begin{array}{c}\text { Total no. of } \\
\text { colonies }^{a}\end{array}$ & $\begin{array}{c}\text { Frequency of } \\
\text { direct repeat } \\
\text { deletions } \\
\text { (mean } \% \pm \text { SE) }\end{array}$ & $\begin{array}{c}\text { Relative change } \\
\text { in direct repeat } \\
\text { deletion } \\
\text { frequency }^{c}\end{array}$ \\
\hline Wild type & 11 & 7333 & $10.2 \pm 0.4$ & 1.0 \\
dNTP binding site & & & & \\
L151F & 3 & 530 & $9.2 \pm 0.1$ & No change \\
F155Y & 2 & 336 & $14.6 \pm 2.6$ & 1.4 \\
F156W & 6 & 544 & $49.5 \pm 5.4$ & 4.8 \\
Q190M & 3 & 444 & $18.4 \pm 2.1$ & 1.8 \\
& & & & \\
YXDD motif & & 1597 & $20.2 \pm 1.0$ & 2.0 \\
V223I & 6 & 297 & $9.2 \pm 0.1$ & No change \\
V223M & 3 & & & \\
& & & & 0.6 \\
Thumb domain & & 418 & $6.6 \pm 1.2$ & No change \\
R301L & 3 & 601 & $7.7 \pm 0.1$ & No change \\
R301Q & 2 & 517 & $9.2 \pm 1.6$ & 0.6 \\
R301S & 2 & 408 & $5.9 \pm 0.7$ & 1.8 \\
F309A & 6 & 265 & $18.0 \pm 1.0$ & No change \\
F309H & 2 & 832 & $11.9 \pm 2.0$ &
\end{tabular}

RNase H domain

$\begin{array}{lcccc}\text { S526A } & 6 & 2047 & 5.3 \pm 0.3 & 0.5 \\ \text { Y598V } & 12 & 5120 & 5.0 \pm 0.4 & 0.5 \\ \text { R657S } & 6 & 2099 & 5.3 \pm 0.5 & 0.5\end{array}$

${ }^{a}$ Total number of colonies analyzed by FACS in 2 to 12 independent infections.

${ }^{b}$ Frequency of direct repeat deletion was determined as a percent of infected D17 target cells that exhibited fluorescence after G418 selection compared to the negative control. The standard error of the mean (SE) was determined by using the Sigma Plot 5.0 program. 
${ }^{c}$ Calculated as follows: frequency of direct repeat deletion observed with mutant MLV RT $\div$ frequency of direct repeat deletion observed with the wild-type RT.

Statistically significant changes in the frequency of direct repeat deletions, relative to the wild-type RT (set to 1.0) are shown (two-sample $t$ test: $P<0.05$ ). 
TABLE 2. Effect of HU treatment on the frequency of direct repeat deletion and GFP reconstitution

\begin{tabular}{lcccc}
\hline MLV RT & $\begin{array}{c}\text { No. of } \\
\text { experiments }\end{array}$ & $\begin{array}{c}\text { Total no. of } \\
\text { colonies }^{a}\end{array}$ & $\begin{array}{c}\text { Frequency of } \\
\text { direct repeat } \\
\text { deletions } \\
\text { (mean } \% \pm \mathrm{SE})^{b}\end{array}$ & $\begin{array}{c}\text { Relative change } \\
\text { in direct repeat } \\
\text { deletion } \\
\text { frequency }^{c}\end{array}$ \\
\hline $\mathrm{WT}$ & 5 & 4392 & $9.9 \pm 0.7$ & 1.0 \\
$\mathrm{WT}+\mathrm{HU}$ & 6 & 632 & $17.2 \pm 1.8$ & 1.7 \\
& 3 & 755 & $18.0 \pm 1.3$ & 1.0 \\
V233I & 3 & 212 & $32.7 \pm 1.5$ & 1.8 \\
V233I + HU & 3 & 1059 & $4.0 \pm 0.3$ & 1.0 \\
Y598V & 3 & 450 & $3.9 \pm 0.8$ & 1.0 \\
Y598V + HU & 3 & & & \\
\hline
\end{tabular}

${ }^{a}$ Total number of colonies that were analyzed by FACS in 3 to 6 independent infections.

${ }^{b}$ Determined as for Table 1.

${ }^{c}$ Calculated as follows: frequency of direct repeat deletion observed with mutant or wildtype MLV RT $\div$ frequency of direct repeat deletion observed with the same MLV RT in the presence of $1 \mathrm{mM} \mathrm{HU}$. Statistical analysis using the two-sample $t$ test showed that the wild-type as well as the V233I mutant of MLV RT displayed direct repeat deletion frequencies different from that observed in the presence of $1 \mathrm{mM} \mathrm{HU}(P<0.01)$. The direct repeat deletion frequencies obtained with the Y598V mutant of MLV RT were not significantly different in the presence or absence of $\mathrm{HU}(P=0.836)$. 


\section{FIGURE LEGENDS AND FIGURES}

\section{FIG. 1. Structures of MLV-based constructs and direct repeat deletion}

assay to identify structural determinants of MLV RT important for template switching. (A) Structures of MLV-based vector pES-GFFP, pLGPS, and pSV-A-MLVenv. The pES-GFFP vector contains LTRs and all cis-acting elements of MLV. The GFFP and neo are transcribed from the LTR promoter. IRES of encephalomyocardidtis virus is used to express neo. The directly repeated F portion of GFP is shaded and indicated by overhead arrows. During reverse transcription, the repeated F portion may be deleted to reconstitute a functional GFP. The pLGPS construct expresses the MLV gag and pol from a truncated viral LTR. The pSV-A-MLV-env construct expresses the amphotropic MLV envelope from a truncated MLV LTR and the SV40 promoter enhancer. $\Psi$, MLV packaging signal. (B) Experimental protocol. B2-1GFFP, a D17based cell line expressing pES-GFFP and pSV-A-MLV-env, was constructed. The wildtype or mutated pLGPS constructs were separately cotransfected (Tf) with pSV $\alpha 3.6$ into the B2-1GFFP cells and the virus produced was used to infect (Inf) D17 cells. The infected cell clones resistant to G418 were analyzed by FACS and frequencies of direct repeat deletion were determined. (C) Top panel shows FACS analysis of B2-1GFFP cells. Bottom panel shows FACS analysis of D17 target cells infected with virus collected from the B2-1GFFP cells transfected with wild-type pLGPS and selected for G418 resistance. 
FIG. 2. Southern analysis of B2-1GFFP cells. Structure of ES-GFFP provirus is shown. Thick zig-zag lines represent host cell DNA flanking the provirus. The approximate locations of a $B a m \mathrm{HI}$ and an EcoRI restriction site in the provirus, and the expected lengths of the restriction fragments are shown. Genomic DNA isolated from B2-1GFFP cells was digested with either EcoRI (lane E) or BamHI (lane B). The blot was hybridized to a neo-specific probe (black bar).

FIG. 3. Mutants of MLV RT. (A) Selected regions of the MLV RT primary sequence containing the dNTP binding site, the YXDD motif, the $\alpha$-helix $H$ of the thumb domain, and the RNase $\mathrm{H}$ are shown and labeled. The numbers above the primary sequence indicate the amino acid positions in the primary sequence. The substitution mutations analyzed at each amino acid position are indicated below the primary sequence with downward arrows. (B) Primary sequence alignment of the MLV RT and HIV-1 RT dNTP binding sites. Double dots represent identical amino acids, whereas single dots represent conserved amino acids. The numbers indicate amino acid positions in the primary sequence. (C) Primary sequence alignment of the $\alpha$-helix H of the HIV-1 RT thumb domain and corresponding sequences in MLV and SNV RTs.

FIG. 4. Effect of mutations in the MLV RT dNTP binding site and YXDD motif (A), the $\alpha$-helix $H$ of the thumb domain (B), and the RNase $H$ domain (C) on the frequency of GFP reconstitution. The frequencies of GFP reconstitution were determined by FACS analysis and are shown for wild-type RT (open bars) and mutant RTs (shaded bars). The results represent and average of 2 to 12 experiments, and the error bars represent the standard error of the mean. 
FIG. 5. Effect of HU treatment on the frequencies of GFP reconstitution exhibited by the wild-type, V223I mutant, and Y598V mutant of MLV RT. The open and shaded bars represent the frequencies of GFP reconstitution observed in the absence and presence of HU treatment, respectively. The results represent an average of 3 to 6 experiments, and the error bars represent the standard error of the mean.

FIG. 6. Dynamic copy-choice model for RT template switching. Shaded boxes represent direct repeats in an RNA template. Horizontal arrows represent nascent DNA. The thickness of these arrows indicate the relative polymerization rate: the thicker the arrow, the faster the rate of polymerization. Small open boxes represent degraded RNA by the RNase H domain. In the case of slow RNase H activity, the degraded RNA fragments are shown as larger open boxes. Hydrogen bonds between the RNA template and nascent DNA are designated by vertical marks. Vertical arrows of various thickness indicate the relative efficiency of template switching. 
Figure 1

A. pES-GFFP

\begin{tabular}{|c|c|c|c|}
\hline \multicolumn{4}{|c|}{ 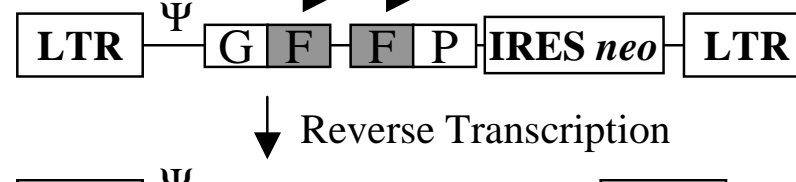 } \\
\hline LTR & \begin{tabular}{|l|l|l|l|l|l|} 
IRES neo & $\mathrm{F}$ & $\mathrm{P}$ \\
\end{tabular} & LTR & \\
\hline LGPS & & & \\
\hline$\Delta$ LTR & pol & $\Delta e n v$ & $\mathbf{p A}$ \\
\hline
\end{tabular}

pSV-A-MLV-env

\begin{tabular}{|l|l|l|l|}
\hline SV40 & SLTR & A-MLV env & pA \\
\hline
\end{tabular}

B. pLGPS

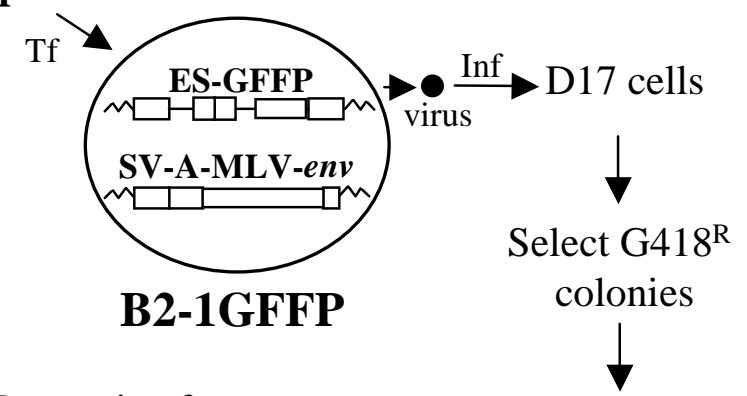

Determine frequency $\longleftarrow$ FACS analysis of GFP reconstitution
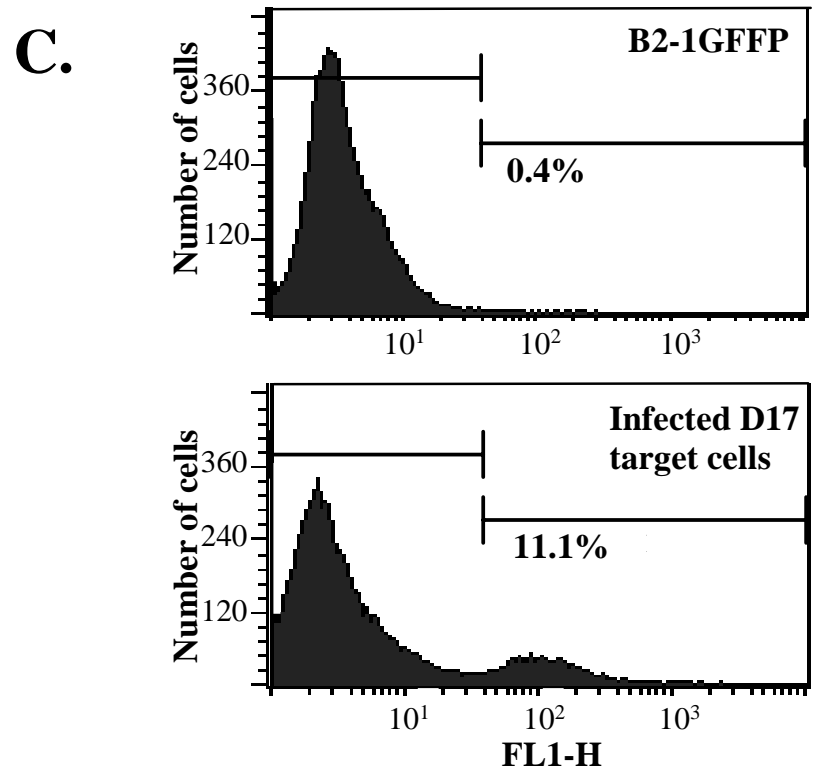
Figure 2
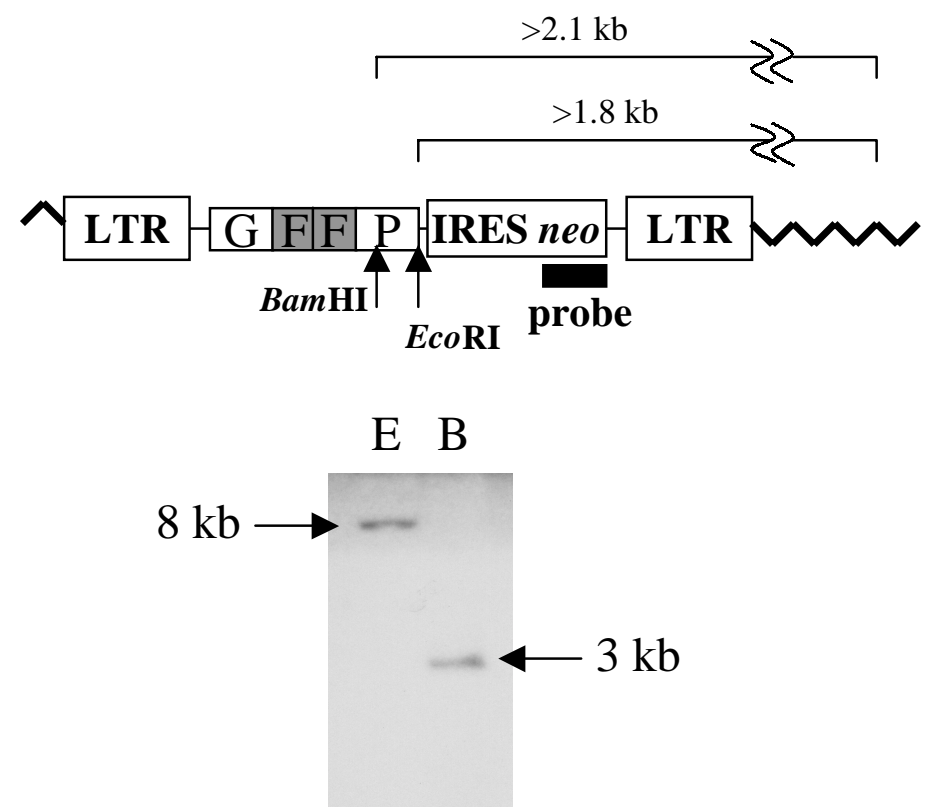


\section{Figure 3}

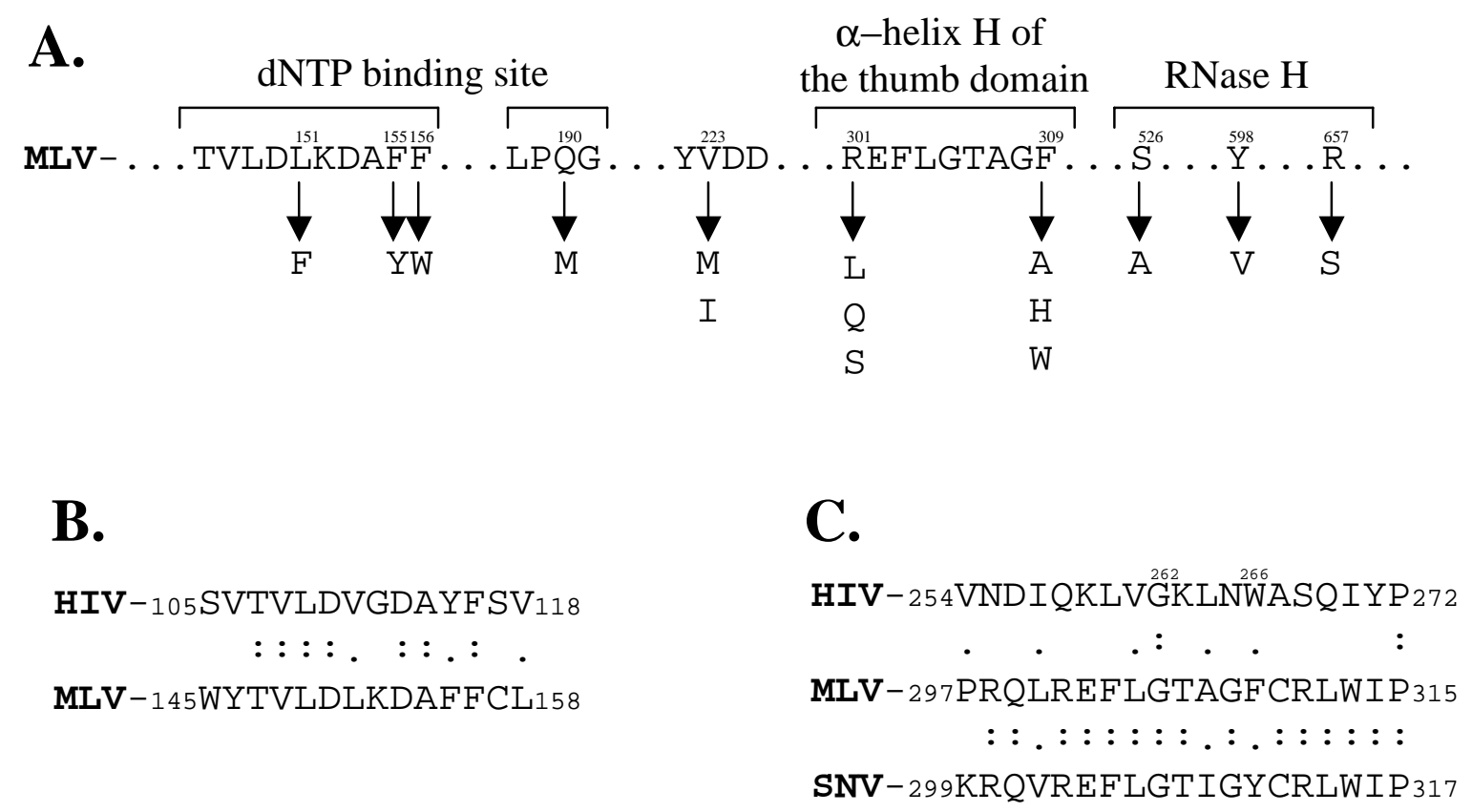


Figure 4

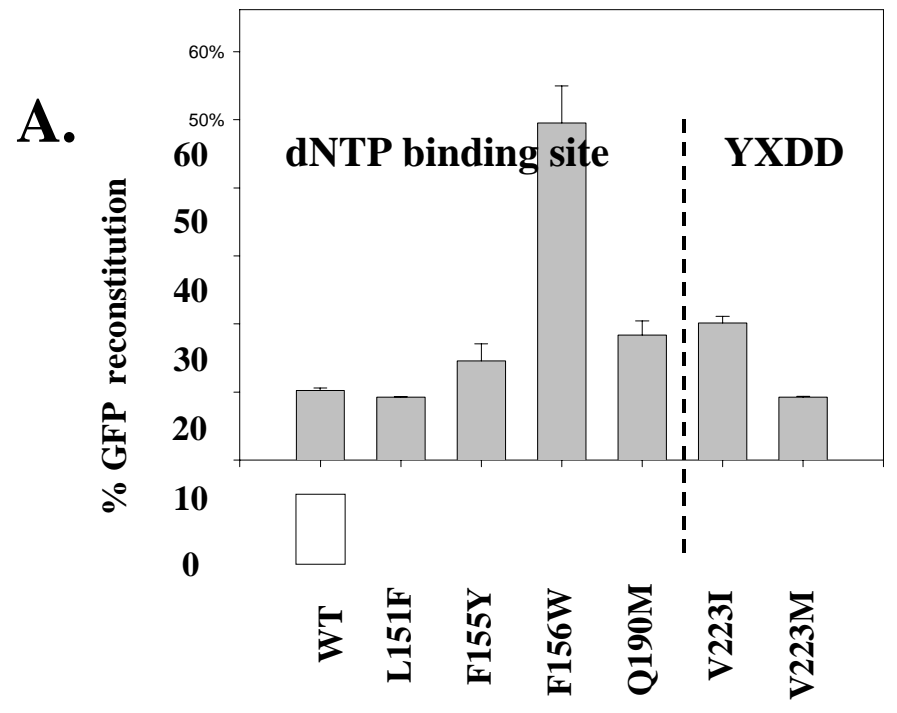

B.

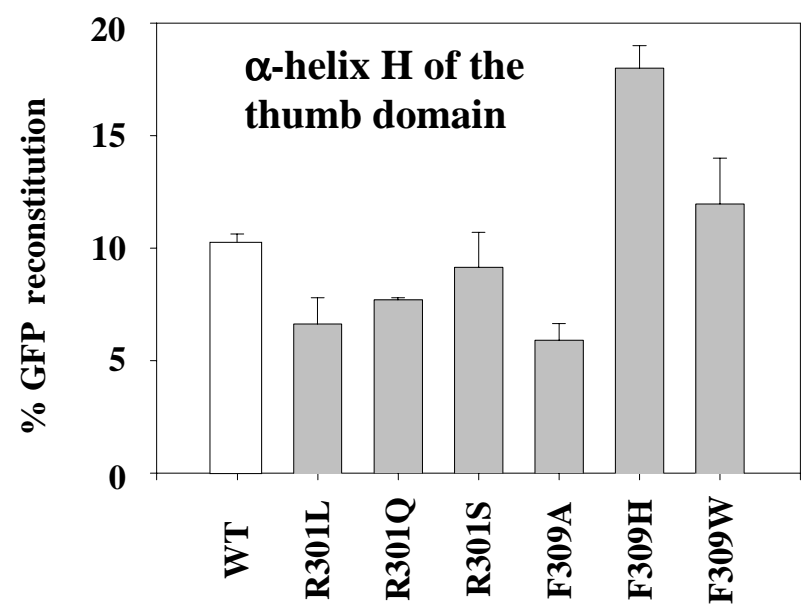

C.

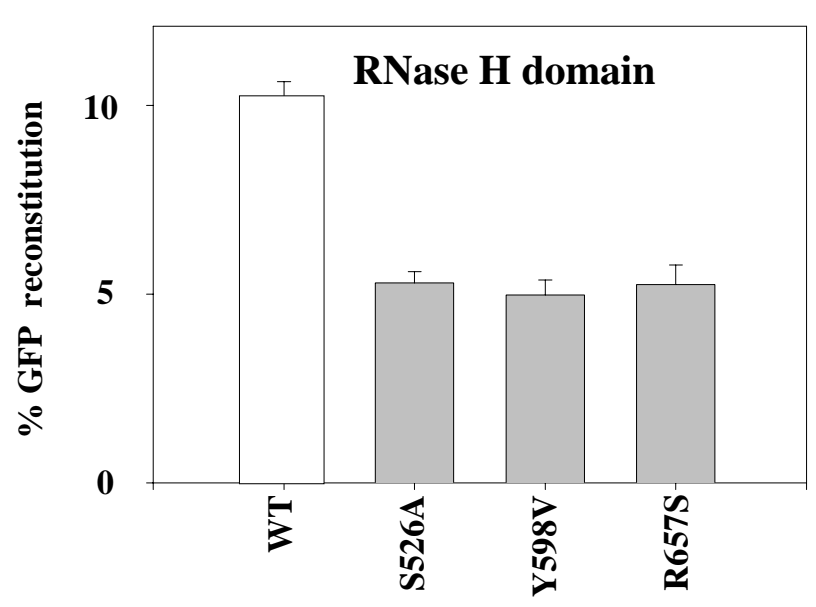


Figure 5

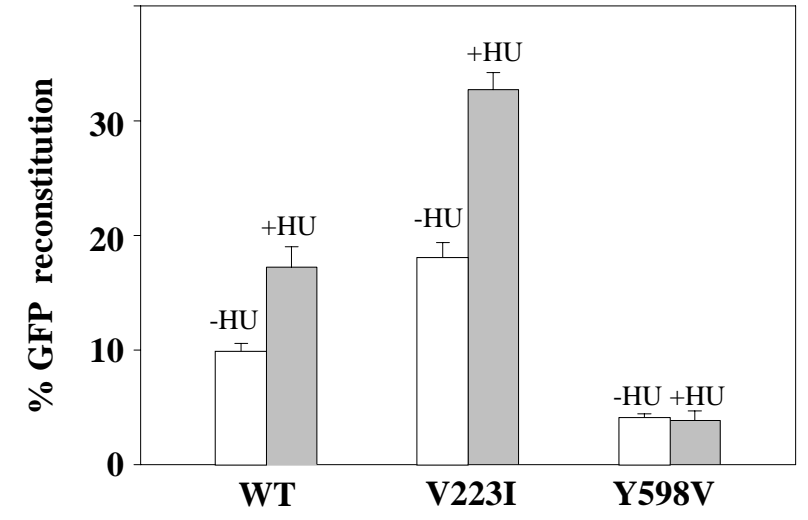


Figure 6

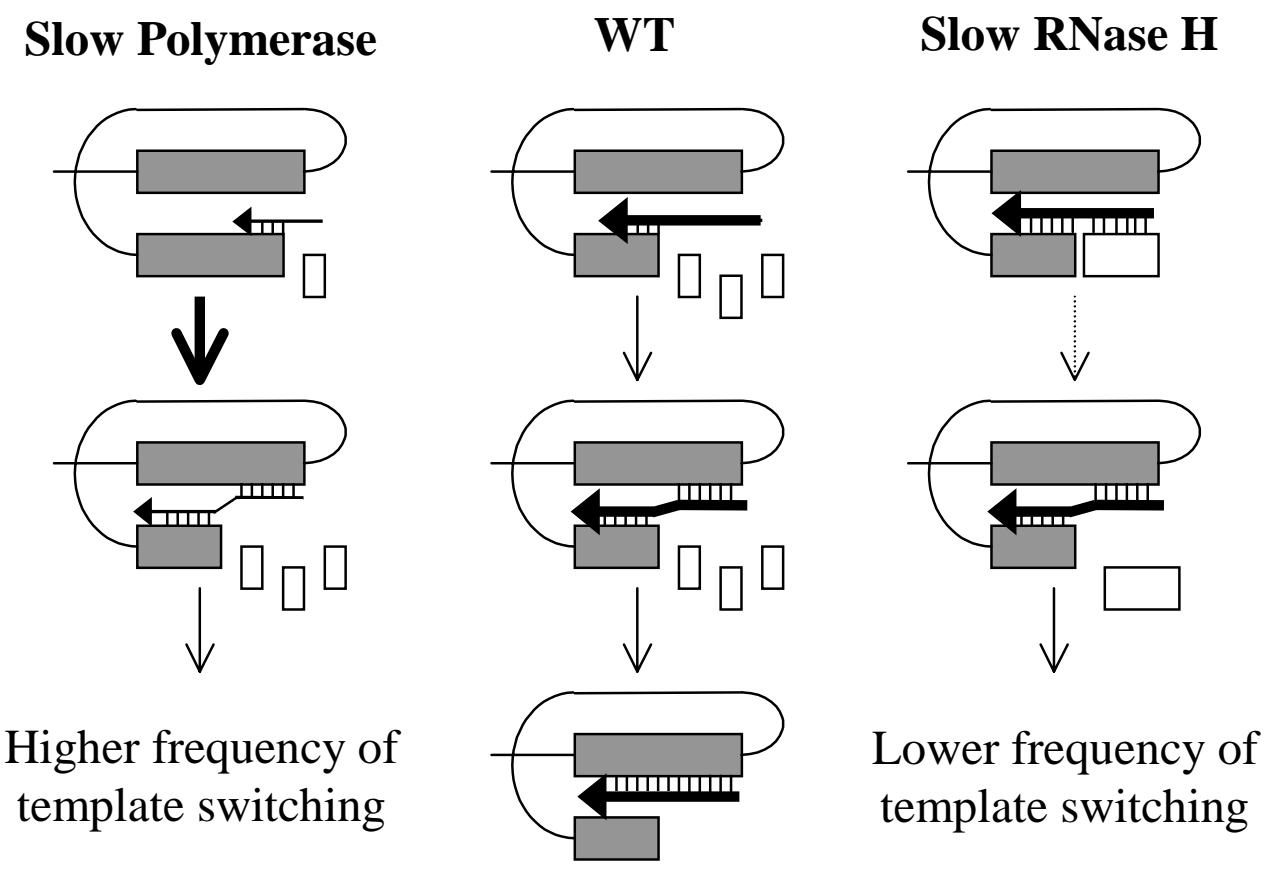




\section{REFERENCES}

1. Bavand, M. R., R. Wagner, and T. J. Richmond. 1993. HIV-1 reverse transcriptase: polymerization properties of the $\mathrm{p} 51$ homodimer compared to the p66/p51 heterodimer. Biochemistry 32:10543-10552.

2. Beard, W. A., K. Bebenek, T. A. Darden, L. Li, R. Prasad, T. A. Kunkel, and S. H. Wilson. 1998. Vertical-scanning mutagenesis of a critical tryptophan in the minor groove binding track of HIV-1 reverse transcriptase. Molecular nature of polymerase-nucleic acid interactions. J. Biol. Chem. 273:30435-30442.

3. Beard, W. A., D. T. Minnick, C. L. Wade, R. Prasad, R. L. Won, A. Kumar, T. A. Kunkel, and S. H. Wilson. 1996. Role of the "helix clamp" in HIV-1 reverse transcriptase catalytic cycling as revealed by alanine-scanning mutagenesis. J. Biol. Chem. 271:12213-12220.

4. Beard, W. A., S. J. Stahl, H. R. Kim, K. Bebenek, A. Kumar, M. P. Strub, S. P. Becerra, T. A. Kunkel, and S. H. Wilson. 1994. Structure/function studies of human immunodeficiency virus type 1 reverse transcriptase. Alanine scanning mutagenesis of an alpha-helix in the thumb subdomain. J. Biol. Chem. 269:28091-28097.

5. Bebenek, K., W. A. Beard, J. R. Casas-Finet, H. R. Kim, T. A. Darden, S. H. Wilson, and T. A. Kunkel. 1995. Reduced frameshift fidelity and processivity of HIV-1 reverse transcriptase mutants containing alanine substitutions in helix $\mathrm{H}$ of the thumb subdomain. J. Biol. Chem. 270:19516-19523. 
6. Blain, S. W., and S. P. Goff. 1995. Effects on DNA synthesis and translocation caused by mutations in the RNase $\mathrm{H}$ domain of Moloney murine leukemia virus reverse transcriptase. J. Virol. 69:4440-4452.

7. Blain, S. W., and S. P. Goff. 1993. Nuclease activities of Moloney murine leukemia virus reverse transcriptase. Mutants with altered substrate specificities. J. Biol. Chem. 268:23585-23592.

8. Bowman, R. R., W. S. Hu, and V. K. Pathak. 1998. Relative rates of retroviral reverse transcriptase template switching during RNA- and DNA-dependent DNA synthesis. J. Virol. 72:5198-5206.

9. Boyer, P. L., and S. H. Hughes. 1995. Analysis of mutations at position 184 in reverse transcriptase of human immunodeficiency virus type 1. Antimicrob. Agents Chemother. 39:1624-1628.

10. Coffin, J. M. 1979. Structure, replication, and recombination of retrovirus genomes: some unifying hypotheses. J. Gen. Virol. 42:1-26.

11. Coffin, J. M., S. H. Hughes, and H. E. Varmus. 1997. Retroviruses. Cold Spring Harbor Laboratory Press, Cold Spring Harbor, NY.

12. Czernilofsky, A. P., A. D. Levinson, H. E. Varmus, J. M. Bishop, E. Tischer, and H. M. Goodman. 1980. Nucleotide sequence of an avian sarcoma virus oncogene (src) and proposed amino acid sequence for gene product. Nature 287:198-203.

13. Delviks, K. A., W. S. Hu, and V. K. Pathak. 1997. Psi- vectors: murine leukemia virus-based self-inactivating and self-activating retroviral vectors. J. Virol. 71:6218-6224. 
14. Delviks, K. A., and V. K. Pathak. 1999. Effect of distance between homologous sequences and 3' homology on the frequency of retroviral reverse transcriptase template switching. J. Virol. 73:7923-7932.

15. Druillennec, S., A. Caneparo, H. de Rocquigny, and B. P. Roques. 1999. Evidence of interactions between the nucleocapsid protein $\mathrm{NCp} 7$ and the reverse transcriptase of HIV-1. J. Biol. Chem. 274:11283-11288.

16. Garces, J., and R. Wittek. 1991. Reverse-transcriptase-associated RNaseH activity mediates template switching during reverse transcription in vitro. Proc. R. Soc. Lond. B. Biol. Sci. 243:235-239.

17. Georgiadis, M. M., S. M. Jessen, C. M. Ogata, A. Telesnitsky, S. P. Goff, and W. A. Hendrickson. 1995. Mechanistic implications from the structure of a catalytic fragment of Moloney murine leukemia virus reverse transcriptase. Structure 3:879-892.

18. Ghosh, M., J. Williams, M. D. Powell, J. G. Levin, and S. F. Le Grice. 1997. Mutating a conserved motif of the HIV-1 reverse transcriptase palm subdomain alters primer utilization. Biochemistry 36:5758-5768.

19. Guo, J., L. E. Henderson, J. Bess, B. Kane, and J. G. Levin. 1997. Human immunodeficiency virus type 1 nucleocapsid protein promotes efficient strand transfer and specific viral DNA synthesis by inhibiting TAR-dependent selfpriming from minus-strand strong-stop DNA. J. Virol. 71:5178-5188.

20. Halvas, E. K., E. S. Svarovskaia, and V. K. Pathak. 2000. Development of an in vivo assay to identify structural determinants in murine leukemia virus reverse transcriptase important for fidelity. J. Virol. 74:312-319. 
21. Halvas, E. K., E. S. Svarovskaia, and V. K. Pathak. 2000. Unpublshed data.

22. Harris, D., P. N. Yadav, and V. N. Pandey. 1998. Loss of polymerase activity due to Tyr to Phe substitution in the YMDD motif of human immunodeficiency virus type-1 reverse transcriptase is compensated by Met to Val substitution within the same motif. Biochemistry 37:9630-9640.

23. Hsiou, Y., J. Ding, K. Das, A. D. Clark, Jr., S. H. Hughes, and E. Arnold. 1996. Structure of unliganded HIV-1 reverse transcriptase at 2.7 A resolution: implications of conformational changes for polymerization and inhibition mechanisms. Structure 4:853-860.

24. Hu, W. S., E. H. Bowman, K. A. Delviks, and V. K. Pathak. 1997. Homologous recombination occurs in a distinct retroviral subpopulation and exhibits high negative interference. J. Virol. 71:6028-6036.

25. Huang, H., R. Chopra, G. L. Verdine, and S. C. Harrison. 1998. Structure of a covalently trapped catalytic complex of HIV-1 reverse transcriptase: implications for drug resistance. Science 282:1669-1675.

26. Hughes, S., and E. Kosik. 1984. Mutagenesis of the region between env and src of the SR-A strain of Rous sarcoma virus for the purpose of constructing helperindependent vectors. Virology 136:89-99.

27. Jang, S. K., M. V. Davies, R. J. Kaufman, and E. Wimmer. 1989. Initiation of protein synthesis by internal entry of ribosomes into the 5 ' nontranslated region of encephalomyocarditis virus RNA in vivo. J. Virol. 63:1651-1660.

28. Jang, S. K., H. G. Krausslich, M. J. Nicklin, G. M. Duke, A. C. Palmenberg, and E. Wimmer. 1988. A segment of the 5' nontranslated region of 
encephalomyocarditis virus RNA directs internal entry of ribosomes during in vitro translation. J. Virol. 62:2636-2643.

29. Ji, X., G. J. Klarmann, and B. D. Preston. 1996. Effect of human immunodeficiency virus type 1 (HIV-1) nucleocapsid protein on HIV-1 reverse transcriptase activity in vitro. Biochemistry 35:132-143.

30. Jin, J., N. Kaushik, K. Singh, and M. J. Modak. 1999. Analysis of the role of glutamine 190 in the catalytic mechanism of murine leukemia virus reverse transcriptase. J. Biol. Chem. 274:20861-20868.

31. Jorgensen, R. A., S. J. Rothstein, and W. S. Reznikoff. 1979. A restriction enzyme cleavage map of Tn5 and location of a region encoding neomycin resistance. Mol. Gen. Genet. 177:65-72.

32. Julias, J. G., D. Hash, and V. K. Pathak. 1995. E- vectors: development of novel self-inactivating and self-activating retroviral vectors for safer gene therapy.

J. Virol. 69:6839-6846.

33. Julias, J. G., T. Kim, G. Arnold, and V. K. Pathak. 1997. The antiretrovirus drug 3'-azido-3'-deoxythymidine increases the retrovirus mutation rate. J. Virol. 71:4254-4263.

34. Julias, J. G., and V. K. Pathak. 1998. Deoxyribonucleoside triphosphate pool imbalances in vivo are associated with an increased retroviral mutation rate. J. Virol. 72:7941-7949.

35. Katz, R. A., and A. M. Skalka. 1990. Generation of diversity in retroviruses. Annu. Rev. Genet. 24:409-445. 
36. Kent, R. B., J. R. Emanuel, Y. Ben Neriah, R. Levenson, and D. E. Housman. 1987. Ouabain resistance conferred by expression of the cDNA for a murine $\mathrm{Na}$, K+-ATPase alpha subunit. Science 237:901-903.

37. Kim, J. K., C. Palaniappan, W. Wu, P. J. Fay, and R. A. Bambara. 1997. Evidence for a unique mechanism of strand transfer from the transactivation response region of HIV-1. J. Biol. Chem. 272:16769-16777.

38. Klarmann, G. J., C. A. Schauber, and B. D. Preston. 1993. Template-directed pausing of DNA synthesis by HIV-1 reverse transcriptase during polymerization of HIV-1 sequences in vitro [published erratum appears in J Biol Chem 1993 Jun 25;268(18):13764]. J. Biol. Chem. 268:9793-9802.

39. Landau, N. R., K. A. Page, and D. R. Littman. 1991. Pseudotyping with human T-cell leukemia virus type I broadens the human immunodeficiency virus host range. J. Virol. 65:162-169.

40. Luo, G. X., and J. Taylor. 1990. Template switching by reverse transcriptase during DNA synthesis. J. Virol. 64:4321-4328.

41. Miller, A. D., J. V. Garcia, N. von Suhr, C. M. Lynch, C. Wilson, and M. V. Eiden. 1991. Construction and properties of retrovirus packaging cells based on gibbon ape leukemia virus. J. Virol. 65:2220-2224.

42. Omer, C. A., K. Pogue-Geile, R. Guntaka, K. A. Staskus, and A. J. Faras. 1983. Involvement of directly repeated sequences in the generation of deletions of the avian sarcoma virus src gene. J. Virol. 47:380-382. 
43. Palaniappan, C., M. Wisniewski, P. S. Jacques, S. F. Le Grice, P. J. Fay, and R. A. Bambara. 1997. Mutations within the primer grip region of HIV-1 reverse transcriptase result in loss of RNase H function. J. Biol. Chem. 272:11157-11164.

44. Pathak, V. K., and W. S. Hu. 1997. "Might as well jump!" Template switching by retroviral reverse transcriptase, defective genome formation, and recombination. Sem. Virol. 8:141-150.

45. Pathak, V. K., and H. M. Temin. 1992. 5-Azacytidine and RNA secondary structure increase the retrovirus mutation rate. J. Virol. 66:3093-3100.

46. Pathak, V. K., and H. M. Temin. 1990. Broad spectrum of in vivo forward mutations, hypermutations, and mutational hotspots in a retroviral shuttle vector after a single replication cycle: deletions and deletions with insertions. Proc. Natl. Acad. Sci. USA 87:6024-6028.

47. Pathak, V. K., and H. M. Temin. 1990. Broad spectrum of in vivo forward mutations, hypermutations, and mutational hotspots in a retroviral shuttle vector after a single replication cycle: substitutions, frameshifts, and hypermutations. Proc. Natl. Acad. Sci. USA 87:6019-6023.

48. Pfeiffer, J. K., R. S. Topping, N. H. Shin, and A. Telesnitsky. 1999. Altering the intracellular environment increases the frequency of tandem repeat deletion during Moloney murine leukemia virus reverse transcription. J. Virol. 73:84418447.

49. Raja, A., and J. J. DeStefano. 1999. Kinetic analysis of the effect of HIV nucleocapsid protein $(\mathrm{NCp})$ on internal strand transfer reactions. Biochemistry 38:5178-5184. 
50. Rodriguez-Rodriguez, L., Z. Tsuchihashi, G. M. Fuentes, R. A. Bambara, and P. J. Fay. 1995. Influence of human immunodeficiency virus nucleocapsid protein on synthesis and strand transfer by the reverse transcriptase in vitro. J. Biol. Chem. 270:15005-15011.

51. Sambrook, J., E. F. Fritsch, and T. Maniatis. 1989. Molecular cloning: a laboratory manual, 2nd ed. Cold Spring Harbor Laboratory Press, Cold Spring Harbor, N.Y.

52. Sarafianos, S. G., V. N. Pandey, N. Kaushik, and M. J. Modak. 1995. Sitedirected mutagenesis of arginine 72 of HIV-1 reverse transcriptase. Catalytic role and inhibitor sensitivity. J. Biol. Chem. 270:19729-19735.

53. Sharma, P. L., and C. S. Crumpacker. 1999. Decreased processivity of human immunodeficiency virus type 1 reverse transcriptase (RT) containing didanosineselected mutation Leu74Val: a comparative analysis of RT variants Leu74Val and lamivudine-selected Met184Val. J. Virol. 73:8448-8456.

54. Smith, C. M., J. S. Smith, and M. J. Roth. 1999. RNase H requirements for the second strand transfer reaction of human immunodeficiency virus type 1 reverse transcription. J. Virol. 73:6573-6581.

55. Suo, Z., and K. A. Johnson. 1998. DNA secondary structure effects on DNA synthesis catalyzed by HIV-1 reverse transcriptase. J. Biol. Chem. 273:2725927267.

56. Suo, Z., and K. A. Johnson. 1997. Effect of RNA secondary structure on the kinetics of DNA synthesis catalyzed by HIV-1 reverse transcriptase. Biochemistry 36:12459-12467. 
57. Suo, Z., and K. A. Johnson. 1997. RNA secondary structure switching during DNA synthesis catalyzed by HIV-1 reverse transcriptase. Biochemistry 36:1477814785.

58. Svarovskaia, E. S., and V. K. Pathak. 2000. Unpublished data.

59. Tamalet, C., J. Izopet, N. Koch, J. Fantini, and N. Yahi. 1998. Stable rearrangements of the beta3-beta4 hairpin loop of HIV-1 reverse transcriptase in plasma viruses from patients receiving combination therapy. AIDS 12:F161-166.

60. Tanese, N., A. Telesnitsky, and S. P. Goff. 1991. Abortive reverse transcription by mutants of Moloney murine leukemia virus deficient in the reverse transcriptase-associated RNase H function. J. Virol. 65:4387-4397.

61. Telesnitsky, A., S. W. Blain, and S. P. Goff. 1992. Defects in Moloney murine leukemia virus replication caused by a reverse transcriptase mutation modeled on the structure of Escherichia coli RNase H. J. Virol. 66:615-622.

62. Telesnitsky, A., and S. P. Goff. 1993. RNase H domain mutations affect the interaction between Moloney murine leukemia virus reverse transcriptase and its primer-template. Proc. Natl. Acad. Sci. USA 90:1276-1280.

63. Temin, H. M. 1993. Retrovirus variation and reverse transcription: abnormal strand transfers result in retrovirus genetic variation. Proc. Natl. Acad. Sci. USA 90:6900-6903.

64. Temin, H. M. 1991. Sex and recombination in retroviruses. Trends Genet. 7:7174. 
65. Tsuchihashi, Z., and P. O. Brown. 1994. DNA strand exchange and selective DNA annealing promoted by the human immunodeficiency virus type 1 nucleocapsid protein. J. Virol. 68:5863-5870.

66. Vara, J. A., A. Portela, J. Ortin, and A. Jimenez. 1986. Expression in mammalian cells of a gene from Streptomyces alboniger conferring puromycin resistance. Nucleic Acids Res. 14:4617-4624.

67. Varela-Echavarria, A., C. M. Prorock, Y. Ron, and J. P. Dougherty. 1993. High rate of genetic rearrangement during replication of a Moloney murine leukemia virus-based vector. J. Virol. 67:6357-6364.

68. Wu, T., J. Guo, J. Bess, L. E. Henderson, and J. G. Levin. 1999. Molecular requirements for human immunodeficiency virus type 1 plus-strand transfer: analysis in reconstituted and endogenous reverse transcription systems. J. Virol. 73:4794-4805.

69. Wu, W., B. M. Blumberg, P. J. Fay, and R. A. Bambara. 1995. Strand transfer mediated by human immunodeficiency virus reverse transcriptase in vitro is promoted by pausing and results in misincorporation. J. Biol. Chem. 270:325332.

70. Zhang, J., and C. M. Sapp. 1999. Recombination between two identical sequences within the same retroviral RNA molecule. J. Virol. 73:5912-5917.

71. Zhang, J., and H. M. Temin. 1994. Retrovirus recombination depends on the length of sequence identity and is not error prone. J. Virol. 68:2409-2414. 


\title{
CHAPTER 3
}

\section{IDENTIFICATION OF MURINE LEUKEMIA VIRUS MINOR GROOVE BINDING HELIX OF THE THUMB DOMAIN AND ITS ROLE IN FIDELITY OF REVERSE TRANSCRIPTION INVIVO}

\author{
EVGUENIA S. SVAROVSKAIA, ${ }^{1,2}$ ELIAS K. HALVAS, ${ }^{1}$ NAGARAJAN \\ PATTABIRAMAN, ${ }^{3}$ and VINAY K. PATHAK ${ }^{2}$ \\ Department of Biochemistry, West Virginia University, Morgantown, West Virginia \\ 26506, ${ }^{1}$ and HIV Drug Resistance Program, ${ }^{2}$ and Advanced Biochemical Computing \\ Center, ${ }^{3}$ National Cancer Institute, FCRDC, Frederick, Maryland 21702.
}

Experiments, described in this chapter, are submitted as a manuscript to Journal of Virology, Aug. 2000. 


\begin{abstract}
Structural determinants of retroviral reverse transcriptases (RTs) that are important for in vivo fidelity are currently being elucidated. We sought to determine whether a minor groove binding helix of the thumb domain and primer grip of murine leukemia virus (MLV) RT are important for in vivo fidelity of reverse transcription. Because the thumb domain of MLV RT has not been crystallized, we utilized homology alignment and molecular modeling to identify residues 297-311 as the MLV RT equivalent of human immunodeficiency virus type1 (HIV-1) minor groove binding helix ( $\alpha$-helix H). Substitution mutations of several residues of the MLV RT minor groove binding helix and the primer grip were generated and their effects on viral replication, RT activity and in vivo fidelity of reverse transcription were determined. We employed a previously described in vivo fidelity assay, which measured the frequency of inactivation of bacterial $\beta$ galactosidase gene in a single cycle of retroviral replication. Mutations in the minor groove binding helix residues R301 and F309 decreased the RT fidelity by up to 2.8 fold. Mutations of residues G305 and W313 resulted in $>$ 10,000-fold reductions in viral titer indicating that these residues are essential for viral replication. Extensive mutational analysis of the MLV RT primer grip residue L269 indicated that 7 of 8 substitutions resulted in $\mathbf{1 0 , 0 0 0 - f o l d ~ r e d u c t i o n s ~ i n ~ v i r a l ~ t i t e r ; ~ t h e ~ L 2 6 9 T ~ m u t a n t ~}$ replicated but did not display any alterations in fidelity of reverse transcription. These results identify the minor groove binding helix of the thumb domain of MLV RT and indicate that it is important for accuracy of viral DNA synthesis.
\end{abstract}




\section{INTRODUCTION}

High evolutionary potential and genetic variation in retroviral populations increases the fitness of the retroviral species. In case of human immunodeficiency virus type-1 (HIV-1), high genetic variation has resulted in the selection of viral variants containing mutations that confer resistance in vivo and in tissue culture experiments to at least 116 antiretroviral drugs (52). The genetic variation in retroviral populations is generated by their high rates of mutations and recombination $(9,23,24,36,37,43-45$, $48,49,56)$. The majority of retroviral mutations are introduced into the viral genome by error-prone reverse transcriptase (RT), a virally encoded enzyme that converts singlestranded viral RNA into double-stranded DNA. The low fidelity of reverse transcription has been well documented by studying mutation rates of RTs in vivo and in vitro $(36,37$, $43-45,48,49)$. Two properties of RT are likely to be responsible for the low fidelity of DNA synthesis during reverse transcription. First, RT lacks a proofreading activity that is present in most cellular polymerases. Second, it has been hypothesized that because two template-switching events are necessary for the completion of reverse transcription, retroviral RTs have evolved to possess low template affinity and low processivity (56). Low template affinity and low processivity may cause additional intramolecular and/or intermolecular template switching events resulting in deletions and recombination.

The structure of RT is likely to play an important role in its low template affinity and low processivity. Several crystal structures of HIV-1 RT have been reported, including co-crystals with non-nucleoside inhibitors, DNA, and a ternary complex with bound DNA and a substrate dTTP $(10,25,33,53)$. HIV-1 RT is a heterodimer composed of $\mathrm{p} 66$ and $\mathrm{p} 51$ subunits. The $\mathrm{p} 66$ subunit resembles a right hand and possesses a 
polymerase and an RNase $\mathrm{H}$ activity. The domains of p66 subunit are referred to as fingers, palm, thumb, connection, and RNase H. The p51 subunit lacks the RNase H domain and folds in a different conformation. The murine leukemia virus (MLV) RT is a monomer in solution and may form a homodimer consisting of two p75 subunits upon interaction with a template-primer (55). A partial MLV RT crystal structure has been solved for an $\mathrm{N}$-terminal segment of the protein containing the fingers and the palm domains $(14,41)$. Despite a low primary sequence homology between HIV-1 and MLV RTs, the three dimensional structures of these proteins are very similar (14).

Several in vitro and in vivo studies have implicated structural determinants of RT that may be important for its fidelity $(1,11,12,17-20,31,38,39,51,57)$. These structural determinants include residues of the Tyr-X-Asp-Asp (YXDD) motif $(1,18,57)$, the deoxyribonucleotide triphosphate (dNTP)-binding site $(12,19,20,38,39,51)$, RNase H domain (18) as well as E89 (11), D76 (31), and F160 (17) in HIV-1 RT.

The thumb domain and the primer grip region are involved in the positioning of the template-primer complex and the affinity of RT to the template-primer. Therefore, these regions may also play an important role in RT fidelity. In the HIV-1 RT, the thumb domain is composed of a bundle of $\alpha$-helices and refers to residues 244-322 (33). It has been proposed that the $\alpha$-helix $\mathrm{H}$ and $\alpha$-helix I form a "helix clamp" and maintain contacts with the template-primer complex during the translocation step of polymerization $(21,22)$. The $\alpha$-helix H of HIV-1 RT is positioned in the minor groove of the template-primer complex (10). Several residues of the $\alpha$-helix $\mathrm{H}$ are part of the motif named as the minor groove binding tract (Q258, G262, W266, Q269, and I94) which makes DNA sequence independent contacts two to six base pairs upstream of the 
$3^{\prime}$ end of the primer $(6,25)$. Alanine-scanning mutagenesis of the $\alpha$-helix $\mathrm{H}$ and $\alpha$-helix I showed that mutations within the $\alpha$-helix $\mathrm{H}$ but not $\alpha$-helix I displayed lower affinity for the template-primer, lower processivity, and frameshift fidelity (2-6). Mutations at the G262 and W266 residues exhibited the most effect on the binding affinity for the template-primer complex, processivity, and fidelity of HIV-1 RT. Additionally, it has been shown that mutations in the thumb domain, especially at the G262 and W266, significantly reduce the amount of full-length DNA product generated, efficiency and specificity of RNase H activity, and efficiency of the strand transfer reaction (13).

Another recent study indicated that substitutions at the $\alpha$-helix $\mathrm{H}$ and $\alpha$-helix I residues result in a loss of cleavage specificity with PPT-containing substrates (46).

The primer grip region is composed a hairpin of two antiparallel $\beta$-sheets of the palm subdomain and consists of highly conserved residues $224-235(25,26)$. It has been postulated that the main function of the primer grip is to position the $3^{\prime}-\mathrm{OH}$ of the primer near to the active site of RT. Several studies have indicated that mutations at the primer grip could affect the binding affinity of RT to the primer and the efficiency with which the RNA primer is extended during minus-strand DNA synthesis $(15,16,27,42,47,59)$. In addition, the primer grip residues are involved in the proper positioning of RT for processing and extension of the polypurine tract (PPT) (47). Recent in vitro data showed that mutations of amino acids at different positions in the primer grip could also affect the misincorporation fidelity of HIV-1 RT (58). Interestingly, some of the mutants were able to improve in vitro RT fidelity. It was hypothesized that the wild type RT has evolved to possess suboptimal fidelity to allow generation of genetic variation (58). 
In this study, we identified a region of MLV RT that is analogous to the minor groove binding helix ( $\alpha$-helix H) of HIV-1 RT. We performed mutational analysis of the minor groove binding helix and primer grip residues to elucidate the role of these regions in fidelity of MLV RT during reverse transcription in vivo. The result showed that proper interactions of RT with primer-template complex are very important for retroviral replication and may affect in vivo fidelity.

\section{MATERIALS AND METHODS}

Plasmids. Plasmid pLGPS expressed the MLV gag and pol genes from a truncated MLV long terminal repeat (LTR) promoter (40). The plasmid pSV-A-MLVenv, which expressed the amphotropic MLV envelope gene from the LTR promoter and SV40 enhancer, was obtained from the AIDS Research and Reference Reagents Program (34). The plasmid pSV $\alpha 3.6$ encoded the $\alpha$ subunit of the murine $\mathrm{Na}^{+}, \mathrm{K}^{+}$-ATPase gene and conferred resistance to ouabain (30). Plasmid pGA-1, a MLV-based retroviral vector, encoded the neomycin phosphotransferase gene (neo) as well as the $\beta$ galactosidase gene (lacZ) (29).

Generation of MLV RT mutants. A detailed description of the mutagenic oligonucleotides and strategies used to generate mutants is available upon request. Briefly, to simplify the mutagenesis procedure, we first constructed pES4, a plasmid analogous to pLGPS that contained only two BamHI sites flanking the primer grip and the minor groove binding helix region. The pES4 was generated by destroying a BamHI site (6601) within pRMBNB, a derivative of pLGPS (19). The destroyed BamHI site was located outside of the gag-pol coding region. PCR-based mutagenesis with random mutagenic primer sets and pES4 as the template was utilized to generate mutants at residues L269, R301, G305, 
F309, L312, and W313. Mutagenic primer sets contained additional silent mutations to generate a restriction site for screening purposes. Amplified DNA fragments of $306 \mathrm{bp}$ were digested with BamHI and subcloned back into pES4. L269 mutants were screened for the presence of a new KpnI site (3797). R301 and G305 mutants were identified by digestion with $E c o$ RI (new site introduced at position 3889). F309 mutants were identified by digestion with PstI (new site introduced at position 3901). L312 and W313 mutants were screened for the presence of a new PstI site (3928). Finally, the mutated plasmids were analyzed by DNA sequencing to verify the presence of the desired mutation and the absence of any undesired mutations (ALF Automated Sequencer, Pharmacia).

Cells, transfections, and infections. D17 dog osteosarcoma cells (obtained from American Type Culture Collection) were transfected, infected, and selected for resistance to ouabain or G418 (a neomycin analog) as previously described $(28,29)$. All cotransfections were performed using a 10:1 molar ratio of the plasmid of interest to the plasmid encoding the selectable marker. D17 and D17-derived cells were maintained in Dulbecco's modified Eagle's medium (DMEM) supplemented with $6 \%$ bovine calf serum, penicillin $(50 \mathrm{U} / \mathrm{ml})$, and streptomycin $(50 \mathrm{mg} / \mathrm{ml})$.

Virus preparation, RT assays, and Western blotting. Virus isolation, concentration, RT assays, and Western blots were performed as previously described (18). Briefly, helper cells containing different pLGPS constructs were plated at $5 \times 10^{6}$ cells per 100-mm dish, $24 \mathrm{~h}$ later media was changed with fresh media containing $1 \%$ calf serum, and an additional 2 days later viruses were collected and centrifuged at 25,000 rpm for $90 \mathrm{~min}$ in an SW41 rotor (Beckman) at $4^{\circ} \mathrm{C}$. Viral pellets were resuspended in phosphate-buffered saline and virus was stored at $-80^{\circ} \mathrm{C}$. 
Exogenous RT activities were determined as previously described using $50 \mu \mathrm{g} / \mu \mathrm{l}$ of (20-mer) oligo (dT) (Integrated DNA Technologies), $100 \mu \mathrm{g} / \mathrm{ml}$ of poly (rA) (Pharmacia), and $10 \mu \mathrm{Ci}$ of $\left[{ }^{3} \mathrm{H}\right]$-dTTP (specific activity of $72 \mathrm{Ci} / \mathrm{mMol}, \mathrm{ICN}$ ). The amount of $\left[{ }^{3} \mathrm{H}\right] \mathrm{dTTP}$ incorporated was determined using a scintillation counter.

Western blots were used to quantify the amount of protein for the RT assays and performed using standard procedures (50). Briefly, viral proteins were resolved using a 13.8\% SDS-PAGE and transferred to membranes (Gelman Sciences, Inc.). Membranes were incubated with primary (monoclonal anti-MLV capsid (CA) IgG1; 1:10 dilution [ATCC]) (8) and secondary antibodies (anti-rat IgG antibody conjugated to horseradish peroxidase; 1:10,000 dilution [Southern Biotechnology Associates, Inc.]). Detection of MLV CA was performed using an ECL (enhanced chemiluminenesence) $\mathrm{kit}^{\mathrm{TM}}$ (Amersham Pharmacia Biotech). The membranes were then exposed to X-OMAT film (Kodak) and the intensity of the p30 band was quantitated using the Image Quant program (Molecular Dynamics).

Protocol for in vivo fidelity assay. The in vivo fidelity assay was described previously (18). Briefly, the ANGIE P cells were plated at a density of $2 \times 10^{5}$ cells per 60-mm-diameter dish, and $24 \mathrm{~h}$ later were cotransfected with wild type or mutated pLGPS and pSV $\alpha 3.6$. The transfected cells were selected for resistance to ouabain $\left(10^{-7}\right.$ M), resistant colonies were pooled, expanded, and plated at a density of $5 \times 10^{6}$ cells per 100-mm-diameter dish. After $48 \mathrm{~h}$, the culture medium containing GA-1 virus was harvested and used to infect D17 target cells plated at a density of $2 \times 10^{5}$ cells per 60mm-diameter dish. Infected D17 cells were selected for resistance to G418 (400 $\mu \mathrm{g} / \mathrm{ml})$ 
and stained with 5-bromo-4-chloro-indolyl- $\beta$-D-galactopyranoside (X-Gal) as previously described (29).

Molecular modeling. The homology modeling tools available in the program Look were used to model the three-dimensional structure of the 267-316 amino acids of the MLV RT (Look version 3.0 is an Integrated Protein Research Tool, Molecular application Group, Palo Alto, CA). The coordinates of crystal structure of a covalently trapped catalytic complex of HIV-1 RT were employed as a structural template for homology model building $(7,25)$.

\section{RESULTS}

\section{Identification of the minor groove binding helix of the MLV RT thumb}

domain. We identified the minor groove binding helix of the MLV RT thumb domain through homology alignment of 40 highly divergent RT sequences (Fig. 1). A simple alignment of MLV RT and HIV-1 RT did not reveal significant homology in the region of the $\alpha$-helix $\mathrm{H}$ and could not be utilized to identify the minor groove binding helix of MLV RT. We first aligned the primer grip, a highly conserved region among all retroviral RTs. The primer grip consists of the following sequence: Y/W/F-L/M-G-X. The $\mathrm{X}$ residue is an aromatic amino acid in 30 of $40 \mathrm{RT}$ sequences. In the remaining RTs, the X residue is a Q (HTLV/BLV viruses and walleye epidermal hyperplasia viruses), L (multiple sclerosis associated virus and cauliflower mosaic virus), or T (mouse mammary tumor virus). We identified a G-X-X-X-W/F/Y motif approximately 30 amino acids downstream of the primer grip in 39 of 40 RT sequences. The only exception was the avian myeloblastosis-associated virus type 1 , which contains a G-X-X$\mathrm{X}-\mathrm{S}$ sequence. The distance between the primer grip and the $\mathrm{G}-\mathrm{X}-\mathrm{X}-\mathrm{X}-\mathrm{W} / \mathrm{F} / \mathrm{Y}$ motif 
was conserved, and varied between 28 to 33 amino acids for retroviral RTs. This distance was slightly longer for nonretroviral RTs (34 and 39 amino acids for cauliflower mosaic virus and hepatitis B virus, respectively). The amino acid G262 through W266 of HIV-1 RT $\alpha$-helix H constitute the G-X-X-X-W/F/Y motif. The sequences of the MLV RT that are adjacent to the $\mathrm{G}-\mathrm{X}-\mathrm{X}-\mathrm{X}-\mathrm{W} / \mathrm{F} / \mathrm{Y}$ motif are highly conserved among the $\mathrm{C}$ type retroviruses, suggesting that they form a structural determinant that is important for RT function. In contrast, the sequences between the primer grip and the G-X-X-X$\mathrm{W} / \mathrm{F} / \mathrm{Y}$ motif are not highly conserved within each group of retroviruses, indicating that RTs can tolerate changes in this region without disruption of RT function. Nevertheless, some amino acids between the primer grip and the $\mathrm{G}-\mathrm{X}-\mathrm{X}-\mathrm{X}-\mathrm{W} / \mathrm{F} / \mathrm{Y}$ motif appeared to be semiconserved in the majority of RT sequences (Fig.1, shaded residues). For example, residues equivalent to the T296 of MLV RT are either a T or an S in 33 of $40 \mathrm{RT}$ sequences. The presence of these semiconserved residues supports the alignment of the G-X-X-X-W/F/Y motif.

It has to be mentioned that MLV RT sequence contains another $\mathrm{G}$ at position 308 that could be aligned with G262 of HIV-1 RT. However, this alignment of G308 of MLV RT with G262 of HIV-1 RT results in positioning L312 of MLV RT in a position of the highly conserved aromatic residue W266 of HIV-1 RT. On the other hand, alignment of the W266 of HIV-1 RT with W313 of MLV RT results in positioning F309 of MLV RT in a position of the highly conserved G262 of HIV-1 RT. Therefore, G308 and W313 of MLV RT are not likely to constitute the G-X-X-X-W/F/Y motif.

Homology modeling of the minor groove binding helix of the MLV RT

thumb domain. To further confirm the identification of the MLV RT minor groove 
binding helix, we built the homology model of the MLV RT amino acids 267 to 316, using the crystal structure of HIV-1 RT bound to the dTTP and DNA as a template (7, 25). The sequence of the homology model included region starting from the primer grip and extended 7 amino acids beyond the G-X-X-X-W/F/Y motif. The homology model was linked at the G270 residue with the crystal structure of fingers and palm domains of MLV RT fragment. The connection between the two fragments was done visually on a computer graphics screen using the modeling program INSIGHT II (BIOSYM/MSI, San Diego, CA) avoiding contacts between these two fragments. A ribbon representation of the superimposed structures of the fingers and palm domains of the MLV RT, modeled thumb domain of MLV RT over the corresponding domains of the HIV-1 RT are shown in Fig. 2A. The magenta colored ribbon shows the homology model of the MLV RT residues 267-316, which include the predicted minor groove binding helix. We were able to build energetically favorable model of the thumb domain of MLV RT using the thumb of HIV-1 RT as template, even though there is not much sequence homology between these two RTs at this region.

The modeled region of MLV RT was four amino acids longer than the homologous sequence of HIV-1 RT. Based on the HIV-1 RT structure, we positioned amino acid Q277 in a loop and amino acids TPK (293-295) in another loop (these loops are indicated by yellow colored ribbon in Fig. 2B). The residues Q277 and TPK (293295) were not modeled, but are presumed to increase the size of the loop without having a substantial effect on the overall structure. The gaps in the sequence alignments in Fig. 1 reflect the view that amino acids equivalent to the MLV RT residues 277 and 293-295 are not present in the HIV-1 RT. 
We analyzed interactions of the identified minor groove binding helix with DNA primer (Fig. 2C). It appears that the G305 and F309 of MLV RT, which constitute the G$\mathrm{X}-\mathrm{X}-\mathrm{X}-\mathrm{W} / \mathrm{F} / \mathrm{Y}$ motif, interact with the primer strand in a manner similar to as G262 and W266 of HIV-1 RT. The $\mathrm{C}^{\alpha}$ atom of the G305 forms van der Waals contact with a sugar ring oxygen atom of the fourth nucleotide upstream of the $3^{\prime}$ end in the primer strand. Residue F309 makes favorable hydrophobic "stacking" interactions with the sugar atoms in the third nucleotide upstream of the $3^{\prime}$ end in the primer strand. The R301 residue does not appear to form contacts with the primer strand and possibly could be exposed to solvent. Additionally, we proposed that R297 and E302 of MLV RT could form saltbridge interactions to stabilize a helical structure of this region (Fig. 2C). D256 and K259 of HIV-1 RT can potentially form similar salt-bridge interactions. Molecular modeling of the MLV RT thumb domain also revealed that Y271, W279, and W313 form hydrophobic interactions, which might be necessary for a proper folding of the thumb domain. Interestingly, these residues are conserved in C-type retroviruses. Similar hydrophobic interactions are formed by Y232, W239, and I270 of HIV-1 RT.

Mutational analysis of the thumb domain of MLV RT. The importance of the identified minor groove binding helix and adjacent residues of the MLV RT for fidelity of reverse transcription was tested using a previously described in vivo assay (18). Briefly, the MLV gag-pol expression construct pLGPS was subjected to site-directed mutagenesis and cotransfected into the ANGIE P cells, which stably express the MLVbased retroviral vector pGA-1 and the MLV envelope expression construct pSV-A-MLVenv (Fig. 3A). Virus harvested from the transfected cell pools was used to infect D17 target cells. The infected D17 cells were selected, stained with X-Gal, and the frequency 
of lacZ inactivation was determined by dividing the number of white colonies by the total number of colonies (blue plus white colonies). The frequency of lacZ inactivation and virus titers provided measures of the in vivo fidelity of reverse transcription and the efficiency of virus replication, respectively (Fig. 3A).

Mutations introduced into the minor groove binding helix of the thumb domain of MLV RT are summarized in Fig. 3B. We mutated amino acid residues R301, G305, and F309 because these residues were expected to contact the template primer and therefore were most likely to affect RT affinity and proper binding to the template-primer. Additionally, mutations at the homologous Q258, G262, and W266 positions of HIV-1 RT displayed the greatest effect on in vitro fidelity, frequency of frameshift mutations and processivity (2-6). We also performed mutational analysis on L312 and W313 because these amino acids are positioned next to the primer grip and are facing the template-primer complex.

The effects of these mutations on in vivo fidelity during reverse transcription were determined and compared to the fidelity of the wild-type MLV RT in parallel experiments (Table 1). Utilization of the wild-type MLV RT consistently resulted in the inactivation of lac $\mathrm{Z}$ with a mean of $5.9 \%$ (Standard error, $\pm 0.4 \%$ ). A total of 7 substitutions were introduced at the R301 position using vertical mutagenesis procedures. Most of the substitutions resulted in significant reductions of viral titers, ranging from 20to greater than 10,000-fold. The only exception was the substitution of R301 with Q, which displayed only a 3 -fold reduction in the viral titer. Notably, a glutamine is present at the equivalent position in the HIV-1 RT as well as 30 of $40 \mathrm{RT}$ sequences analyzed in Fig. 1. Substitution of the R301 with $\mathrm{P}$, which is expected to disrupt $\alpha$-helices, resulted 
in undetectable viral titers. Most of the substitutions resulted in statistically significant 1.5- to 1.9-fold increases in the rates of lac $Z$ inactivation $(P<0.05)$.

All 8 substitutions at the G305 position resulted in undetectable viral titers. This result was consistent with the observation that a glycine is present in all 40 of the RT sequences analyzed in Fig. 1 and indicated that it is very critical for RT function and retroviral replication.

A total of 9 substitutions at the F309 position were analyzed. Substitution of the F309 with a W, which is present at the equivalent position in HIV-1 RT and 25 of the 40 RT sequences analyzed in Fig. 1, displayed the least reduction in viral titer. The F309W displayed a small, but statistically significant increase in fidelity ( 0.7 -fold decrease in frequency of lacZ inactivation, $P<0.05$ ). F309T and F309V did not reduce titers significantly and decreased the accuracy of DNA synthesis. Similar to the R301P, the F309P substitution resulted in undetectable viral titers, reinforcing the view that this region forms an $\alpha$-helix. The rest of the mutations also displayed significant reduction in titers ranging from 33 - to 1,000 -fold. These mutations resulted in an increase in mutation frequencies (1.7- to 2.8-fold). The substitution of F309 with A, a small hydrophobic residue, displayed the most affect on mutant frequency, suggesting that the F309 residue is important for fidelity of DNA synthesis.

Although amino acids L312 and W313 are not conserved among retroviruses, they are present in all C-type retroviruses. Because most of the mutations at L312 and W313 resulted in significant reduction of viral titers $(<10,000$-fold), it was not possible to determine the effect of these mutations on fidelity. However, L312R mutant virus displayed some titer resulting in 82 colonies which we stained with X-Gal and the affect 
of this mutation on the frequency of lac $Z$ inactivation was determined. While the mutation resulted in a two-fold increase in mutant frequency, the number of colonies obtained was insufficient to achieve statistical significance.

DNA polymerization activities of the thumb domain MLV RT mutants. We measured virus associated DNA polymerization activity for the thumb region mutants to determine whether this activity could account for the reduction in viral titers (Fig. 4). Virus was collected from the same number of virus producing cells and pelleted by ultracentrifugation. To normalize the amount of virus used, an aliquot of the viral sample was analyzed by Western blotting using anti-MLV CA antibodies (data not shown). The DNA polymerization activity of the wild type MLV RT was set to $100 \%$ and DNA polymerization activity of each mutant was expressed as a percentage of the wild type in parallel experiments.

The R301Q displayed the highest RT activity as well as the highest viral titer among the R301 mutants tested. With the exception of R301I, the rest of the mutants at this position exhibited low DNA polymerization activities ranging from $8-23 \%$ and displayed viral titers that were lower than $2 \%$ of wild type. The R301I mutant exhibited approximately $30 \%$ of DNA polymerization activity, but the titer of this mutant $(0.5 \%$ of wild type) was lower than titers of the other RT mutants that exhibited lower than $30 \%$ DNA polymerization activities. This suggested that R301I may possess a defect not only in DNA polymerization but also in other functions of RT, such as initiation of minus- or plus-strand synthesis or strand transfer events. The DNA polymerization activities of G305 mutants were generally low, consistent with the low virus titers; however some of the mutants exhibited up to $40 \%$ of DNA polymerization activity (for example G305Q), 
indicating that G305 may play a role in other RT activities mentioned above as well as DNA polymerization. Similar to R301Q, the F309W mutant displayed nearly wild-type DNA polymerization activity. This result was in agreement with the highest titer of F309W mutant among the F309 mutants. Some of the mutants at this position (F309A, F309H, F309I, and F309V) exhibited RT activities higher than wild type and still displayed low titers. These mutants may possess defects in RNase $\mathrm{H}$ activity and result in increased DNA synthesis. We have previously observed that some RNase H mutants exhibit DNA polymerization activities that are grater than wild-type RT (18). It is possible that defects in RNase $\mathrm{H}$ activity reduce the rate of degradation of poly $\mathrm{rA}$ template, which may account for more efficient DNA synthesis in the assay. The DNA polymerization activity of the F309L mutant was nearly 70\%, but the titer of this mutant virus was 1,000-fold lower than the wild type virus titer. The DNA polymerization activities of R301P and F309P were 12\% and 33\%, respectively, suggesting that disruption of the minor groove binding helix of MLV RT thumb domain does not completely eliminate DNA polymerization by the mutant RT. L312E and L312R mutants displayed approximately $40 \%$ and $70 \%$ of wild-type DNA polymerization activity, but possessed very low titers. Low titers of W313 may be explained by a deficiency in other DNA polymerization activities of these mutants. Overall, the DNA polymerization activity data indicates that not all reduction in titers of viruses containing a mutation in the thumb region of MLV RT are due to reductions of DNA polymerization activity of these mutants, suggesting that it is important for other steps of reverse transcription and retroviral replication. 
Structural defect of the MLV RT thumb domain mutants. As indicated above, we performed Western blot analysis for all the mutants to normalize DNA polymerization activity. Western blot of wild type virus with anti-MLV CA antibodies revealed two major bands corresponding to processed CA protein (p30) and unprocessed gag polyprotein (p65) (Fig. 5). Most of the mutants demonstrated a relative ratio of these two bands that was similar to the wild type, indicating that processing of the gag proteins was not altered by mutations in MLV RT (data not shown). Interestingly, the ratio of processed CA protein (p30) and unprocessed gag polyprotein (p65) bands of the R301P, W313A, and W313D mutants were smaller than for wild type, suggesting a processing defect (Fig. 5). This processing defect may be a result of the altered folding of the mutant RTs. The overall RT folding could affect protease folding and as a result decrease processing. Alternatively, mutations in RT may decrease incorporation of the gag-pol proteins into viral particles (possibly because of altered RT folding), providing less protease in viral particles to process gag proteins. However, the unprocessed gag-pol band could be detected in the W313A and W313D mutants, suggesting that it could be incorporated into viral particles (Fig. 5).

Mutational analysis of the primer grip of MLV RT. In addition to the thumb domain mutants, we performed random mutagenesis of L269 of the primer grip in MLV RT. Previous in vitro studies showed that alanine substitution of the analogous M230 in HIV-1 RT displayed higher misincorporation fidelity in comparison to the wild type HIV-1 RT in vitro (58). A total of 8 mutants were tested at the L269 position of MLV RT (Fig. 3). With the exception of L269T, all mutations resulted in undetectable viral 
titers (> 10,000-fold reduction) (Table 2). L269T mutant resulted in reduced titer $(7.5 \%$ of wild type), but did not change the accuracy of DNA synthesis (Table 2).

\section{DISCUSSION}

The importance of the thumb domain of HIV-1 RT has been previously documented. It has been shown that the thumb domain of HIV-1 RT is important for processivity $(2,5,13)$, fidelity in vitro $(2,4,5)$, affinity for template-primer complex $(2$, $4)$, and RNase H cleavage $(13,46)$. It has also been proposed that the thumb domain of HIV-1 RT serves as a "helix clamp" to hold the template-primer complex and as a "protein sensor" of structural alterations in the DNA minor groove upstream of the RT active site $(21,22,35)$. Despite the importance of the thumb domain for RT functions, the thumb domain of other RTs has not been identified or characterized.

In the present study, we identified the minor groove binding helix of the thumb domain of MLV RT based on sequence alignment and in vivo mutational analysis. Sequence alignment of 40 RTs revealed a highly conserved G-X-X-X-W/F/Y motif. This motif points out the position of the minor groove binding helix not only in MLV RT but also in other RTs (Fig.1). This hypothesis was further supported by the fact that amino acids adjacent to this motif are generally conserved within each group of retroviruses and lack the presence of prolines. The aligned sequence also includes the $\alpha$-helix I of HIV-1 RT (277R-Q-L-C-K-L-L283) (25). This sequence is only conserved in three RTs among the 40 compared in this study (HIV-1, HIV-2, and SIV). However, the region which is aligned to the $\alpha$-helix I possesses several semiconserved amino acids P-L-Y-X-X-X-K in other RTs (Fig. 1). The lack of high homology in this region is consistent with a previous mutagenesis study of the $\alpha$-helix I in HIV-1 RT. Mutation of amino acids of the $\alpha$-helix 
I did not affect RT functions as mutations of amino acids of the $\alpha$-helix $\mathrm{H}$ (3). It must to be noted that a different DNA binding motif that is present in HIV-1 RT thumb domain was previously described (22). This motif consists of HIV-1 RT amino acids 259K-L-VG-K-L-(X)16-K-L-L-R284. However, this motif is not conserved in retroviruses and could not be used to define the thumb region.

Further mutational analysis performed in the current study revealed the importance of the minor groove binding helix of MLV RT thumb domain for fidelity of DNA synthesis. This result was in agreement with previous in vitro data for HIV-1 RT. For instance, substitution of the F309 residue of MLV RT with alanine increased the mutation rate by 2.8 -fold (Table 1). The analogous mutation in HIV-1 RT (W266A) resulted in a 3.2-fold increase in the mutation frequency measured by a forward mutation assay in vitro (4). The overall decrease in fidelity correlated with an increase in the rate of frameshift mutations by W266A, W266I, and W266V mutants of HIV-1 RT (2). Mutations of the R301 position of MLV RT that resulted in virus with detectible titer increased mutation frequencies during retroviral DNA synthesis by up to 1.9-fold (Table 1). This observation was consistent with the previously reported 1.9-fold increase in mutation rate in vitro of the R258A mutant of HIV-1 RT (4). The estimation of the mutation frequency of L312R mutant of MLV RT showed a 2-fold increase in comparison to the wild type. Mutation of the same position in HIV-1 RT (Q269A) displayed a similar 2.5-fold increase in mutation frequency in vitro (4).

The results of vertical scanning mutagenesis of the G305 in the current study supported our sequence alignment and indicated that all tested substitutions of the G305 could not be tolerated during retroviral replication. 
Mutational analysis data of the minor groove binding helix of MLV RT also well correlated with the proposed interactions of the this helix and the primer strand (Fig. 2C). It was proposed that residue F309 makes favorable hydrophobic "stacking" interaction with the sugar atoms of nucleotides in the primer strand. Mutation F309W retains the same interaction with the primer strand. However, F309T and F309V provide some hydrophobic interaction with the sugar in the primer strand and therefore these mutant viruses retain considerable viral titers. Mutation F309A contains also a hydrophobic side chain, however it is too small to provide necessary interactions. The F309H, F309I, F309L and F309N mutations resulted in the server steric clash between side chain atoms and the primer strand and therefore result in very low viral titers.

The G305 form the van der Waals contact with the sugar ring oxygen atom of the nucleotide in the primer strand. It is conceivable that even the smallest amino acid substitution at the G305 position tested in this study (G305V) will push the minor groove binding helix away from the primer by at least 2 angstroms and disrupt interactions of the DNA primer not only with G305 (G262 in HIV-1 RT), but also with F309 (W266 in HIV-1 RT). Previous data indicated that disruption of these interactions by mutations at G262 and W266 residues significantly decreased the processivity of $\operatorname{HIV}-1$ RT $(2,5,13)$. Additionally, mutants of G262 and W266 positions have been shown to have a defect in strand-transfer reactions and specificity of the PPT cleavage $(13,46)$.

Recently, we developed an in vivo assay to study the effect of mutations in MLV RT on template switching frequency during reverse transcription (54). In that study we tested several thumb domain mutants of MLV RT. The results indicated that R301Q, R301S, and F309W did not affect the frequency of template switching. Again, it was 
consistent that substitutions to amino acids which are present in HIV-1 RT at the same positions, did not change the function of RT. The R301L and F309A mutants decreased the ability of MLV RT to switch templates during reverse transcription. The proposed dynamic copy-choice model suggested that RNase $\mathrm{H}$ activity is one of the factors in determining the frequency of template switching. Perhaps, R301L and F309A possess a deficiency in RNase $\mathrm{H}$ activity and as a result decrease template switching frequency. The R309H mutant displayed an increase in template switching frequency. For this particular mutant it is possible that the RNase $\mathrm{H}$ activity is not affected to the same extent as for R301L and F309A, but template affinity might be decreased resulting in more frequent template switching events.

Vertical scanning mutagenesis of L269 in MLV RT primer grip presented in this study showed that almost any substitutions of this amino acid resulted in noninfectious virus. These data is in agreement with two observations. First, only leucine or metionine residues are present at this position in all 40 RTs analyzed in Fig. 1. Second, despite the fact that F227, W229 and L234 of the HIV-1 RT primer grip are part of the binding pocket for non-nucleoside RT inhibitors, no drug resistant mutations were detected in the primer grip region (53). Recent in vitro data indicated that mutations of the primer grip in HIV-1 RT, including M230A, could increase misinsertion fidelity in vitro (58). The L269A, a similar mutant in MLV RT tested in this study, did not display any viral titer (Table 2).

\section{ACKNOWLEDGMENTS}

We especially thank Wei-Shau Hu for valuable intellectual input and discussions throughout this project. We thank Benjamin Beasley, Sara Cheslock, Que Dang, Krista 
Delviks, Willam Fu, Carey Hwang, Timur Kabdulov, Dexter Poon, Terence Rhodes, Rebekah Thorick, Yegor Voronin, and Wen-Hui Zhang for their critical reading of this manuscript and discussion of results. We also thank William McBee and April Cooper for their technical support in generating minor groove binding helix mutants. Finally, we extend our thanks to Anne Arthur for her editorial expertise and revisions. 
Table 1. Effects of mutations of the MLV RT thumb region on the frequency of lacZ inactivation.

\begin{tabular}{|c|c|c|c|c|c|}
\hline $\begin{array}{l}\text { MLV RT } \\
\text { Genotype }\end{array}$ & $\begin{array}{l}\text { No. } \\
\text { of } \\
\text { Expts. }\end{array}$ & $\begin{array}{c}\text { No. Mutant } \\
\text { Colonies/ } \\
\text { Total Colonies }\end{array}$ & $\begin{array}{c}\text { Frequency } \\
\text { of lacZ } \\
\text { Inactivation } \\
(\% \text { S.E. })^{\mathrm{b}}\end{array}$ & $\begin{array}{l}\text { Relative } \\
\text { Change in } \\
\text { Inactivation } \\
\text { of } l a c Z^{\mathrm{c}}\end{array}$ & $\begin{array}{l}\text { Relative } \\
\text { Viral } \\
\text { Titer }^{\mathrm{d}}\end{array}$ \\
\hline WT & 7 & $227 / 3,953$ & $5.9 \pm 0.4$ & 1.0 & 1.00 \\
\hline $\mathrm{R} 301 \mathrm{H}$ & 2 & $79 / 785$ & $9.5 \pm 0.1$ & 1.6 & 0.02 \\
\hline R301I & 2 & $44 / 382$ & $11.5 \pm 0.5$ & 1.9 & 0.005 \\
\hline R301L & 2 & $96 / 1,536$ & $6.6 \pm 0.9$ & none & 0.02 \\
\hline R301P & 1 & $\mathrm{NC}^{\mathrm{e}}$ & $N A^{f}$ & NA & $<0.0001$ \\
\hline R301Q & 2 & $216 / 2,430$ & $8.8 \pm 0.5$ & 1.5 & 0.30 \\
\hline R301S & 2 & $141 / 1,696$ & $9.1 \pm 2.1$ & 1.5 & 0.05 \\
\hline R301V & 2 & $101 / 910$ & $9.8 \pm 2.1$ & 1.7 & 0.01 \\
\hline $\begin{array}{l}\text { G305 D, E, F, } \\
\text { I, N, Q, R, V }\end{array}$ & 1 & $\mathrm{NC}$ & NA & NA & $<0.0001$ \\
\hline F309A & 2 & $260 / 1,802$ & $13.7 \pm 0.3$ & 2.8 & 0.02 \\
\hline $\mathrm{F} 309 \mathrm{H}$ & 2 & $40 / 780$ & $5.3 \pm 0.3$ & none & 0.03 \\
\hline F309I & 2 & $34 / 366$ & $9.1 \pm 0.2$ & 1.7 & 0.07 \\
\hline F309L & 3 & $\mathrm{NC}$ & NA & NA & $<0.001$ \\
\hline F309N & 2 & $65 / 490$ & $13.3 \pm 0.9$ & 2.3 & 0.02 \\
\hline F309P & 1 & $\mathrm{NC}$ & NA & NA & $<0.0001$ \\
\hline F309T & 2 & $109 / 1,073$ & $9.3 \pm 1.1$ & 1.6 & 0.13 \\
\hline F309V & 2 & $36 / 449$ & $7.7 \pm 0.7$ & 1.3 & 0.12 \\
\hline F309W & 2 & $27 / 693$ & $3.9 \pm 0.1$ & 0.7 & 0.18 \\
\hline L312E & 1 & $\mathrm{NC}$ & NA & NA & $<0.0001$ \\
\hline L312R & 2 & $8 / 82$ & 9.7 & NA & $<0.001$ \\
\hline W313 A, D, Q & 1 & $\mathrm{NC}$ & NA & NA & $<0.0001$ \\
\hline
\end{tabular}


a The number of mutant colonies that displayed a white colony phenotype and the total number of colonies that were observed in 1 to 7 independent experiments.

${ }^{\mathrm{b}}$ The frequency of lacZ inactivation was calculated as follows: No. of mutant colonies in each experiment $\div$ by total number of colonies $\times 100 \%$. The standard error of the mean (SE) was determined by using the Sigma Plot 5.0 program.

${ }^{c}$ The relative change in the frequency at which the lac $Z$ gene was inactivated was calculated as follows: frequency of lacZ inactivation observed with mutant MLV RT $\div$ by frequency of lacZ inactivation observed with wild type MLV RT in the same experiment. Statistical analysis using a Two-sample T-Test showed that R301H, R301I, R301Q, R301S, R301V, F309A, F309I, F309N, F309T, F309V, F309W mutants of MLV RT displayed a significantly different mutant frequency in comparison to the wild type MLV RT $(P<0.05)$. The mutant frequency obtained with the R301L and F309H mutants of MLV RT was not significantly different from the wild type MLV RT $(P>0.05)$.

${ }^{\mathrm{d}}$ Virus titer for each experimental group was determined by serial dilutions. The average virus titer obtained with wild type MLV RT was $3.1 \times 10^{4} \mathrm{CFU} / \mathrm{ml}$. The relative virus titer represents a ratio of the virus titer of mutant MLV RT obtained in each experiment divided by the virus titer obtained with wild type MLV RT in parallel experiments.

${ }^{\mathrm{e}} \mathrm{NC}$ represents no colonies present after G418 selection signifying titers less than $10,000 \mathrm{CFU} / \mathrm{ml}$.

${ }^{\mathrm{f}} \mathrm{NA}$ represents not applicable since the relative change in the inactivation of lac $Z$ gene could not be determined due to the absence or low number of colonies after G418 selection. 
Table 2. Effects of mutations of the primer grip of MLV RT on the frequency of lacZ inactivation

\begin{tabular}{|c|c|c|c|c|c|}
\hline $\begin{array}{l}\text { MLV RT } \\
\text { Genotype }\end{array}$ & $\begin{array}{l}\text { No. } \\
\text { of } \\
\text { Expts. }\end{array}$ & $\begin{array}{c}\text { No. Mutant } \\
\text { Colonies/ } \\
\text { Total Colonies }\end{array}$ & $\begin{array}{c}\text { Frequency } \\
\text { of lacZ } \\
\text { Inactivation } \\
(\% \text { S.E. })^{b}\end{array}$ & $\begin{array}{l}\text { Relative } \\
\text { Change in } \\
\text { Inactivation } \\
\text { of lac } Z^{c}\end{array}$ & $\begin{array}{l}\text { Relative } \\
\text { Viral } \\
\text { Titer }^{\mathrm{d}}\end{array}$ \\
\hline WT & 2 & $50 / 1096$ & $4.4 \pm 0.4$ & 1.0 & 1.00 \\
\hline L269T & 2 & $53 / 904$ & $6.3 \pm 0.9$ & none & 0.075 \\
\hline $\begin{array}{c}\mathrm{L} 269 \mathrm{~A}, \mathrm{G}, \mathrm{H}, \\
\mathrm{N}, \mathrm{Q}, \mathrm{R}, \mathrm{S}\end{array}$ & 1 & $\mathrm{NC}^{\mathrm{e}}$ & $N A^{f}$ & NA & $<0.0001$ \\
\hline
\end{tabular}

${ }^{a}$ The number of mutant colonies that displayed a white colony phenotype and the total number of colonies that were observed in 2 independent experiments.

${ }^{\mathrm{b}}$ The frequency of lacZ inactivation was calculated as for table1.

${ }^{\mathrm{c}}$ The relative change in the frequency at which the lacZ gene was inactivated was calculated as for table 1. Statistical analysis using a Two-sample T-Test showed that L269T mutant of MLV RT did not display a mutant frequency significantly different from the wild type MLV RT $(P=0.199)$.

${ }^{\mathrm{d}}$ Virus titer for each experimental group was determined by serial dilutions. The average virus titer obtained with wild type MLV RT was $1.5 \times 10^{4} \mathrm{CFU} / \mathrm{ml}$. The relative virus titer represents a ratio of the virus titer of mutant MLV RT obtained in each experiment divided by the virus titer obtained with wild type MLV RT in parallel experiments.

${ }^{\mathrm{e}} \mathrm{NC}$ represents no colonies present after G418 selection signifying titers less than $10,000 \mathrm{CFU} / \mathrm{ml}$.

${ }^{\mathrm{f}} \mathrm{NA}$ represents not applicable since the relative change in the inactivation of lacZ gene could not be determined due to the absence of colonies after G418 selection. 


\section{FIGURE LEGENDS}

\section{FIG. 1. Amino acid sequence alignment of primer grip and thumb domain}

region of retroviral reverse transcriptases. Black shading indicates primer grip region and conserved G-X-X-X-W/F/Y (aromatic amino acid) motif of the minor groove binding helix of the thumb domain. Gray shading indicates conserved and semiconserved amino acids that are present in MLV RT. An open box designates the $\alpha$ helix H of HIV-1 RT based on crystal structure data (25). The underlined amino acids are conserved or semiconserved between MLV RT and HIV-1 RT. The accession number is a number assigned to each sequence in the protein database. G305 and F309 of MLV RT and G262 and W266 of HIV-1 RT are labeled by correspondent numbers.

FIG. 2. Model of the MLV RT Thumb domain. (A) A ribbon representation of the superimposed structure fingers and thumb domain of the MLV RT over the corresponding domain of the HIV-1 RT. The white ribbon represents the HIV-1 RT. The yellow ribbon represents the crystal structure of MLV RT. The magenta colored ribbon shows the homology model of the MLV RT thumb region. Residues 16 to 22 of the primer strand are shown as cyan bonds. (B) The primer binding pocket of HIV-1 RT (25). Residues that were used for homology modeling study is shown by ribbon representation. The rest of the residues in the primer-binding pocket is shown as c-alpha atom trace. Residues 16 to 22 of the primer strand are shown as cyan bonds. Yellow colored ribbon represents loops where amino acid Q277 in a loop and amino acids TPK (293-295) of MLV RT were positioned. (C) Interactions of the minor groove binding helix of MLV RT with the primer. Minor groove binding helix of MLV RT is shown as a ribbon representation. The primer nucleotides are shown as atom-based color-coded 
bonds. The residues, R297, R301, E302, G305 and F309 are labeled. The c-alpha atom of G305, the side chain atoms of F309, and oxygen atom of the fourth nucleotide upstream of the 3' end in the primer strand, and the sugar ring atoms of the third nucleotide of the 3' end in the primer strand are shown as van der Waals spheres.

FIG. 3. Mutational analysis of the minor groove binding helix of MLV RT. (A) Protocol for in vivo assay to identify structural determinants of MLV RT important for fidelity. The pLGPS construct expresses the MLV gag and pol from a truncated viral LTR. The wild-type or mutated pLGPS constructs were separately cotransfected (Tf) with pSV 23.6 into the ANGIE P cells. ANGIE P, a D17-based cell line, expressed pGA1 and pSV-A-MLV-env. The pGA-1 vector contains LTRs and all cis-acting elements of MLV. The pGA- 1 also encodes for $\beta$-galactosidase reporter gene (lacZ). The lacZ and neo are transcribed from the LTR promoter and IRES of encephalomyocardidtis virus is used to express neo. $\Psi$ designates MLV packaging signal. The pSV-A-MLV-env construct expresses the amphotropic MLV envelope from a truncated MLV LTR and the SV40 promoter enhancer. The virus produced was used to infect D17 cells. The infected cell clones resistant to G418 were stained with X-Gal and frequencies of inactivation of lacZ reporter gene were determined. (B) Mutants of the primer grip and thumb domain of MLV RT. Primary sequence containing the primer grip and the minor groove binding helix of the thumb domain is shown. The numbers above the primary sequence indicate the amino acid positions in the primary sequence. The substitution mutations analyzed at each amino acid position are indicated below the primary sequence with downward arrows. 
FIG. 4. RT activities for virion-associated RTs. The activities shown are relative to the RT activity determined for the wild-type MLV RT (set 100\%). The results represent an average of two to three independent experiments. The error bars represent the standard error of the mean.

FIG. 5. Decreased processing of mutant viruses. A representative Western blot analysis is shown for the wild-type (WT) virus and viruses containing mutation in RT (R301P, W313A, and W313D). Unprocessed gag-pol, unprocessed gag (p65) and processed CA (p30) bands are indicated by arrows. 
Figure 1

C-Type Retroviruses

Moloney murine leukemia virus

Gibbon leukemia virus

Phascolarctos cinereus (Koala)

Porcine endogenous retrovirus

Baboon endogenous virus

Feline leukemia virus

Spleen necrosis virus

\section{Lentivir}

Human immunodeficiency virus type 1

Human immunodeficiency virus type 2

Simian immunodeficiency virus

Puma lentivirus 14

Visna virus

Ovine lentivirus

Jembrana disease virus

Caprine arthritis-encephalitis virus

Feline immunodeficiency virus

Equine infectious anemia virus

Human T-cell lymphotropic virus type Human T-cell lymphotropic virus type

Simian T-cell lymphotropic virus type 2

Simian T-cell lymphotropic virus

Bovine leukemia virus

Bovine syncytial virus

Simian foamy virus type 3

\section{D-Type Retroviruses}

Mason-Pfizer monkey virus

Simian SRV-2 type D retrovirus

Simian type D virus 1

Ovine pulmonary adenocarcinoma virus

Simian sarcoma virus

\section{Avian and other retroviruses}

Multiple sclerosis assoc. retrovirus

Walleye epi. Hyp. virus type

Walleye epi. Hyp. virus type

Rous sarcoma virus

Avian leukosis virus

Avian myeloblastosis virus

Lymphoproliferative disease virus

Mouse mammary tumor virus

Nonretroviral RTs

Hepatitis B virus

Cauliflower mosaic virus

Grip

28-39a.a. GXXXW/F/Y motif

accession no. AAC82568.1 AAC80264.1 AAF15098.1 CAB 65341.1 BAA89659.1 AAA93092.1 unpublished

Ref. 26

AAA 64576.1 AAA 74707.1 AAA 67168.1 AAA 17524.1 AAA 66821.1 AAA 64389.1 AAA91826.1 AAA 4 3071.1 AAA43004.1

AAA 85843.1 AAB59885.1 AAB 58786.1 CAA68894.1 AAC 82587.1 AAB 68770.1 AAA 47796.1

AAA 47711.1 AAA 4 7562.1 AAC 97565.1 AAD 45226.1 AAA 66453.1

AAB 66528.1 AAC59311.1 AAC59310.1 CAA48535.1 AAA 91269.1 AAB31929.1 AAA 62195.1 BAA0 3767.1 1 AAA 46304. AA69721 FMGYV Q02964

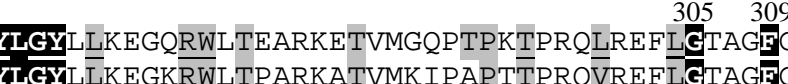
GYLLKGGKRWLTPARKATVMKIP TP TTPRQVREF GYSLRGGQRWLTEARKKTVVQIPAPTTAKQVREF ILSEGKRWLTP GRIETVARIPPPRNPREVREF SLKDGQRWLTKARKEA I LS IPVPKNPRQVRE KIHKGSRTLSNSRTQA I LQIPVPKTKRQVREFI HPD-KWTVQP IVLPEKDSW---TVNDIQKL ELWP T-KWKLQKIQLP QKEVW---TVND IQKL TLHPD-KWQLQKIELPNIDDEI--TVNQLQKLI LYPN-KWT IQKTKLD IPEVP---TLNQLQKLA HPE-KWKFOKHTLPEITEGP I-TLNKLOK LHPE-KWRFQKHTLPEIKEGTI-TLNKLQKL LLTPK-RWRFQPRQIKIKKVV---TVNELQQM ELHPQ-TWKF QKHTLPELTKGTI-TLNKLQKLV ELHP L-TWS IQQKQLE IP ERP---TLNELQKLA YQLCP E-NWKVQKMQLDMVKNP---TLNDVQKLMGNI TWMS SGVP GLTVKH IAATTKGC

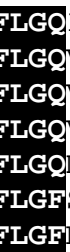

ISPN--HLTYDAVPTVP IRSRW-ALPELQAL ISPN--HITYESTPTIP IKSOW-TLTELOV ISPD--HITYETTPTIPMKSQW-TLAELQTVL ISQD--CITYETLPSIHIKSTW-SLAELQSML VHNQ--IVTYQSLPTLQISSP I-SLHQLQAV I SONGRGLTDSYKOKLMDLOPPTTLROLOS I ITKEGRGLTETFKQKLLNITPPRDLKQLQS I

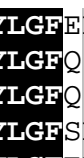

LNGP-KITNOKAVIRKDKLO---TLNDFOKL NGP-KITNOKAVIRRDKLO---TLNDFOK INGP-KI INQKAVIRHDHLK---TLNDFQKLI YPR-VYNTOLVKLOTDHLK---TLNDFOKL LHHQQVF-TPRVCLKTDHLK---TLNDFQKI

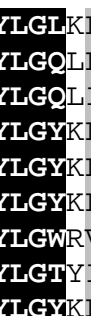

SKGTRALSEERIQP ILAYPHPKTLKQLRGF SDRGRE I LPDRKETVSQF SSP T TVRQVRAF SKAGRD I LPERKKTVSQFAAPTTVRQVRA LGST--YVAPVGLVAEPRIA---TLWDVQKLV
T LGST--YVAPVGLVAEPRIA---TLWDVQKLV GST--YVAPVGLVAEPRIA---TLWDVQK YIOGD--VVSYOKLOIRTDKLR--TLNDFOKL GYKLGST--YVAPVGLVAEPRIA---TLWDVQKL

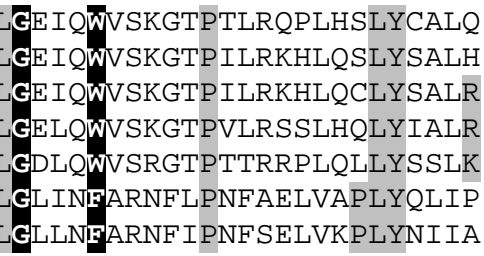

ARNF IPNF SELVKP LYNI IA

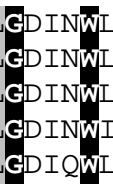

RPYLKLTTGDLKPLFDTLK RPYLHLTTGDLKPLFDILK RPYLKLTTGELKPLFDILK RPYLKLPTYTLQP LED ILK RPYLKLPTSALVP LNNILK

GSWGTLPQEH IVLKIKQCFRKLPVNRP IDWKVCQRIV

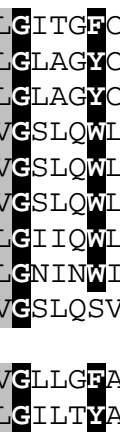

CRKQIPRYTPIARPLYTLIR RHWIPDYSEQSKYLEELLK RHWIADYSENSKHLEELLK RPALGIPPRLMGPFYEQLR RPALGIPPRLMGPFYEQLR RPALGIPPRLRGPFYEQLR KP I LS LRPEQLQVFYDLLK IRPFLKLTTGELKPLFEILN VRPALGIPPRLMGPFYEQLR

APFTQCGYPALMPLYACIQ LGLE IDEGTHKP QGH I LEH INKF P D T LEDKKQLQRFL LI L TYASDY IPKLAQ I RKP LQAKLK 
Figure 2
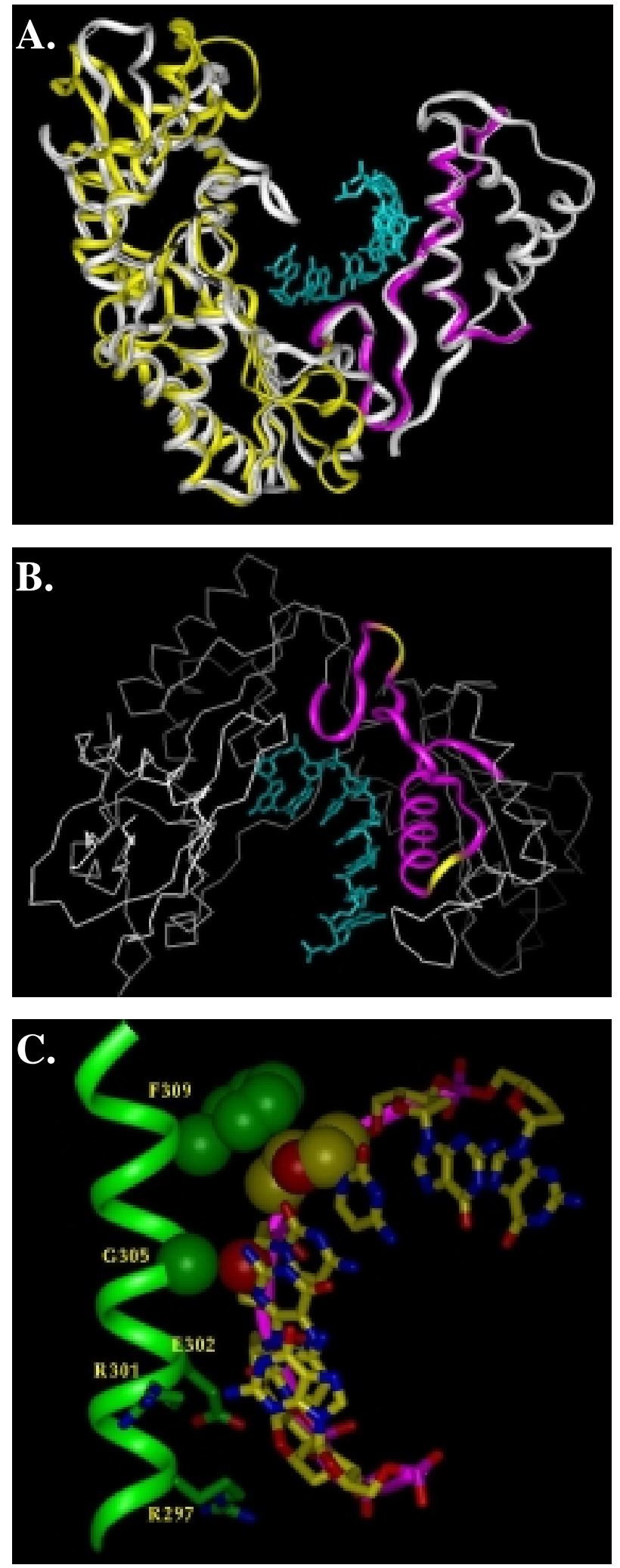
Figure 3
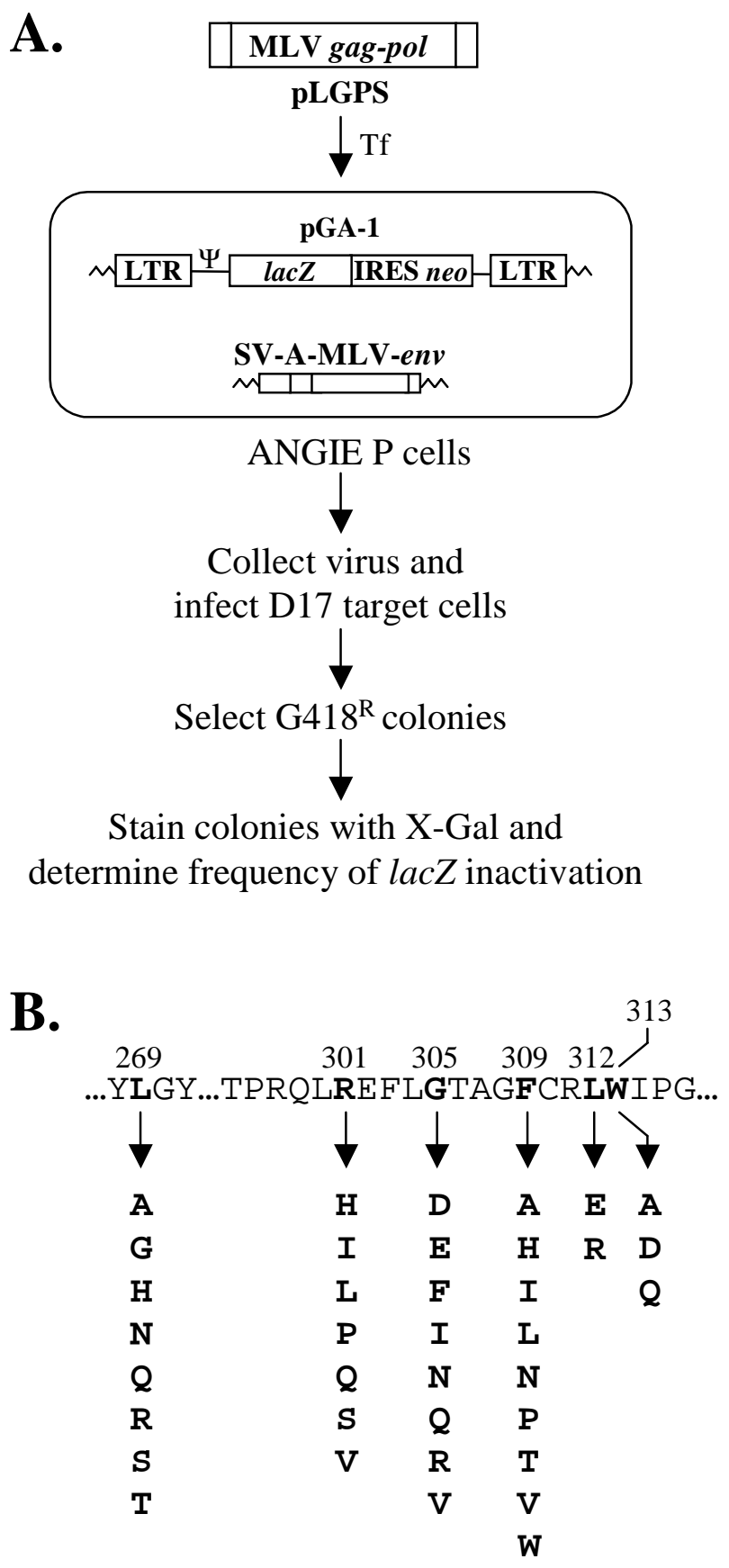
Figure 4
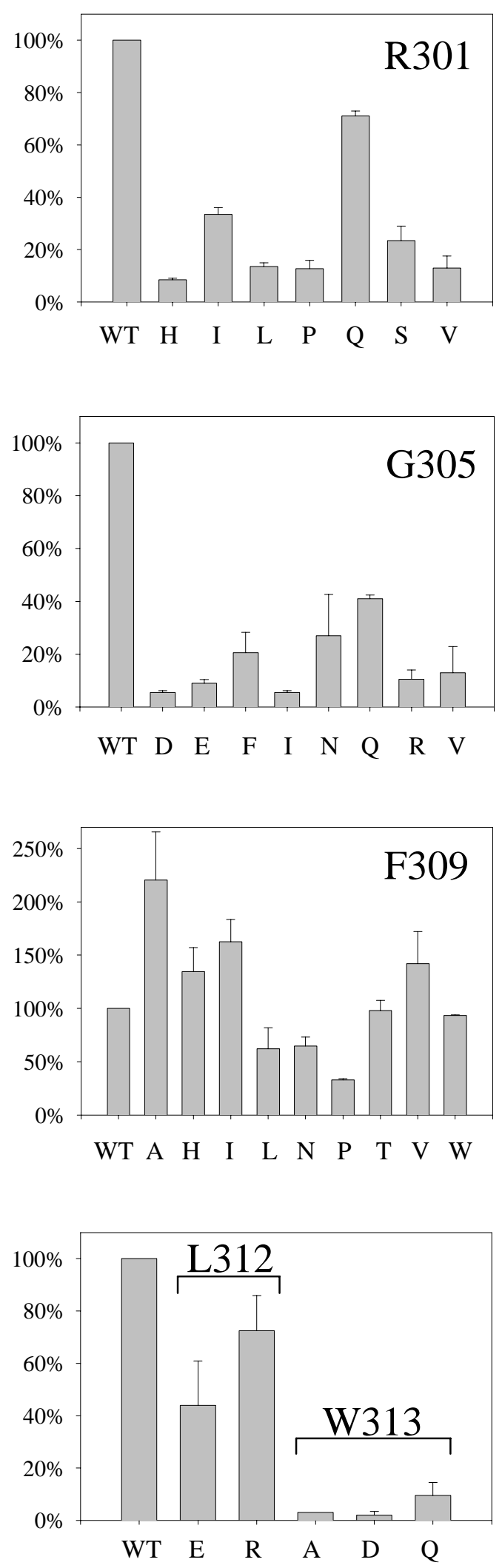
Figure 5

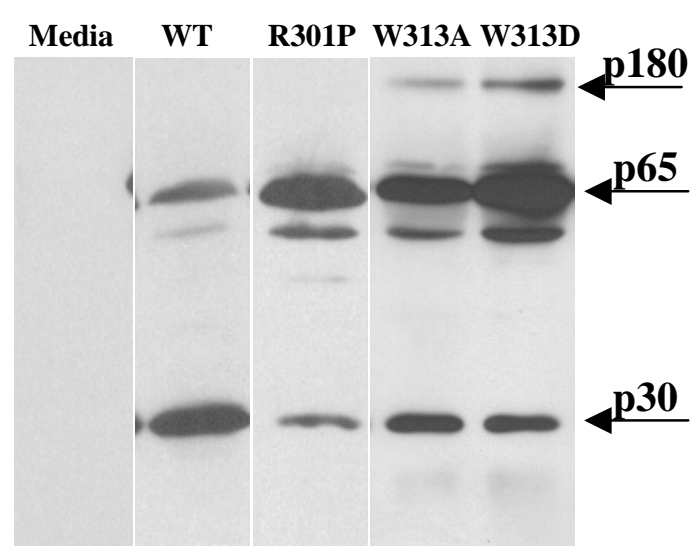




\section{REFERENCES}

1. Bakhanashvili, M., O. Avidan, and A. Hizi. 1996. Mutational studies of human immunodeficiency virus type 1 reverse transcriptase: the involvement of residues 183 and 184 in the fidelity of DNA synthesis. FEBS Lett. 391:257-262.

2. Beard, W. A., K. Bebenek, T. A. Darden, L. Li, R. Prasad, T. A. Kunkel, and S. H. Wilson. 1998. Vertical-scanning mutagenesis of a critical tryptophan in the minor groove binding track of HIV-1 reverse transcriptase. Molecular nature of polymerase-nucleic acid interactions. J. Biol. Chem. 273:30435-30442.

3. Beard, W. A., D. T. Minnick, C. L. Wade, R. Prasad, R. L. Won, A. Kumar, T. A. Kunkel, and S. H. Wilson. 1996. Role of the "helix clamp" in HIV-1 reverse transcriptase catalytic cycling as revealed by alanine-scanning mutagenesis. J. Biol. Chem. 271:12213-12220.

4. Beard, W. A., S. J. Stahl, H. R. Kim, K. Bebenek, A. Kumar, M. P. Strub, S. P. Becerra, T. A. Kunkel, and S. H. Wilson. 1994. Structure/function studies of human immunodeficiency virus type 1 reverse transcriptase. Alanine scanning mutagenesis of an alpha-helix in the thumb subdomain. J. Biol. Chem. 269:28091-28097.

5. Bebenek, K., W. A. Beard, J. R. Casas-Finet, H. R. Kim, T. A. Darden, S. H. Wilson, and T. A. Kunkel. 1995. Reduced frameshift fidelity and processivity of HIV-1 reverse transcriptase mutants containing alanine substitutions in helix $\mathrm{H}$ of the thumb subdomain. J. Biol. Chem. 270:19516-19523. 
6. Bebenek, K., W. A. Beard, T. A. Darden, L. Li, R. Prasad, B. A. Luton, D. G. Gorenstein, S. H. Wilson, and T. A. Kunkel. 1997. A minor groove binding track in reverse transcriptase. Nat. Struct. Biol. 4:194-197.

7. Berman, H. M., J. Westbrook, Z. Feng, G. Gilliland, T. N. Bhat, H. Weissig, I. N. Shindyalov, and P. E. Bourne. 2000. The Protein Data Bank. Nucleic Acids Res. 28:235-242.

8. Chesebro, B., W. Britt, L. Evans, K. Wehrly, J. Nishio, and M. Cloyd. 1983. Characterization of monoclonal antibodies reactive with murine leukemia viruses: use in analysis of strains of friend MCF and Friend ecotropic murine leukemia virus. Virology 127:134-148.

9. Coffin, J. M. 1995. HIV population dynamics in vivo: implications for genetic variation, pathogenesis, and therapy. Science 267:483-489.

10. Ding, J., K. Das, Y. Hsiou, S. G. Sarafianos, A. D. Clark, Jr., A. JacoboMolina, C. Tantillo, S. H. Hughes, and E. Arnold. 1998. Structure and functional implications of the polymerase active site region in a complex of HIV1 RT with a double-stranded DNA template- primer and an antibody Fab fragment at 2.8 A resolution. J. Mol. Biol. 284:1095-1111.

11. Drosopoulos, W. C., and V. R. Prasad. 1996. Increased polymerase fidelity of E89G, a nucleoside analog-resistant variant of human immunodeficiency virus type 1 reverse transcriptase. J. Virol. 70:4834-4838.

12. Gao, G., and S. P. Goff. 1998. Replication defect of moloney murine leukemia virus with a mutant reverse transcriptase that can incorporate ribonucleotides and deoxyribonucleotides. J. Virol. 72:5905-5911. 
13. Gao, H. Q., P. L. Boyer, E. Arnold, and S. H. Hughes. 1998. Effects of mutations in the polymerase domain on the polymerase, RNase $\mathrm{H}$ and strand transfer activities of human immunodeficiency virus type 1 reverse transcriptase. J. Mol. Biol. 277:559-572.

14. Georgiadis, M. M., S. M. Jessen, C. M. Ogata, A. Telesnitsky, S. P. Goff, and W. A. Hendrickson. 1995. Mechanistic implications from the structure of a catalytic fragment of Moloney murine leukemia virus reverse transcriptase. Structure 3:879-892.

15. Ghosh, M., P. S. Jacques, D. W. Rodgers, M. Ottman, J. L. Darlix, and S. F. Le Grice. 1996. Alterations to the primer grip of p66 HIV-1 reverse transcriptase and their consequences for template-primer utilization. Biochemistry 35:85538562.

16. Ghosh, M., J. Williams, M. D. Powell, J. G. Levin, and S. F. Le Grice. 1997. Mutating a conserved motif of the HIV-1 reverse transcriptase palm subdomain alters primer utilization. Biochemistry 36:5758-5768.

17. Gutierrez-Rivas, M., A. Ibanez, M. A. Martinez, E. Domingo, and L. Menendez-Arias. 1999. Mutational analysis of Phe160 within the "palm" subdomain of human immunodeficiency virus type 1 reverse transcriptase. J. Mol. Biol. 290:615-625.

18. Halvas, E. K., E. S. Svarovskaia, and V. K. Pathak. 2000. Development of an In vivo assay to identify structural determinants in murine leukemia virus reverse transcriptase important for fidelity. J. Virol. 74:312-319. 
19. Halvas, E. K., E. S. Svarovskaia, and V. K. Pathak. 2000. The role of murine leukemia virus reverse transcriptase dNTP-binding site in retroviral replication and in vivo fidelity (submitted to J.Virol.).

20. Harris, D., N. Kaushik, P. K. Pandey, P. N. Yadav, and V. N. Pandey. 1998. Functional analysis of amino acid residues constituting the dNTP binding pocket of HIV-1 reverse transcriptase. J. Biol. Chem. 273:33624-33634.

21. Hermann, T., and H. Heumann. 1996. Strained template under the thumbs. How reverse transcriptase of human immunodeficiency virus type 1 moves along its template. Eur. J. Biochem. 242:98-103.

22. Hermann, T., T. Meier, M. Gotte, and H. Heumann. 1994. The 'helix clamp' in HIV-1 reverse transcriptase: a new nucleic acid binding motif common in nucleic acid polymerases. Nucleic Acids Res. 22:4625-4633.

23. Hu, W. S., E. H. Bowman, K. A. Delviks, and V. K. Pathak. 1997. Homologous recombination occurs in a distinct retroviral subpopulation and exhibits high negative interference. J. Virol. 71:6028-6036.

24. Hu, W. S., and H. M. Temin. 1990. Retroviral recombination and reverse transcription. Science 250:1227-1233.

25. Huang, H., R. Chopra, G. L. Verdine, and S. C. Harrison. 1998. Structure of a covalently trapped catalytic complex of HIV-1 reverse transcriptase: implications for drug resistance. Science 282:1669-1675.

26. Jacobo-Molina, A., J. Ding, R. G. Nanni, A. D. Clark, Jr., X. Lu, C. Tantillo, R. L. Williams, G. Kamer, A. L. Ferris, P. Clark, and et al. 1993. Crystal structure of human immunodeficiency virus type 1 reverse transcriptase 
complexed with double-stranded DNA at 3.0 A resolution shows bent DNA. Proc. Natl. Acad. Sci. USA 90:6320-6324.

27. Jacques, P. S., B. M. Wohrl, M. Ottmann, J. L. Darlix, and S. F. Le Grice. 1994. Mutating the "primer grip" of p66 HIV-1 reverse transcriptase implicates tryptophan-229 in template-primer utilization. J. Biol. Chem. 269:26472-26478.

28. Julias, J. G., D. Hash, and V. K. Pathak. 1995. $\mathrm{E}^{-}$vectors: development of novel self-inactivating and self-activating retroviral vectors for safer gene therapy. J. Virol. 69:6839-6846.

29. Julias, J. G., T. Kim, G. Arnold, and V. K. Pathak. 1997. The antiretrovirus drug 3'-azido-3'-deoxythymidine increases the retrovirus mutation rate. J. Virol. 71:4254-4263.

30. Kent, R. B., J. R. Emanuel, Y. Ben Neriah, R. Levenson, and D. E. Housman. 1987. Ouabain resistance conferred by expression of the cDNA for a murine $\mathrm{Na}+$, K+-ATPase alpha subunit. Science 237:901-903.

31. Kim, B., T. R. Hathaway, and L. A. Loeb. 1998. Fidelity of mutant HIV-1 reverse transcriptases: interaction with the single-stranded template influences the accuracy of DNA synthesis. Biochemistry 37:5831-5839.

32. Kim, J. K., C. Palaniappan, W. Wu, P. J. Fay, and R. A. Bambara. 1997. Evidence for a unique mechanism of strand transfer from the transactivation response region of HIV-1. J. Biol. Chem. 272:16769-16777.

33. Kohlstaedt, L. A., J. Wang, J. M. Friedman, P. A. Rice, and T. A. Steitz. 1992. Crystal structure at 3.5 A resolution of HIV-1 reverse transcriptase complexed with an inhibitor. Science 256:1783-1790. 
34. Landau, N. R., K. A. Page, and D. R. Littman. 1991. Pseudotyping with human T-cell leukemia virus type I broadens the human immunodeficiency virus host range. J. Virol. 65:162-169.

35. Latham, G. J., E. Forgacs, W. A. Beard, R. Prasad, K. Bebenek, T. A. Kunkel, S. H. Wilson, and R. S. Lloyd. 2000. Vertical-scanning mutagenesis of a critical tryptophan in the "minor groove binding track" of HIV-1 reverse transcriptase. Major groove DNA adducts identify specific protein interactions in the minor groove. J. Biol. Chem. 275:15025-15033.

36. Mansky, L. M., and H. M. Temin. 1995. Lower in vivo mutation rate of human immunodeficiency virus type 1 than that predicted from the fidelity of purified reverse transcriptase. J. Virol. 69:5087-5094.

37. Mansky, L. M., and H. M. Temin. 1994. Lower mutation rate of bovine leukemia virus relative to that of spleen necrosis virus. J. Virol. 68:494-499.

38. Martin-Hernandez, A. M., E. Domingo, and L. Menendez-Arias. 1996. Human immunodeficiency virus type 1 reverse transcriptase: role of Tyr115 in deoxynucleotide binding and misinsertion fidelity of DNA synthesis. EMBO J. 15:4434-4442.

39. Martin-Hernandez, A. M., M. Gutierrez-Rivas, E. Domingo, and L. Menendez-Arias. 1997. Mispair extension fidelity of human immunodeficiency virus type 1 reverse transcriptases with amino acid substitutions affecting Tyr115. Nucleic Acids Res. 25:1383-1389. 
40. Miller, A. D., J. V. Garcia, N. von Suhr, C. M. Lynch, C. Wilson, and M. V. Eiden. 1991. Construction and properties of retrovirus packaging cells based on gibbon ape leukemia virus. J. Virol. 65:2220-2224.

41. Najmudin, S., M. L. Cote, D. Sun, S. Yohannan, S. P. Montano, J. Gu, and M. M. Georgiadis. 2000. Crystal structures of an N-terminal fragment from Moloney murine leukemia virus reverse transcriptase complexed with nucleic acid: functional implications for template-primer binding to the fingers domain. J. Mol. Biol. 296:613-632.

42. Palaniappan, C., M. Wisniewski, P. S. Jacques, S. F. Le Grice, P. J. Fay, and R. A. Bambara. 1997. Mutations within the primer grip region of HIV-1 reverse transcriptase result in loss of RNase H function. J. Biol. Chem. 272:11157-11164.

43. Parthasarathi, S., A. Varela-Echavarria, Y. Ron, B. D. Preston, and J. P. Dougherty. 1995. Genetic rearrangements occurring during a single cycle of murine leukemia virus vector replication: characterization and implications. J. Virol. 69:7991-8000.

44. Pathak, V. K., and H. M. Temin. 1990. Broad spectrum of in vivo forward mutations, hypermutations, and mutational hotspots in a retroviral shuttle vector after a single replication cycle: deletions and deletions with insertions. Proc. Natl. Acad. Sci. USA 87:6024-6028.

45. Pathak, V. K., and H. M. Temin. 1990. Broad spectrum of in vivo forward mutations, hypermutations, and mutational hotspots in a retroviral shuttle vector after a single replication cycle: substitutions, frameshifts, and hypermutations. Proc. Natl. Acad. Sci. USA 87:6019-6023. 
46. Powell, M. D., W. A. Beard, K. Bebenek, K. J. Howard, S. F. Le Grice, T. A. Darden, T. A. Kunkel, S. H. Wilson, and J. G. Levin. 1999. Residues in the alphaH and alphaI helices of the HIV-1 reverse transcriptase thumb subdomain required for the specificity of RNase $\mathrm{H}$ - catalyzed removal of the polypurine tract primer. J. Biol. Chem. 274:19885-19893.

47. Powell, M. D., M. Ghosh, P. S. Jacques, K. J. Howard, S. F. Le Grice, and J. G. Levin. 1997. Alanine-scanning mutations in the "primer grip" of p66 HIV-1 reverse transcriptase result in selective loss of RNA priming activity. J. Biol. Chem. 272:13262-13269.

48. Preston, B. D., B. J. Poiesz, and L. A. Loeb. 1988. Fidelity of HIV-1 reverse transcriptase. Science 242:1168-1171.

49. Roberts, J. D., B. D. Preston, L. A. Johnston, A. Soni, L. A. Loeb, and T. A. Kunkel. 1989. Fidelity of two retroviral reverse transcriptases during DNAdependent DNA synthesis in vitro. Mol. Cell. Biol. 9:469-476.

50. Sambrook, J., E. F. Fritsch, and T. Maniatis. 1989. Molecular cloning: a laboratory manual, 2nd ed. Cold Spring Harbor Laboratory Press, Cold Spring Harbor, N.Y.

51. Sarafianos, S. G., V. N. Pandey, N. Kaushik, and M. J. Modak. 1995. Glutamine 151 participates in the substrate dNTP binding function of HIV-1 reverse transcriptase. Biochemistry 34:7207-7216.

52. Schinazi, R. F., B. A. Larder, and J. W. Mellors. 2000. Mutations in retroviral genes assosiated with drug resistance: 2000-2001 update. Internat. Antiviral News 8:65-91. 
53. Smerdon, S. J., J. Jager, J. Wang, L. A. Kohlstaedt, A. J. Chirino, J. M. Friedman, P. A. Rice, and T. A. Steitz. 1994. Structure of the binding site for nonnucleoside inhibitors of the reverse transcriptase of human immunodeficiency virus type 1. Proc. Natl. Acad. Sci. USA 91:3911-3915.

54. Svarovskaia, E. S., K. A. Delviks, C. K. Hwang, and V. K. Pathak. 2000. Structural determinants of murine leukemia virus reverse transcriptase that affect the frquency of template switching. J. Virol. 74:7171-7178.

55. Telesnitsky, A., and S. P. Goff. 1993. RNase H domain mutations affect the interaction between Moloney murine leukemia virus reverse transcriptase and its primer-template. Proc. Natl. Acad. Sci. USA 90:1276-1280.

56. Temin, H. M. 1993. Retrovirus variation and reverse transcription: abnormal strand transfers result in retrovirus genetic variation. Proc. Natl. Acad. Sci. USA 90:6900-6903.

57. Wainberg, M. A., W. C. Drosopoulos, H. Salomon, M. Hsu, G. Borkow, M. Parniak, Z. Gu, Q. Song, J. Manne, S. Islam, G. Castriota, and V. R. Prasad. 1996. Enhanced fidelity of 3TC-selected mutant HIV-1 reverse transcriptase. Science 271:1282-1285.

58. Wisniewski, M., C. Palaniappan, Z. Fu, S. F. Le Grice, P. Fay, and R. A. Bambara. 1999. Mutations in the primer grip region of HIV reverse transcriptase can increase replication fidelity. J. Biol. Chem. 274:28175-28184.

59. Wohrl, B. M., R. Krebs, S. H. Thrall, S. F. J. Le Grice, A. J. Scheidig, and R. S. Goody. 1997. Kinetic analysis of four HIV-1 reverse transcriptase enzymes 
mutated in the primer grip region of p66. Implications for DNA synthesis and dimerization. J. Biol. Chem. 272:17581-17587. 


\section{CHAPTER 4}

\section{WILD-TYPE AND YMDD MUTANT OF MURINE LEUKEMIA VIRUS REVERSE TRANSCRIPTASE ARE RESISTANT TO $2^{\prime}, 3^{\prime}$-DIDEOXY-3'-THIACYTIDINE}

ELIAS K. HALVAS, ${ }^{1}$ EVGUENIA S. SVAROVSKAIA, ${ }^{1,2}$ ERIC O. FREED, ${ }^{3}$ AND VINAY K. PATHAK ${ }^{1,2}$

Mary Babb Randolph Cancer Center and Department of Biochemistry, West Virginia University, Morgantown, West Virginia 26506, ${ }^{1}$ HIV Drug Resistance Program, National Cancer Institute, FCRDC, Frederick, Maryland 21702-1201, ${ }^{2}$ and Laboratory of Molecular Microbiology, National Institute of Allergy and Infectious Diseases, National Institutes of Health, Bethesda, Maryland 20892-0460

Experiments, described in this chapter, are published as a manuscript in Journal of Virology, July. 2000, 74(14): 6669-74. 


\begin{abstract}
The antiretroviral nucleoside analog $2^{\prime}, 3^{\prime}$-dideoxy-3'-thiacytidine (3TC) is a potent inhibitor of wild-type human immunodeficiency virus type 1 (HIV-1) reverse transcriptase (RT). A methionine-to-valine or methionine-to-isoleucine substitution at residue 184 in the HIV-1 YMDD motif, which is located at the RT active site, leads to a high level of resistance to 3TC. We sought to determine whether 3TC can inhibit the replication of wild-type murine leukemia virus (MLV) RT, which contains V223 at the YVDD active site motif, and of the V223M, V223I, V223A, and V223S mutant RTs. Surprisingly, the wild-type and all four of the V223 mutants of MLV RT were highly resistant to 3TC. These results indicate that determinants outside the YVDD motif of MLV RT confer a high level of resistance to 3TC. Therefore, structural differences among similar RTs might result in widely divergent sensitivities to antiretroviral nucleoside analogs.

\section{INTRODUCTION}

Currently, 14 antiviral drugs have been approved for clinical use to combat human immunodeficiency virus type 1 (HIV-1) infections $(11,54)$. When used in combination, many of these drugs have been shown to prolong the life expectancy of infected individuals and slow the progression of $\operatorname{AIDS}(12,14,20,21,40,42)$. Most of these drugs, both nucleoside and nonnucleoside inhibitors, have been designed to target reverse transcriptase $(\mathrm{RT})(2,10,53)$. One of the compounds used in combination therapy is the nucleoside analog $2^{\prime}, 3^{\prime}$-dideoxy-3'-thiacytidine $(3 \mathrm{TC})(4,20,21,37,51)$. The triphosphate form of 3TC inhibits reverse transcription through chain termination of DNA polymerization, a function carried out by RT, and therefore has been observed to be 
a potent inhibitor of both HIV type 1 and type 2 replication $(2,9,10)$. In addition to the clinical benefits $(20,21,37,46)$ associated with the use of this nucleoside analog, 3TC exhibits low toxicity, even at millimolar concentrations $(7,9,15,36)$.

All of the drugs being used for therapy to combat HIV-1 infections eventually result in drug-resistant mutants, thereby allowing for the progression of the disease. Resistance to $3 \mathrm{TC}$ is no exception and is characterized by a mutation at the methionine 184 position of the Tyr-Met-Asp-Asp (YMDD) motif found in HIV-1 RT (5, 17, 31, 48, 55). The YXDD motif, where $\mathrm{X}$ is a variable amino acid, is highly conserved among the many viral RNA polymerases as well as RNA-dependent DNA polymerases (43). For example, Rous sarcoma virus RT contains the YMDD motif, the RTs of retroelements such as 297 (Gypsy-like group) and Int 32 (Line-like group) contain both the YLDD and YADD sequences, and the poliovirus RNA polymerase contains the YGDD motif (43). Mutations in the YXDD motif can abolish enzymatic activity and alter the processivity and fidelity of RT $(3,6,23,27,39)$. The prevalent mutation in HIV-1 RT associated with 3TC resistance is the M184V substitution $(17,48,55)$, which confers a level of resistance 1000 times greater than that displayed by the wild-type enzyme $(17,55)$. It has been observed that the M184I variant, which is resistant to 3TC but is less catalytically active, is selected first after initiation of $3 \mathrm{TC}$ treatment $(5,6,31,55)$, and then is replaced by the M184V variant after long-term treatment with 3TC.

Mutations in the motif analogous to the HIV-1 RT YMDD domain are also correlated with resistance to $3 \mathrm{TC}$ in other retroviruses. The YVDD, YIDD, and YTDD motifs are selected during 3TC treatment of cells infected with simian immunodeficiency virus (SIV) or feline immunodeficiency virus (FIV) $(8,49)$. In addition, the YVDD and 
YIDD motifs arise during 3TC treatment of hepatitis B virus (HBV)-infected cells and patients (1). The selection for mutations in the YXDD motif in other retroviruses as well as in HBV has suggested that this determinant is widely associated with 3TC sensitivity.

Other mutations in HIV-1 and FIV RTs have been implicated to confer dual resistance to 3TC and other nucleoside analogs. Specifically, the E89G and G333E mutations in HIV-1 RT are correlated with dual resistance in tissue culture to 3TC and either phosphonoformic acid or 3'-azido-3'-deoxythymidine (AZT), respectively $(29,45)$. The K65R mutation in HIV-1 RT is associated with resistance to 3TC and 2',3'dideoxycytidine (ddC) (19). Finally, the P156S mutation in FIV RT appears to confer resistance to both AZT and 3TC (50).

Although HIV-1 and MLV RTs share only 25\% amino acid sequence identity, the two proteins are structurally similar (18). Comparison of the finger and palm domains in HIV-1 and MLV RT crystal structures reveals similar tertiary structures (18, 25,34 ). In addition, many of the sequence motifs present in HIV-1 RT, such as the YXDD motif, the dNTP-binding site, and the conserved Leu-Pro-Gln-Gly (LPQG) motif, are also present in MLV RT. Importantly, the antiretroviral nucleoside analogs AZT, ddC, 2',3'-dideoxyinosine (ddI), and 2', $3^{\prime}$-didehydro-3'-deoxythimidine (d4T), which inhibit HIV-1 RT, also inhibit MLV RT (52). Therefore, since wild-type MLV RT contains the YVDD motif, it was expected and recently shown to be resistant to 3TC (44).

Based on the similarities between the HIV-1 and MLV RTs, we hypothesized that the YMDD mutant of MLV RT would be sensitive to 3TC and the YIDD mutant would be resistant. To test this hypothesis, we generated viruses containing wild-type MLV RT 
as well as several mutants at position V223 within the YVDD motif and compared the titers of these viruses in several different target cells in the presence or absence of 3TC.

\section{RESULTS AND DISCISSION}

MLV mutants, target cells, and the ANGIE P cell line. The construction of the V223A, V223I, V223M, and V223S mutants was described previously (23). The mutants were generated from the parent plasmid pLGPS (Fig. 1A), which expresses the MLV gag and pol genes from a truncated MLV long terminal repeat ( $\triangle \mathrm{LTR})$ promoter (41).

The targets of infection in this study included the murine fibroblast cell line NIH 3T3 and the human osteosarcoma cell line 143B (both obtained from the American Type Culture Collection). The ANGIE P cell line (Fig. 1B) is a D17-based (dog osteosarcoma) cell line expressing the construct pSV-A-MLVenv (obtained from the NIH AIDS Research and Reference Program) and pGA-1, an MLV-based retroviral vector (Fig. 1A) $(23,38)$. The expression construct pSV-A-MLVenv encodes the amphotropic MLV envelope gene, whereas pGA-1 expresses the bacterial $\beta$-galactosidase gene (lacZ) from the LTR promoter. In addition, pGA-1 also contains the neomycin phosphotransferase gene (neo), which is utilized as a selectable marker during infection. All cells were maintained in Dulbecco's modified Eagle's medium (ICN Biochemicals) supplemented with penicillin (50 U/ml; Gibco), streptomycin ( $50 \mu \mathrm{g} / \mathrm{ml}$; Gibco), and bovine calf serum (6\% for ANGIE P and 143B cells or 10\% for NIH 3T3 cells; HyClone Laboratories).

Protocol for determining sensitivity of MLV RT to 3TC. The approach used to determine the sensitivity of MLV RT to 3TC is outlined in Fig. 1B. Briefly, either wildtype or mutated pLGPS along with $\mathrm{pSV} \alpha 3.6$, a plasmid that confers resistance to ouabain (30), were cotransfected into the ANGIE P cell line. Transfections were carried out by 
the previously described dimethyl sulfoxide-Polybrene method (28) and the transfected cells were then selected for resistance to $10^{-7} \mathrm{M}$ ouabain.

To determine the sensitivity of the MLV RTs to 3TC, we separately pooled and expanded more than 500 ouabain-resistant colonies for the wild-type and V223 mutants. For each pLGPS construct, $5 \times 10^{6}$ ouabain-resistant cells were plated on $100-\mathrm{mm}$ diameter dishes and the medium was changed $24 \mathrm{~h}$ later. Virus was harvested after another $24 \mathrm{~h}$ and serially diluted. In the presence of Polybrene $(50 \mu \mathrm{g} / \mathrm{ml})$, the virus was used to infect either NIH 3 T3 or 143B cells for $4 \mathrm{~h}$. The target cells were plated at a density of $1-2 \times 10^{5}$ cells per 60 -mm-diameter dish. In experiments conducted in the presence of the drug, the target cells were incubated with $10 \mu \mathrm{M} 3 \mathrm{TC} 4 \mathrm{~h}$ prior to infection, $4 \mathrm{~h}$ during infection, and $24 \mathrm{~h}$ post infection. The $3 \mathrm{TC}$ concentration used in this study was 15 - to 4000 -fold higher than the mean $50 \%$ inhibitory concentration [ $\mathrm{IC}_{50}$ ], ranging between $2.5 \mathrm{nM}$ to $0.67 \mu \mathrm{M}$, which was previously shown to inhibit several different strains of HIV-1 (9). The infected cells were then subjected to selection with G418, an analog of neomycin $(600 \mu \mathrm{g} / \mathrm{ml}$ for $143 \mathrm{~B}$ and D17 cells and $1.2 \mathrm{mg} / \mathrm{ml}$ for NIH 3T3 cells) $24 \mathrm{~h}$ after infection. The effect of 3TC treatment on MLV replication (wildtype and V223 mutants) was determined by the number of drug-resistant colonies obtained in the presence or absence of 3TC.

Comparison of viral titers in the presence or absence of 3TC. Viral titers were determined by quantitation of G418-resistant NIH 3T3 and 143B cells after infection (the data are summarized in Table 1). ANGIE P cells transfected with wild-type pLGPS or the V223A, V223I, V223M, or V223S mutant were previously shown to produce infectious viral particles, and target cells infected with these viruses were expected to 
confer resistance to G418 (23). Two to four independent infections of NIH 3T3 and 143B cells, in the absence or presence of 3TC, were performed with virus containing either the wild-type pLGPS or one of the four V223 mutants.

Infection of NIH 3T3 cells with the wild-type virus harvested from a single pool produced titers that ranged from $2.7 \times 10^{4}$ to $1.3 \times 10^{5} \mathrm{CFU} / \mathrm{ml}$ in the absence of 3TC. Treatment of the target cells with 3TC had no significant effect on the titers of the wildtype virus (82\%, relative to the untreated control). Similarly, infection of NIH 3 T3 cells with the V223M mutant produced titers that ranged from $1.6 \times 10^{2}$ to $9.2 \times 10^{4} \mathrm{CFU} / \mathrm{ml}$ in the absence of 3TC after harvesting virus from two different virus-producing pools. Treatment of the target cells with 3TC did not substantially affect the titers of the V223M mutant virus ( $57 \%$, relative to the untreated control) when compared to the inhibition of the luciferase-expressing HIV-1-based vector pNLuc (Fig. 2C). The 2-fold change observed in the titers in the absence or presence of 3TC is probably not biologically relevant due to the inherent variation that occurs during infections (23). We also assessed the sensitivity to 3TC of other V223 mutants (V223A, V223I, and V223S) in NIH 3T3 cells; the results were similar to those obtained with the wild-type and V223M mutant viruses. In summary, viral titers in the presence or absence of 3TC varied by only 2-fold, suggesting that 3TC did not substantially inhibit either the wild-type or V223 mutant RTs during infection of NIH $3 \mathrm{~T} 3$ cells.

To determine whether the lack of inhibition to MLV replication with 3TC treatment was specific to NIH 3 T3 cells, we also tested infection of 143B cells and D17 cells. Infection of 143B cells with virus containing either the wild-type or V223M mutant RT produced results similar to those obtained with NIH 3T3 cells. Virus obtained 
from wild-type pLGPS produced viral titers that ranged from $3.4 \times 10^{2}$ to $1.9 \times 10^{3}$

$\mathrm{CFU} / \mathrm{ml}$ in the absence of 3TC. Treatment of the target cells with 3TC had no significant effect on the titers of the wild-type virus (110\%, relative to the untreated control). In addition, infection of 143B cells with virus containing the V223M mutant RT produced viral titers ranging from $1.0 \times 10^{1}$ to $2.5 \times 10^{2} \mathrm{CFU} / \mathrm{ml}$ in the absence of $3 \mathrm{TC}$ and $0.5 \times$ $10^{1}$ to $1.3 \times 10^{2} \mathrm{CFU} / \mathrm{ml}$ in the presence of $3 \mathrm{TC}(57 \%$, relative to the untreated control). Similar results were obtained with D17 cells (data not shown).

Activation of 3TC in target cells. The results obtained with viruses generated and harvested from the ANGIE P cells that had been transfected with either the wild-type or V223 mutant constructs showed that all MLV RTs were resistant to 3TC (Table 1). There are two possible explanations for these results. First, structural differences between MLV RT and HIV-1 RT might account for the resistance of MLV RT to 3TC. Second, the uptake and/or phosphorylation of the drug by the target cells might be inefficient. To address these possibilities, we generated infectious HIV-1 particles and used them to infect the various target cells as previously described $(32,33)$ (Fig. 2A and 2B). Briefly, 293T (human embryonic kidney) cells were cotransfected with pNLuc and pSV-A-MLVenv. Pseudotyped virus stocks were harvested and used to infect NIH 3T3, HeLa, 143B, and D17 cells in the presence or absence of $10 \mu \mathrm{M}$ 3TC. The target cells infected in the presence of drug were incubated with 3TC for $4 \mathrm{~h}$ prior to infection, $4 \mathrm{~h}$ during infection, and $24 \mathrm{~h}$ post infection. Two days post infection, the cells were lysed and the amount of luciferase activity present in the lysates was measured with a luminometer (Tropix) (Fig. 2B). 
Infection of NIH 3T3, 143B, and HeLa cells with the pNLuc-derived virus was decreased 20-fold by 3TC treatment (Fig. 2C). The D17 cells exhibited a 4-fold decrease in pNLuc expression relative to no $3 \mathrm{TC}$ treatment. Thus, in the same cell lines in which MLV infectivity was not substantially affected, HIV-1 infectivity was significantly reduced. These results indicate that the lack of an effect of 3TC on MLV infectivity was not due to problems associated with the uptake, phosphorylation, or other mechanisms that interfered with the inhibitory activity of the nucleoside analog in these target cells. The less efficient inhibition of pNLuc expression in the D17 cells in comparison to the other target cells (Fig. 2C) could have been caused by either reduced uptake or phosphorylation of 3TC in D17 cells. Alternatively, this cell line might actively export the nucleoside analog, thus reducing its efficacy. Regardless of the mechanism, HIV-1 infection of different cell types in vivo might display divergent susceptibilities to 3TC or other RT inhibitors. Thus, the data obtained with the D17 cell line might have implications for drug therapy in HIV-1-infected patients.

Mechanism of 3TC resistance in MLV RT. 3TC resistance arises in both retroviral (HIV-1, SIV, and FIV) and nonretroviral (HBV) polymerases with catalytic sites containing the YMDD motif $(1,8,17,31,48,49,55)$. This resistance usually results from a substitution of methionine to threonine, isoleucine or valine. Based on these observations, we expected that the wild-type MLV RT containing the YVDD motif would be resistant (44) and the V223M mutant would be sensitive to 3TC. It was therefore surprising that both the wild-type and the V223M mutant were highly resistant to $3 \mathrm{TC}$. 
The mechanism by which the M184V mutant of HIV-1 RT confers resistance to 3TC is unclear. The methionine-to-isoleucine substitution at position 184 in HIV-1 RT results in a repositioning of the template-primer complex, and this rearrangement might result in a misalignment of the $3 \mathrm{TC}$ triphosphate with the template, resulting in a decrease in the turnover rate (47). Molecular modeling of the wild-type and M184I mutant of HIV-1 RT has also suggested that steric hindrance between the $\beta$-Loxathiolane ring of 3TC triphosphate and the $\beta$-branched amino acids (valine, isoleucine, and threonine) at position 184 interferes with $3 \mathrm{TC}$ binding $(25,47)$. It is important to note that the proposed steric hinderance model does not preclude $3 \mathrm{TC}$ binding to RT in a mode that is unfavorable to its incorporation. In this regard, Feng and Anderson (13) reported that $3 \mathrm{TC}$ triphosphate binds to the M184V mutant with a much higher Kd value $(5.2 \mu \mathrm{M})$ relative to the wild type HIV-1 RT $(0.24 \mu \mathrm{M})$. Similarly, Wilson et al (56) suggested that $\beta$-L-2', $3^{\prime}$-dideoxy-5-fluoro-3'-thiacytidine, a nucleoside analog that is structurally similar to 3TC, binds with a higher affinity to the wild type HIV-1 RT than the M184V mutant. However, Krebs and coworkers did not find a substantial difference in the $\mathrm{Kd}$ values for 3TC binding to wild type and M184V mutants (35). Recent evidence indicates that the 3TC can bind to the M184V and M184I mutants of HIV-1 RT, which results in a conformational change in the enzyme that affects the nature of RNase H cleavages (16).

The fact that both the YMDD and YVDD motifs are highly resistant to 3TC strongly suggests that other structural determinants of MLV RT may interfere with the nature of 3TC binding through steric hindrance. The previous observation that MLV RT is sensitive to $\mathrm{ddC}(52)$ suggests that the steric hindrance involves the $\beta$-L-oxathiolane 
ring of 3TC. In accordance with the relatively low ( 25\%) amino acid sequence identity between the MLV and HIV-1 RTs (18), structural alterations at or near the active site not related to the YVDD motif of MLV RT may lead to steric hindrance and prevent 3TC binding. A comparison of distances between residues of the YXDD motif and dNTP binding pocket of HIV-1 and MLV RTs reveals substantial differences (18, 24). Distances in MLV RT, specifically between the residues of the YVDD motif and K103, which is equivalent to K65 of HIV-1 RT (22), are more than 2 angstroms longer when compared to HIV-1 RT. It should be noted that the K65 residue of HIV-1 RT is associated with dual resistance to $3 \mathrm{TC}$ and ddC (19). These and other structural differences might contribute to the divergent sensitivities to 3TC.

Additionally, amino acid differences between the MLV and HIV-1 RTs around the dNTP-binding pocket may also provide the steric hindrance needed to confer 3TC resistance. Specifically, the MLV RT possesses a phenylalanine at position 155 , which is equivalent to the tyrosine 115 in HIV-1 RT. The Y115 of HIV-1 RT has been shown to interact with the ribose ring of a dNTP substrate (25). Therefore, substitutions of the phenylalanine for the tyrosine in MLV RT dNTP binding site may alter the nature of 3TC binding.

It is also conceivable that the structural differences near the active site or dNTP binding site between MLV and HIV-1 RTs, alter the affinity or nature of binding of 3TC triphosphate relative to $\mathrm{dCTP}$ by a mechanism not involving steric hinderance. The nature of the structural differences that confer high-level resistance to 3TC despite the presence of a YMDD motif appears to be unique to MLV RT, since several other retroviral RTs as well as HBV polymerase display sensitivity to 3TC when the YMDD 
motif is present $(1,8,49)$. Understanding the nature of the structural differences that lead to 3TC resistance in MLV RT might provide insights into the general mechanisms by which retroviral RTs acquire resistance to nucleotide analogs.

\section{ANKNOLEGMENTS}

We especially thank Wei-Shau Hu for critical reading of the manuscript and valuable intellectual input and discussions throughout the project. We also especially thank Stephen H. Hughes for communicating unpublished results, intellectual input, and critical reading of the manuscript. We also thank Benjamin Beasley, Sara Cheslock, Que Dang, Krista Delviks, Carey Hwang, Timur Kabdulov, Terence Rhodes, Yegor Voronin, and Wen Hui Zhang for the critical reading of this manuscript and discussion of results. Finally, we extend our thanks to Ann Arthur for her editorial expertise and revisions.

This work was supported in part by Public Health Service grant CA58875 from the National Institutes of Health, HIV Drug Resistance Program, National Cancer Institute, and Laboratory of Molecular Microbiology, National Institute of Allergy and Infectious Diseases, National Institutes of Health. 
TABLE 1. The effect of 3TC treatment on MLV replication

\begin{tabular}{|c|c|c|c|c|c|}
\hline $\begin{array}{l}\text { Target } \\
\text { cells }\end{array}$ & $\begin{array}{l}\text { MLV } \\
\text { RT }^{a}\end{array}$ & $\begin{array}{c}\text { Expt. } \\
\text { no. }\end{array}$ & $\begin{array}{l}-3 \mathrm{TC} \text { titer } \\
(\mathrm{CFU} / \mathrm{ml})^{b}\end{array}$ & $\begin{array}{l}\text { + 3TC titer } \\
(\mathrm{CFU} / \mathrm{ml})^{c}\end{array}$ & 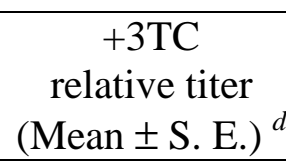 \\
\hline \multirow[t]{19}{*}{ NIH 3T3 } & WT & 1 & $3.7 \times 10^{4}$ & $2.6 \times 10^{4}$ & 0.70 \\
\hline & & 2 & $2.7 \times 10^{4}$ & $1.5 \times 10^{4}$ & 0.56 \\
\hline & & 3 & $7.2 \times 10^{4}$ & $9.2 \times 10^{4}$ & 1.28 \\
\hline & & 4 & $1.3 \times 10^{5}$ & $9.5 \times 10^{4}$ & 0.73 \\
\hline & & Avg & & & $0.82 \pm 0.16$ \\
\hline & V223M & 1 & $2.9 \times 10^{2}$ & $1.4 \times 10^{2}$ & 0.48 \\
\hline & & 2 & $1.6 \times 10^{2}$ & $1.0 \times 10^{2}$ & 0.63 \\
\hline & & 3 & $9.2 \times 10^{4}$ & $6.9 \times 10^{4}$ & 0.75 \\
\hline & & 4 & $2.4 \times 10^{4}$ & $1.0 \times 10^{4}$ & 0.42 \\
\hline & & Avg & & & $0.57 \pm 0.07$ \\
\hline & V223I & 1 & $3.4 \times 10^{4}$ & $2.5 \times 10^{4}$ & 0.74 \\
\hline & & 2 & $2.6 \times 10^{4}$ & $3.3 \times 10^{4}$ & 1.27 \\
\hline & & Avg & & & $1.01 \pm 0.27$ \\
\hline & V223A & 1 & $1.3 \times 10^{2}$ & $1.9 \times 10^{2}$ & 1.46 \\
\hline & & 2 & $2.1 \times 10^{2}$ & $2.0 \times 10^{2}$ & 0.95 \\
\hline & & Avg & & & $1.21 \pm 0.26$ \\
\hline & V223S & 1 & $4.3 \times 10^{4}$ & $4.3 \times 10^{4}$ & 1.00 \\
\hline & & 2 & $8.3 \times 10^{3}$ & $1.2 \times 10^{4}$ & 1.45 \\
\hline & & Avg & & & $1.23 \pm 0.23$ \\
\hline \multirow[t]{10}{*}{ 143B } & WT & 1 & $1.9 \times 10^{3}$ & $1.4 \times 10^{3}$ & 0.74 \\
\hline & & 2 & $3.4 \times 10^{2}$ & $5.8 \times 10^{2}$ & 1.70 \\
\hline & & 3 & $4.3 \times 10^{2}$ & $3.5 \times 10^{2}$ & 0.8 \\
\hline & & 4 & $1.2 \times 10^{3}$ & $1.3 \times 10^{3}$ & 1.10 \\
\hline & & Avg & & & $1.10 \pm 0.23$ \\
\hline & V223M & 1 & $2.5 \times 10^{2}$ & $1.3 \times 10^{2}$ & 0.52 \\
\hline & & 2 & $2.0 \times 10^{2}$ & $8.3 \times 10^{1}$ & 0.42 \\
\hline & & 3 & $1.3 \times 10^{1}$ & $1.1 \times 10^{1}$ & 0.85 \\
\hline & & 4 & $1.0 \times 10^{1}$ & $0.5 \times 10^{1}$ & 0.50 \\
\hline & & Avg & & & $0.57 \pm 0.09$ \\
\hline
\end{tabular}

${ }^{a}$ The genotype of MLV virus generated from transfection of pLGPS (wild-type or V223 mutants of MLV RT) into the ANGIE P encapsidating cell line.

${ }^{b}$ The titer obtained from infections in the absence of 3TC. The virus titer for each experimental group was determined by serial dilutions. In experiments using NIH 
$3 \mathrm{~T} 3$ target cells, four independent infections were performed with virus containing the wild-type or V223M mutant RT, whereas two independent infections were performed for the V223A, V223I, and V223S RTs. In experiments using 143B target cells, four independent infections were performed with virus containing the wild-type or V223M mutant RT.

${ }^{c}$ The titer obtained from infections in the presence of 3TC. The virus titer for each experimental group was determined by serial dilutions. Four independent infections were performed in the presence of $3 \mathrm{TC}(10 \mu \mathrm{M})$ as described in footnote $b$ for virus containing either the wild-type or V223M RT. The 3TC treatment included incubation of the target cells $4 \mathrm{~h}$ prior to infection, $4 \mathrm{~h}$ during infection, and $24 \mathrm{~h}$ post infection.

${ }^{d}$ The $+3 \mathrm{TC}$ relative titer was calculated as follows: [titer obtained in the presence of $3 \mathrm{TC}] \div[$ titer obtained in the absence of $3 \mathrm{TC}]$. S. E. represents the standard error. 


\section{FIGURE LEGENDS AND FIGURES}

\section{FIG. 1. Structures of MLV-based constructs and protocol used to determine}

sensitivity to 3TC. (A) Structures of the MLV-based vector pGA-1 and the gag-pol expression construct pLGPS. The pGA-1 vector contains both LTRs and all cis-acting elements of MLV and transcribes the E. coli lacZ and neo from the LTR promoter. The internal ribosomal entry site (IRES) (26) of encephalomyocarditis virus is used to express neo. The construct pLGPS expresses the MLV gag-pol gene from a truncated viral LTR. (B) Experimental protocol. ANGIE P, a D17-based cell line expressing pGA-1 and a vector carrying the amphotropic MLV env gene (pSV-A-MLVenv), was separately transfected with either the wild-type (V223) or mutated (V223A, V223I, V223M, and V223S) pLGPS constructs. Virus from pools of these transfected ANGIE P cells was harvested and used to infect target cells (NIH $3 \mathrm{~T} 3$ or 143B cells) for $4 \mathrm{~h}$ in the presence or absence of 3TC $(10 \mu \mathrm{M})$. The target cells were also treated with $3 \mathrm{TC}(10 \mu \mathrm{M}) 4 \mathrm{~h}$ prior to infection and $24 \mathrm{~h}$ post infection in the drug treatment groups. (C) Measurement of virus titer. The percent change in the virus titers of the wild-type and V223M mutant was measured in the absence or presence of $3 \mathrm{TC}(10 \mu \mathrm{M})$. Two to four independent experiments were performed for NIH $3 \mathrm{~T} 3$ and 143B cells. Virus titers observed in the absence of 3TC treatment were defined as 100\%. Error bars represent the standard error.

FIG. 2. Structure of HIV-1-based constructs and activation of 3TC in NIH 3T3, HeLA, 143B, and D17 cell lines. (A) Structure of the HIV-1-based luciferaseexpression vector pNLuc, which contains both LTRs, other cis-acting elements required for viral replication, and the gag-pol gene of HIV-1. pNLuc expresses the luciferase reporter gene (luc) from the LTR promoter. (B) Experimental protocol. The vectors 
pNLuc and pSV-A-MLVenv were cotransfected into 293T cells and pseudotyped virus was used to infect various target cells (NIH 3T3, HeLA, 143B, and D17) for $4 \mathrm{~h}$ in the presence or absence of 3TC $(10 \mu \mathrm{M})$. The target cells were also treated with 3TC (10 $\mu \mathrm{M}) 4 \mathrm{~h}$ prior to infection and $24 \mathrm{~h}$ post infection in the drug treatment groups. (C) Measurement of chemiluminescence. The percent of chemiluminescence was measured in the absence or presence of $3 \mathrm{TC}(10 \mu \mathrm{M})$. At least two independent experiments were performed for NIH 3T3, HeLA, 143B, and D17 cells. Chemiluminescence measurements in the absence of 3TC treatment were defined as $100 \%$. Error bars represent the standard error. The standard error bars for both NIH $3 \mathrm{~T} 3$ and HeLa are less than $\pm 0.3 \%$ (not shown). 


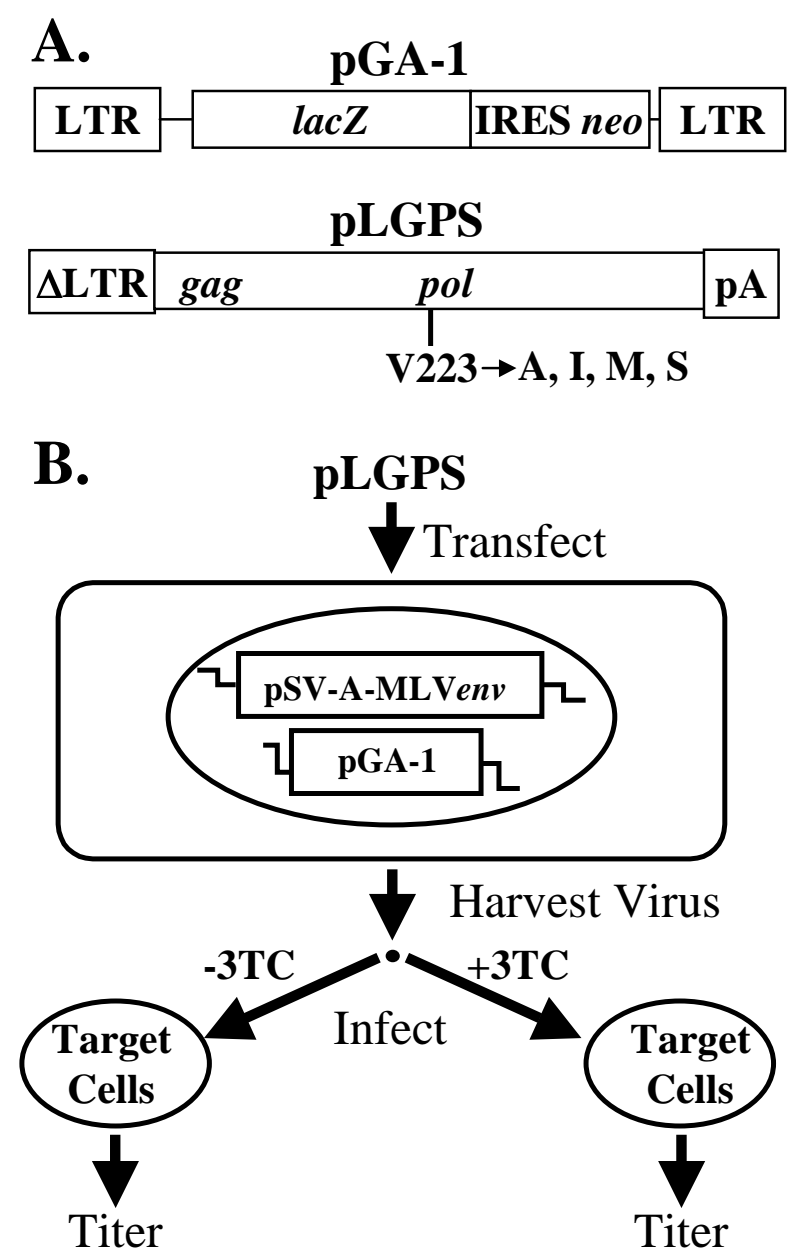

Figure 1

A.

\begin{tabular}{|l|l|l|} 
lacZ & IRES neo LTR \\
\hline
\end{tabular}

pLGPS

\begin{tabular}{|c|c|c|c|}
\hline$\Delta \mathrm{LTR}$ & gag & pol & $\mathbf{p A}$ \\
\hline
\end{tabular}

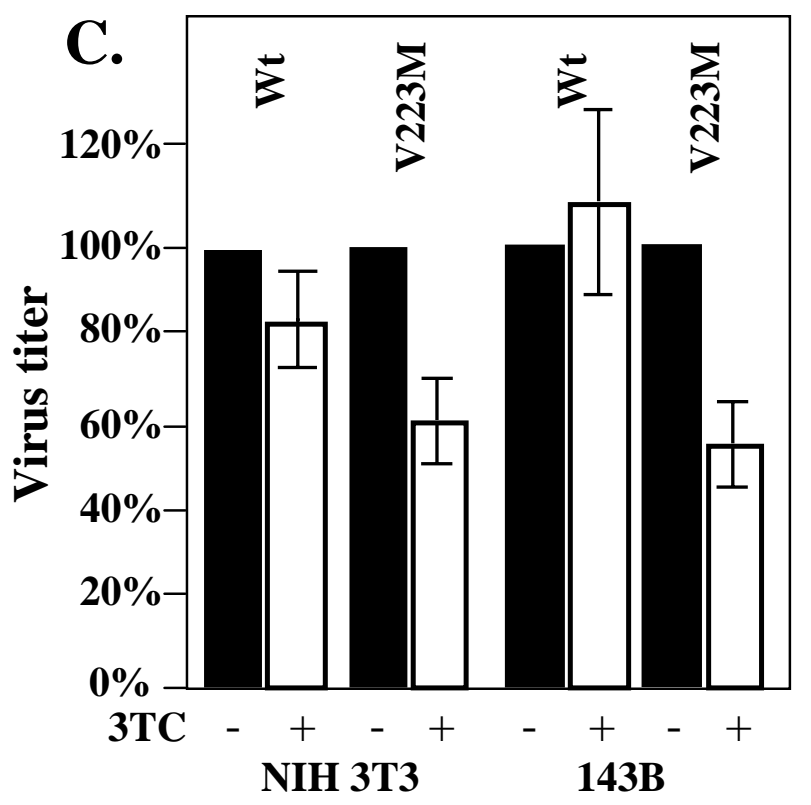


Figure 2
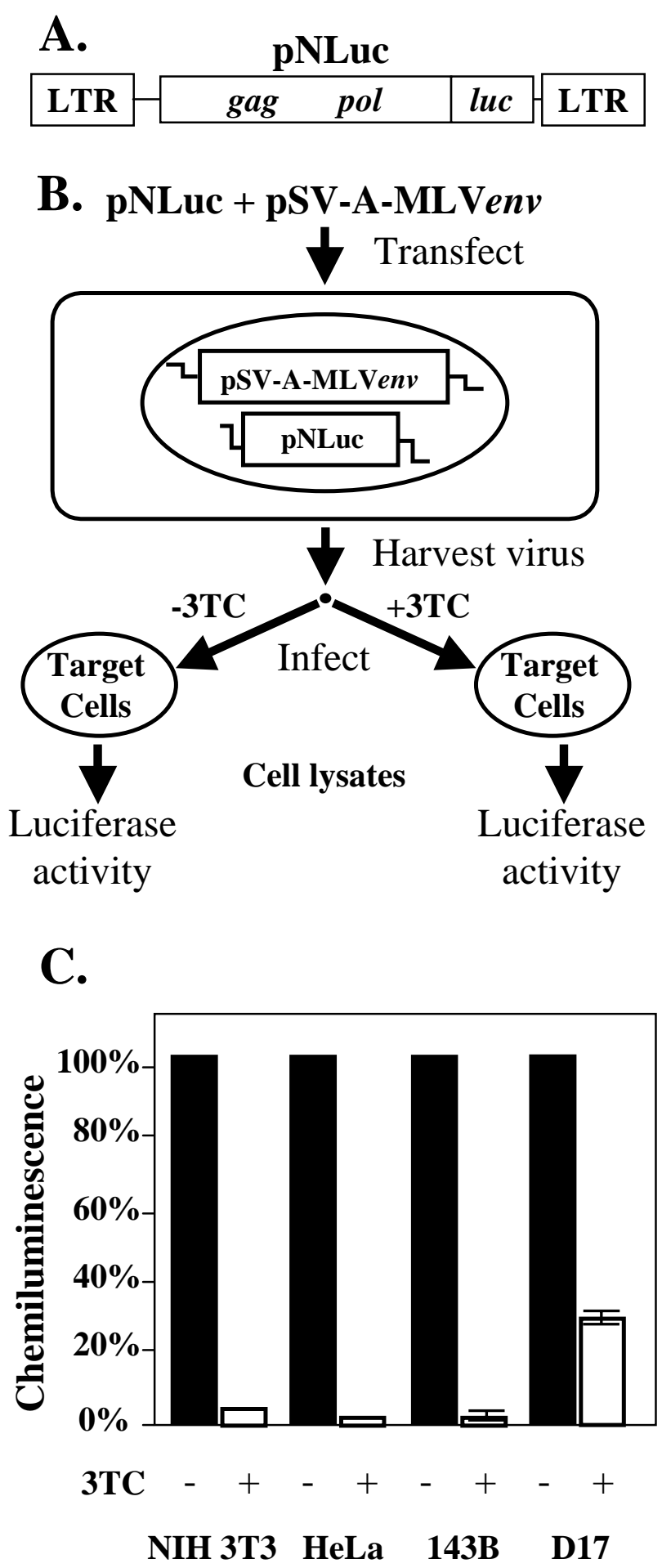


\section{REFERENCES}

1. Allen, M. I., M. Deslauriers, C. W. Andrews, G. A. Tipples, K. A. Walters, D. L. Tyrrell, N. Brown, and L. D. Condreay. 1998. Identification and characterization of mutations in hepatitis B virus resistant to lamivudine. Lamivudine Clinical Investigation Group. Hepatology 27:1670-1677.

2. Arts, E. J., and M. A. Wainberg. 1996. Mechanisms of nucleoside analog antiviral activity and resistance during human immunodeficiency virus reverse transcription. Antimicrob. Agents Chemother. 40:527-540.

3. Bakhanashvili, M., O. Avidan, and A. Hizi. 1996. Mutational studies of human immunodeficiency virus type 1 reverse transcriptase: the involvement of residues 183 and 184 in the fidelity of DNA synthesis. FEBS Lett. 391:257-262.

4. Balzarini, J., H. Pelemans, A. Karlsson, Q. E. De Clerc, and J. P. Kleim. 1996. Concomitant combination therapy for HIV infection preferable over sequential therapy with $3 \mathrm{TC}$ and non-nucleoside reverse transcriptase inhibitors. Proc. Natl. Acad. Sci. USA 93:13152-13157.

5. Boucher, C. A., N. Cammack, P. Schipper, R. Schuurman, P. Rouse, M. A. Wainberg, and J. M. Cameron. 1993. High-level resistance to (-) enantiomeric 2'-deoxy-3'-thiacytidine in vitro is due to one amino acid substitution in the catalytic site of human immunodeficiency virus type 1 reverse transcriptase. Antimicrob. Agents Chemother. 37:2231-2234. 
6. Boyer, P. L., and S. H. Hughes. 1995. Analysis of mutations at position 184 in reverse transcriptase of human immunodeficiency virus type 1. Antimicrob. Agents Chemother. 39:1624-1628.

7. Chang, C. N., S. L. Doong, J. H. Zhou, J. W. Beach, L. S. Jeong, C. K. Chu, C. H. Tsai, Y. C. Cheng, D. Liotta, and R. Schinazi. 1992. Deoxycytidine deaminase-resistant stereoisomer is the active form of (+/-)-2',3'-dideoxy-3'thiacytidine in the inhibition of hepatitis B virus replication [published erratum appears in J. Biol. Chem. 1992 Nov 25;267(33):24148]. J. Biol. Chem. 267:13938-13942.

8. Cherry, E., M. Slater, H. Salomon, E. Rud, and M. A. Wainberg. 1997. Mutations at codon 184 in simian immunodeficiency virus reverse transcriptase confer resistance to the (-) enantiomer of 2',3'-dideoxy- 3'-thiacytidine. Antimicrob. Agents Chemother. 41:2763-2765.

9. Coates, J. A., N. Cammack, H. J. Jenkinson, A. J. Jowett, M. I. Jowett, B. A. Pearson, C. R. Penn, P. L. Rouse, K. C. Viner, and J. M. Cameron. 1992. (-)2'-deoxy-3'-thiacytidine is a potent, highly selective inhibitor of human immunodeficiency virus type 1 and type 2 replication in vitro. Antimicrob. Agents Chemother. 36:733-739.

10. Coffin, J., M., S. H. Hughes, and H. E. Varmus. 1999. Retroviruses. Cold Spring Harbor Laboratory Press, Cold Spring Harbor, N.Y.

11. Cohen, J. 1997. The daunting challenge of keeping HIV suppressed [news]. Science 277:32-33. 
12. Cooley, T. P., L. M. Kunches, C. A. Saunders, J. K. Ritter, C. J. Perkins, C. McLaren, R. P. McCaffrey, and H. A. Liebman. 1990. Once-daily administration of 2',3'-dideoxyinosine (ddI) in patients with the acquired immunodeficiency syndrome or AIDS-related complex. Results of a Phase I trial. N. Engl. J. Med. 322:1340-1345.

13. Feng, J. Y., and K. S. Anderson. 1999. Mechanistic studies examining the efficiency and fidelity of DNA synthesis by the 3TC-resistant mutant (184V) of HIV-1 reverse transcriptase. Biochemistry 38:9440-9448.

14. Fischl, M. A., D. D. Richman, M. H. Grieco, M. S. Gottlieb, P. A. Volberding, O. L. Laskin, J. M. Leedom, J. E. Groopman, D. Mildvan, R. T. Schooley, G. G. Jackson, D. T. Durack, D. King and The AZT Collaborative Working Group. 1987. The efficacy of azidothymidine (AZT) in the treatment of patients with AIDS and AIDS-related complex. A double-blind, placebo-controlled trial. N. Engl. J. Med. 317:185-191.

15. Furman, P. A., M. Davis, D. C. Liotta, M. Paff, L. W. Frick, D. J. Nelson, R. E. Dornsife, J. A. Wurster, L. J. Wilson, J. A. Fyfe, J. V. Tuttle, W. H. Miller, L. Condreay, D. R. Averett, R. F. Schinazi, G. R. Painter. 1992. The antihepatitis B virus activities, cytotoxicities, and anabolic profiles of the (-) and (+) enantiomers of cis-5-fluoro-1-[2- (hydroxymethyl)-1,3-oxathiolan-5-yl]cytosine. Antimicrob. Agents Chemother. 36:2686-2692.

16. Gao, H.-Q., P. L. Boyer, S. G. Sarafianos, E. Arnold, and S. H. Hughes. Personal Communication. 
17. Gao, Q., Z. Gu, M. A. Parniak, J. Cameron, N. Cammack, C. Boucher, and M. A. Wainberg. 1993. The same mutation that encodes low-level human immunodeficiency virus type 1 resistance to 2',3'-dideoxyinosine and 2',3'dideoxycytidine confers high-level resistance to the (-) enantiomer of 2',3'dideoxy-3'- thiacytidine. Antimicrob. Agents Chemother. 37:1390-1392.

18. Georgiadis, M. M., S. M. Jessen, C. M. Ogata, A. Telesnitsky, S. P. Goff, and W. A. Hendrickson. 1995. Mechanistic implications from the structure of a catalytic fragment of Moloney murine leukemia virus reverse transcriptase. Structure 3:879-892.

19. Gu, Z., Q. Gao, H. Fang, H. Salomon, M. A. Parniak, E. Goldberg, J. Cameron, and M. A. Wainberg. 1994. Identification of a mutation at codon 65 in the IKKK motif of reverse transcriptase that encodes human immunodeficiency virus resistance to 2',3'-dideoxycytidine and 2',3'-dideoxy-3'-thiacytidine. Antimicrob. Agents Chemother. 38:275-281.

20. Gulick, R. M., J. W. Mellors, D. Havlir, J. J. Eron, C. Gonzalez, D. McMahon, L. Jonas, A. Meibohm, D. Holder, W. A. Schleif, J. H. Condra, E. A. Emini, R. Isaacs, J. A. Chodakewitz, and D. D. Richman. 1998. Simultaneous vs sequential initiation of therapy with indinavir, zidovudine, and lamivudine for HIV-1 infection: 100-week follow-up. JAMA 280:35-41.

21. Gulick, R. M., J. W. Mellors, D. Havlir, J. J. Eron, C. Gonzalez, D. McMahon, D. D. Richman, F. T. Valentine, L. Jonas, A. Meibohm, E. A. Emini, and J. A. Chodakewitz. 1997. Treatment with indinavir, zidovudine, and 
lamivudine in adults with human immunodeficiency virus infection and prior antiretroviral therapy. N. Engl. J. Med. 337:734-739.

22. Halvas, E. K., E. S. Svarovskaia, and V. K. Pathak. Unpublished Data.

23. Halvas, E. K., E. S. Svarovskaia, and V. K. Pathak. 2000. Development of an in vivo assay to identify structural determinants in murine leukemia virus reverse transcriptase important for fidelity. J. Virol. 74:312-319.

24. Hsiou, Y., J. Ding, K. Das, A. D. Clark, Jr., S. H. Hughes, and E. Arnold. 1996. Structure of unliganded HIV-1 reverse transcriptase at 2.7 A resolution: implications of conformational changes for polymerization and inhibition mechanisms. Structure 4:853-860.

25. Huang, H., R. Chopra, G. L. Verdine, and S. C. Harrison. 1998. Structure of a covalently trapped catalytic complex of HIV-1 reverse transcriptase: implications for drug resistance. Science 282:1669-1675.

26. Jang, S. K., H. G. Krausslich, M. J. Nicklin, G. M. Duke, A. C. Palmenberg, and E. Wimmer. 1988. A segment of the 5' nontranslated region of encephalomyocarditis virus RNA directs internal entry of ribosomes during in vitro translation. J. Virol. 62:2636-2643.

27. Kaushik, N., K. Singh, I. Alluru, and M. J. Modak. 1999. Tyrosine 222, a member of the YXDD motif of MuLV RT, is catalytically essential and is a major component of the fidelity center. Biochemistry 38:2617-2627.

28. Kawai, S., and M. Nishizawa. 1984. New procedure for DNA transfection with polycation and dimethyl sulfoxide. Mol. Cell. Biol. 4:1172-1174. 
29. Kemp, S. D., C. Shi, S. Bloor, P. R. Harrigan, J. W. Mellors, and B. A.

Larder. 1998. A novel polymorphism at codon 333 of human immunodeficiency virus type 1 reverse transcriptase can facilitate dual resistance to zidovudine and L-2',3'-dideoxy-3'-thiacytidine. J. Virol. 72:5093-5098.

30. Kent, R. B., J. R. Emanuel, Y. Ben Neriah, R. Levenson, and D. E. Housman. 1987. Ouabain resistance conferred by expression of the cDNA for a murine $\mathrm{Na}+$, K+-ATPase alpha subunit. Science 237:901-903.

31. Keulen, W., N. K. Back, A. van Wijk, C. A. Boucher, and B. Berkhout. 1997. Initial appearance of the 184Ile variant in lamivudine-treated patients is caused by the mutational bias of human immunodeficiency virus type 1 reverse transcriptase. J. Virol. 71:3346-3350.

32. Kiernan, R. E., and E. O. Freed. 1998. Cleavage of the murine leukemia virus transmembrane env protein by human immunodeficiency virus type 1 protease: transdominant inhibition by matrix mutations. J. Virol. 72:9621-9627.

33. Kiernan, R. E., A. Ono, G. Englund, and E. O. Freed. 1998. Role of matrix in an early postentry step in the human immunodeficiency virus type 1 life cycle. J. Virol. 72:4116-4126.

34. Kohlstaedt, L. A., J. Wang, J. M. Friedman, P. A. Rice, and T. A. Steitz. 1992. Crystal structure at 3.5 A resolution of HIV-1 reverse transcriptase complexed with an inhibitor. Science 256:1783-1790.

35. Krebs, R., U. Immendorfer, S. H. Thrall, B. M. Wohrl, and R. S. Goody. 1997. Single-step kinetics of HIV-1 reverse transcriptase mutants responsible for 
virus resistance to nucleoside inhibitors zidovudine and 3-TC. Biochemistry 36:10292-10300.

36. Kukhanova, M., S. H. Liu, D. Mozzherin, T. S. Lin, C. K. Chu, and Y. C. Cheng. 1995. L- and D-enantiomers of 2',3'-dideoxycytidine 5'-triphosphate analogs as substrates for human DNA polymerases. Implications for the mechanism of toxicity. J. Biol. Chem. 270:23055-23059.

37. Lafeuillade, A., C. Poggi, C. Tamalet, N. Profizi, C. Tourres, and O. Costes. 1997. Effects of a combination of zidovudine, didanosine, and lamivudine on primary human immunodeficiency virus type 1 infection. J. Infect. Dis. 175:10511055.

38. Landau, N. R., K. A. Page, and D. R. Littman. 1991. Pseudotyping with human T-cell leukemia virus type I broadens the human immunodeficiency virus host range. J. Virol. 65:162-169.

39. Larder, B. A., D. J. Purifoy, K. L. Powell, and G. Darby. 1987. Site-specific mutagenesis of AIDS virus reverse transcriptase. Nature 327:716-717.

40. Merigan, T. C., G. Skowron, S. A. Bozzette, D. Richman, R. Uttamchandani, M. Fischl, R. Schooley, M. Hirsch, W. Soo, C. Pettinelli, H. Schaumburg, and the ddC Study Group of the AIDS Clinical Trials Group. 1989. Circulating p24 antigen levels and responses to dideoxycytidine in human immunodeficiency virus (HIV) infections. A phase I and II study. Ann. Intern. Med. 110:189-194. 
41. Miller, A. D., J. V. Garcia, N. von Suhr, C. M. Lynch, C. Wilson, and M. V. Eiden. 1991. Construction and properties of retrovirus packaging cells based on gibbon ape leukemia virus. J. Virol. 65:2220-2224.

42. Murray, H. W., K. E. Squires, W. Weiss, S. Sledz, H. S. Sacks, J. Hassett, A. Cross, R. E. Anderson, and L. M. Dunkle. 1995. Stavudine in patients with AIDS and AIDS-related complex: AIDS clinical trials group 089. J. Infect. Dis. 171 Suppl 2:S123-130.

43. Poch, O., I. Sauvaget, M. Delarue, and N. Tordo. 1989. Identification of four conserved motifs among the RNA-dependent polymerase encoding elements. EMBO J. 8:3867-3874.

44. Powell, S. K., M. Artlip, M. Kaloss, S. Brazinski, R. Lyons, G. J. McGarrity, and E. Otto. 1999. Efficacy of antiretroviral agents against murine replicationcompetent retrovirus infection in human cells. J. Virol. 73:8813-8816.

45. Quan, Y., Z. Gu, X. Li, C. Liang, M. A. Parniak, and M. A. Wainberg. 1998. Endogenous reverse transcriptase assays reveal synergy between combinations of the M184V and other drug resistance-conferring mutations in interactions with nucleoside analog triphosphates. J. Mol. Biol. 277:237-247.

46. Rinaldo Jr, C. R., J. M. Liebmann, X. L. Huang, Z. Fan, Q. Al-Shboul, D. K. McMahon, R. D. Day, S. A. Riddler, and J. W. Mellors. 1999. Prolonged suppression of human immunodeficiency virus type 1 (HIV-1) viremia in persons with advanced disease results in enhancement of CD4 T cell reactivity to microbial antigens but not to HIV-1 antigens. J. Infect. Dis. 179:329-336. 
47. Sarafianos, S. G., K. Das, A. D. Clark, Jr., J. Ding, P. L. Boyer, S. H. Hughes, and E. Arnold. 1999. Lamivudine (3TC) resistance in HIV-1 reverse transcriptase involves steric hindrance with beta-branched amino acids. Proc. Natl. Acad. Sci. USA 96:10027-10032.

48. Schinazi, R. F., R. M. Lloyd, Jr., M. H. Nguyen, D. L. Cannon, A. McMillan, N. Ilksoy, C. K. Chu, D. C. Liotta, H. Z. Bazmi, and J. W. Mellors. 1993. Characterization of human immunodeficiency viruses resistant to oxathiolanecytosine nucleosides. Antimicrob. Agents Chemother. 37:875-881.

49. Smith, R. A., K. M. Remington, R. M. Lloyd, Jr., R. F. Schinazi, and T. W. North. 1997. A novel Met-to-Thr mutation in the YMDD motif of reverse transcriptase from feline immunodeficiency virus confers resistance to oxathiolane nucleosides. J. Virol. 71:2357-2362.

50. Smith, R. A., K. M. Remington, B. D. Preston, R. F. Schinazi, and T. W. North. 1998. A novel point mutation at position 156 of reverse transcriptase from feline immunodeficiency virus confers resistance to the combination of (-)-beta2',3'-dideoxy-3'-thiacytidine and 3'-azido-3'-deoxythymidine. J. Virol. 72:23352340.

51. Solder, B., U. Wintergerst, G. Notheis, J. Eberle, L. Gurtler, and B. H. Belohradsky. 1997. Effect of antiretroviral combination therapy (zidovudine/didanosine or zidovudine/lamivudine) on quantitative plasma human immunodeficiency virus-ribonucleic acid in children and adolescents infected with human immunodeficiency virus. J. Pediatr. 130:293-299. 
52. Stair, R. K., C. J. Nelson, and J. W. Mellors. 1991. Use of recombinant retroviruses to characterize the activity of antiretroviral compounds. J. Virol. 65:6339-6342.

53. Tantillo, C., J. Ding, A. Jacobo-Molina, R. G. Nanni, P. L. Boyer, S. H. Hughes, R. Pauwels, K. Andries, P. A. Janssen, and E. Arnold. 1994. Locations of anti-AIDS drug binding sites and resistance mutations in the threedimensional structure of HIV-1 reverse transcriptase. Implications for mechanisms of drug inhibition and resistance. J. Mol. Biol. 243:369-387.

54. Temesgen, Z., and A. J. Wright. 1999. Antiretrovirals. Mayo Clin Proc. 74:1284-1301.

55. Tisdale, M., S. D. Kemp, N. R. Parry, and B. A. Larder. 1993. Rapid in vitro selection of human immunodeficiency virus type 1 resistant to 3 '-thiacytidine inhibitors due to a mutation in the YMDD region of reverse transcriptase. Proc. Natl. Acad. Sci. USA 90:5653-5656.

56. Wilson, J. E., A. Aulabaugh, B. Caligan, S. McPherson, J. K. Wakefield, S. Jablonski, C. D. Morrow, J. E. Reardon, and P. A. Furman. 1996. Human immunodeficiency virus type-1 reverse transcriptase. Contribution of Met-184 to binding of nucleoside 5'-triphosphate. J. Biol. Chem. 271:13656-13662. 


\section{CHAPTER 5}

\section{DISCUSSION AND FUTURE DIRECTIONS}


Genetic variation of retroviruses. The retroviruses and variation in retroviral population were discovered at the same time (42). It has been observed that tumors, which resulted from infection by the same Rous sarcoma virus (RSV), possessed different morphology (18). These morphological variations in tumors were correlated with genetic differences of RSV providing evidence of high genetic variation in retroviral populations (51). Later, restriction digestion and sequencing techniques showed the existance of many closely related strains of MLV and spleen necrosis virus (SNV) (54). Additional evidence of retroviral genetic variation was the demonstration that passage of the same viral stock in tissue culture generated a pool of viruses with different RNase T1 fingerprints (15). A later work estimated the difference of the rate of nucleotide substitutions in a cellular gene versus viral genes. It has been concluded that retroviral genes evolve at a rate one million times faster than cellular genes (20). Now it appears that retroviruses are among the most diverse species in the world $(12-14,37)$.

It is important to study the mechanism that generate such a high genetic variation in retroviral populations, because it can provide evidence of how retroviruses escape the host immune response and develop drug resistance. It has been postulated genetic variation of retroviruses is a result of a combination of a high replication rate, rapid turnover of productively infected cells, and high mutation and recombination rates (1214, 37).

Mutation rates of retroviruses. Many approaches were used to measure the mutation rate of RTs. The most accurate way to estimate RT fidelity involves a forward mutation assay during single cycle of retroviral replication. The mutation rates of RTs differs for different retroviruses and range from 0.1 to $3 \times 10^{-5}$ mutations per base pair per 
replication cycle (37). For RSV, the mutation rate was measured by harvesting virus from cell clones and infecting fresh target cells (30). Viral RNA was analyzed by heteroduplex analysis. Mutations were detected by altered pattern of band migration on a gel. The mutation rate was estimated to be $1 \times 10^{-4}$ mutations per base pair per replication cycle. For MLV the mutation rate was measured by RNase T1oligonucleotide fingerprinting analysis of viral RNAs after one cycle of replication. It was estimated to be $2 \times 10^{-5}$ mutations per base pair per replication cycle. An elegant method was employed to measure the mutation rate for SNV replication. This method utilized a $L a c Z \alpha$ peptide gene as a reporter of mutations that could be screened by blue/white phenotype. The mutation rate was $1 \times 10^{-5}$ mutations per basepair per replication cycle $(38,39)$. Later, by utilizing the same approach the mutation rate was determined for HIV-1 to be $3 \times 10^{-5}$ mutations per basepair per replication cycle (33). Additionally, mutation rates of different mutant RTs, including drug-resistant mutants, were measured by different groups $(5,9,10,17,21-23,35,36,41,43,44,49$, 52, 53). Several in vitro studies indicated that some RT mutants displayed significantly higher fidelity than wild type the RT $(5,52,53,55)$. These mutants include the primer grip mutants, 3TC-resistant variants of HIV-1 RT, as well as the E89G mutant. This observation resulted in two hypotheses.

First, it was hypothesized that increased fidelity of 3TC-resistant variant of HIV-1 RT may account for lower emergence of virus in patients treated with 3TC, and for any delay of further resistance development for the other drugs (53). Based on this hypothesis it was proposed that it might be beneficial to select for M184V 3TC-resistant virus strain (with a high fidelity RT) in a patient prior to starting the treatment with other 
drugs. However, several investigators have been able to show that 3TC-resistant virus strains harboring the M184V mutation easily mutate and are selected for double-drug resistance when put under treatment with other drugs $(7,8,28)$. The speed of emergence of these double mutant viruses proved not to be significantly different from wild type viruses exposed to the same drugs. Also, the nature of mutations that were added to the M184V genetic background under drug treatment did not differ from those that were expected to appear in wild type virus under the same drug treatment. Taken together these data suggested that the 3TC resistant variant of HIV-1 RT possesses fidelity similar to that of the wild type. This result is in agreement with in vivo data from our laboratory, which indicate that the difference in fidelity between $\mathrm{M}$ and $\mathrm{V}$ variants of YVDD box of MLV RT is not more than two fold (22).

Second, it has been proposed that RTs evolve to possess a suboptimal fidelity (55). This suboptimal fidelity presumably is necessary for generation of retroviral genetic variation. The current studies provide evidence against the theory of suboptimal fidelity of RTs. We tested in vivo mutants of the primer grip that were previously shown to possess a higher fidelity in vitro. In our in vivo assay these mutants resulted in a significant reduction of the viral titers (Chapter 3), suggesting that primer grip variants that perhaps possess a higher fidelity were not selected as a wild type not because these mutants could not generate retroviral genetic variation, but because these primer grip mutants could not support viral replication. In addition, the overall data presented in the current study and other data from our laboratory indicated that among almost 100 MLV RT mutants generated, none of them displayed fidelity more than $30 \%$ higher than wild type RT, suggesting that wild type RTs possesses an optimal fidelity. However, it is 
possible that we just did not make a mutation in MLV RT that could increase the accuracy of the DNA synthesis.

To address this possibility we are planning to develop an in vivo assay for selection of MLV and HIV-1 RT variants that have a higher fidelity than wild type (Fig. 1). First, we will construct a retroviral vector that will encode for gag-pol proteins, as well as GFP-hygro fusion protein. This retroviral vector will contain all cis elements necessary for retroviral replication including a packaging signal $\psi$. The sequence of the pol gene that codes for RT will be randomized by PCR to ensure the presence of all possible RT mutants. Second, we will construct two cell lines expressing two different envelopes. The envelopes will be selected in such a way that self-reinfection of both cell lines will be prevented and virus produced by one cell line will be able to infect the other. The retroviral vector containing gag-pol and GFP-hygro genes will be infected at a low multiplicity of infection into one of the cell lines. The low multiplicity of infection is necessary to ensure that each virus that will be produced from these infected cells will contain RT and a RNA genome transcribed from the same provirus. Virus collected from the infected cell (infected cells will be sorted by FACS to select for infected cells that can produce virus to increase viral titer) and will be used to infect the other cell line. Pingpong infections of fresh (uninfected) cells lines will be repeated several times and then followed by hygro selection and FACS to sort out the GFP positive cells. A ping-pong infection is procedure in which two cell lines that express different viral envelope proteins and cocultivated. As a result virus produced from each cell line effitiently infect the other cell line. This procedure will allow us to select for RT mutants that did not introduce any inactivating mutations into GFP-hygro gene. The overall procedure will be 
repeated several times until mutant RT variants that possess a higher fidelity will appear. Sequencing the RT gene will reveal the genotype of this mutant(s). A similar approach that employs a reversion mutation will be used to select for a mutant RT with a lower fidelity.

Recombination of retroviruses. Recombination of retroviruses also occurs at a high rate and contributes to the generation of genetic variation of retroviruses $(1,24,25,31$, 50). Recombination rates of retroviruses have been measured by utilizing single cycle replication assays and were estimated to be around $4 \%$ per $1 \mathrm{~kb}$ per replication cycle (2). Although we did not measure the rate of recombination in this study, our data indicate that the rate of template switching events (including recombination) may be altered significantly by different factors (chapter 2). One of the factors that could affect the frequency of template switching is the intracellular conditions such as presence of HU. The presence of HU altered the dNTP concentration in the cytoplasm and as a result decreased rate of DNA polymerization during reverse transcription (40). The decreased rate of DNA polymerization caused the frequency of template switching to go up by approximately 2 -fold. These data should be taken in consideration when one proposes HU as an anti-retroviral agent. Another factor that resulted in 5-fold increase in frequency of template switching is a single point mutation in the RT (chapter 1). These data bring up an intriguing possibility that similar mutants play a role in generating genetic variation of retroviruses during the natural course of the retroviral replication. If these mutation arise in viral populations, they may undergo extensive recombination and generate complex recombination. More experimental evidence is required for investigating this possibility. 
Previously, two models were proposed for retroviral recombination: forced copychoice model and strand displacement model $(13,26,45)$. Recent studies have favored the forced-copy choice model for retroviral recombination (2). The forced copy-choice model proposes that a break in the viral RNA will force the RT to choose the other copy of the RNA genome and result in a recombination event. In the current study we modified this model. The new dynamic copy-choice model suggests that the driving force for switching templates is the formation of 3' homology between newly synthesized DNA and the other copy of RNA (chapter 2, Fig. 6 in chapter 2).

Interestingly, the same model of template switching might be applied for the minus and plus strand transfer events. If we assume that the RNA genome forms a structure inside of the viral core that by some reason brings two $\mathrm{R}$ regions together, the minus strand transfer event completely matches the proposed model for template switching (Fig. 2). The homology of the R regions are necessary for formation of the 3' homology between minus strand strong stop DNA and R region of the 3' end of viral RNA. This mechanism would suggest that there is no "JUMP" between the two ends of the viral RNA. It is not clear if the disassociation of the tRNA from PBS takes place after minus strand transfer, however it would not be required for this model of strand transfer. Even more, the tRNA-PBS interactions might be necessary to hold the ends of RNA(DNA) template together for providing the plus strand transfer. Again, the plus strand transfer may undergo the same mechanism of template switching (Fig. 2). The only concern for the plus strand transfer is that length of homology for this template switch is as short as 18 nucleotides. It is not known if these 18 nucleotides that are 
associated with RT are available for interaction with the homologous sequence to form the 3 ' interactions which were proposed to drive the switch.

It has been very well established that the RNase $\mathrm{H}$ activity is important for strand transfer events in vivo and in vitro. The in vivo the requirement for the RNase $\mathrm{H}$ activity was only demonstrated for the minus and plus strand transfer events $(46,48)$. We demonstrate by the data presented in chapter 2 that RNase $\mathrm{H}$ activity is also important for other than minus and plus strand transfers in vivo, suggesting a similar mechanism of all template switches. Future experiments are required to investigate whether polymerization dependent or polymerization independent (or may be both) RNase H activities play a role in template switching and recombination.

The dynamic copy choice model was proposed by an interpretation that the dNTP-binding site mutants of MLV RT possess a slower polymerization rate than wild type RT, however it never was demonstrated directly. Perhaps a real time PCR technique will help us to support this assumption. Additionally, the role of other viral proteins, such as NC, for the frequency of the template switching could be tested by employing the assay developed in this study (chapter 2).

Mechanism of fidelity of RT and other polymerases. DNA polymerases make an error only once per $10^{4}-10^{5}$ initial nucleotide incretions during DNA replication (34). It is still not well understood how DNA polymerases select for incorporation of a correct base with such a high fidelity. Both geometric effects and base-base hydrogen bonding have been thought to be important factors in DNA synthesis fidelity $(19,27,32)$. However, recently it has been demonstrated that formation of hydrogen bonds between incoming dNTP and a corresponding template base is not absolutely necessary for incorporation of 
a correct dNTP (34). The authors showed the analogs of dNTPs that possessed a similar shape but were not able to form hydrogen bonds could be successfully incorporated into DNA in accordance to their shape.

Two other recent studies have also provided interesting information about mechanisms of fidelity by DNA polymerases. In these studies the crystal structures of T7 DNA polymerase and Bacillus DNA polymerase were solved in a complex with template-primer $(16,29)$. Both structures provided details of a sequence-independent molecular recognition of correctly formed base pairs. Protein side-chains have been shown to interact in the minor groove of the first four base pairs extending from the 3' primer terminus. These highly conserved protein side chains or oriented water molecules anchored to protein side chain form hydrogen bonds to the N3 position of purine bases and $\mathrm{O} 2$ position of pyrimidine bases $(16,29,47)$. These interactions can occur only with correct Watson-Crick base pairs. A mismatch could not bind with the same geometry as a correctly formed base-pair, resulting in misalignment of the 3'-OH primer terminus, thereby preventing incorporation of the next nucleotide. As a consequence, the proofreading activity of these enzymes would remove an incorrect base and then continue the DNA synthesis. At first glance, this mechanism of fidelity is not related to RT, because it was thought for a long time that RTs do not possess the proofreading. However, the AZT-resistance mechanism indicated that HIV-1 RT is able to remove the incorporated base (see details in chapter 1), suggesting that similar mechanism of fidelity could be considered $(3,4)$.

In chapter 3, we discuss how mutations of the minor groove binding helix affect the fidelity of the MLV RT. Interestingly, the amino acids that we mutated are 
contacting the second, third, and fourth nucleotide upstream of the 3' $\mathrm{OH}$ of the primer (Fig. 6 in chapter 3). Even though these amino acids do not contact the $\mathrm{O} 2$ and the N3 positions of the nucleotide bases, mutations at these positions do affect fidelity up to 2.8fold. The mechanism by which these residues of the thumb domain can influence the fidelity of RT remains to be determined.

New approaches to overcome viral replication and drug-resistance. Development of drug resistance depends on the presence of viruses that possess drug resistant mutations in the viral population at the time of the beginning of drug treatment $(12,14)$. It would be interesting to identify the inhibitors of the drug-resistant HIV-1 RTs. The easiest way would be to find an inhibitor to the 3TC-resistant HIV-1 RT, because resistance to 3TC is always associated with mutation at a single residue M184 of the YMDD motif (6). A similar approach that was used for identifying the inhibitors of wild-type RT by using a massive screening of large number of compounds by relatively simple and possibly automated RT activity assays may be employed for identification of inhibitors of 3TCresistant RTs. Once an inhibitor is found it could be introduced before (if a combination of inhibitors will result in a higher toxicity problem) or at the same time (if toxicity would not be a consideration) with 3TC. Perhaps, introducing the new inhibitor of the 3TC- resistant RT will slow down the development of 3TC resistance, because the number of drug resistant viruses that could replicate will be significantly reduced. Additionally, identification of an inhibitor against the drug-resistant variants may be beneficial for those HIV-1 infected patients that already carry the drug-resistant population as a result of monotherapy with 3TC. In this situation, the introduction of other drugs targeted against different sites of RT or protease will not be useful because 
the M184V viral population already contains all other mutations and is ready for development a new second drug resistance. The approach of looking for drugs that can slow down the rate of infecting new cells (such as anti RT or protease) are important because they were shown to be able to reduce viral load to undetectable levels, and as a result, increase CD4 positive cell counts and improve the overall health of the patient. However, these drugs do not provide a long-term solution to the problem. It was shown that even after a ten-year period of undetectable plasma virus, removal of the drug resulted in reemergence of the virus. This virus seems to be a wild type (not drugresistant) virus that is probably present in a form of provirus in a long living cell reservoir. It is not clear what kinds of cells constitute these reservoirs and what is the half-life time of cells.

A different kind of approach perhaps could be utilized to address this problem. We do not have to wait for cells that carry a proviral DNA inserted in the cellular genome to die, but we can try to selectively kill these cells. For example, a gene therapy approach could be employed. We can create a virus that is pseudotyped with CD4 and CCR5 proteins. Presumably, this virus would be able to selectively infect cells that express the HIV-1 envelope proteins. This virus could carry a retroviral vector that contains a DTA gene that is present in two separated fragments in LTRs. Reverse transcription will result in reconstitution of the toxic gene and expresson of the toxic protein (11). The cells that contain the proviral DNA will be selectively killed and the virus-containing reservoir will be eliminated. 


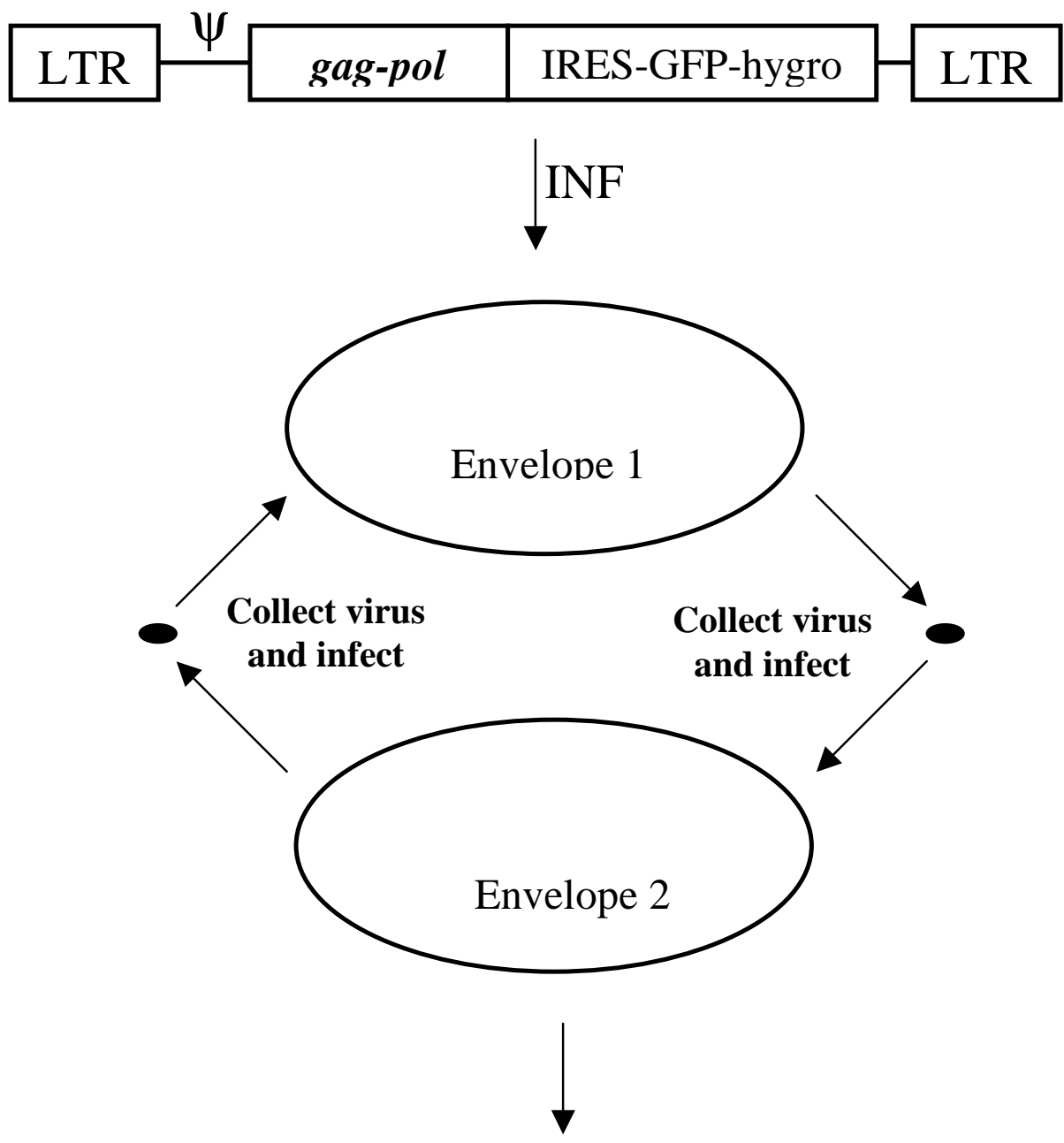

Hygro selection and FACS

Figure 1. A schematic representation of in vivo assay to select for RT with a high fidelity. 

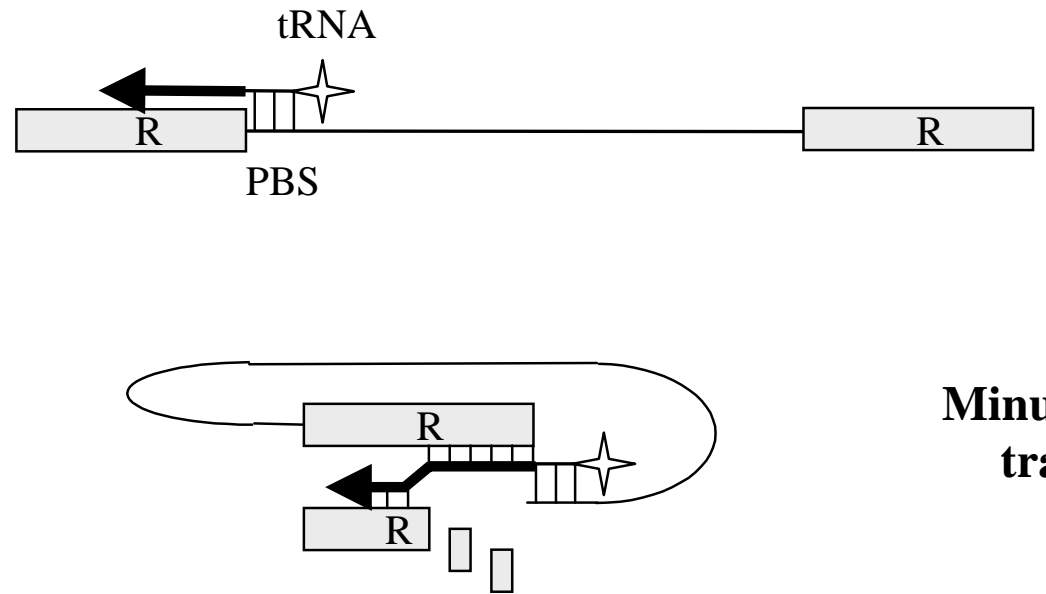

Minus-strand transfer

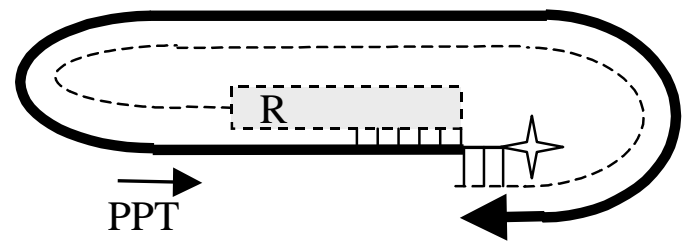

\author{
Minus-strand \\ synthesis
}

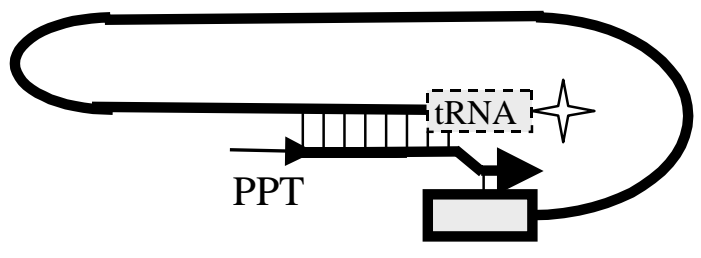

\title{
Plus-strand transfer
}

Figure 2. Template switching model for minus-strand and plus-strand transfers. Thin lane represents RNA; bold lane represents DNA; dashed lanes represent RNase H degradation. Repeated sequences are shown as boxes. 


\section{REFERENCES}

1. Alevy, M. C., and P. K. Vogt. 1978. Ts pol mutants of avian sarcoma viruses: mapping and demonstration of single cycle recombinants. Virology 87:21-33.

2. Anderson, J. A., E. H. Bowman, and W. S. Hu. 1998. Retroviral recombination rates do not increase linearly with marker distance and are limited by the size of the recombining subpopulation. J Virol 72:1195-1202.

3. Arion, D., N. Kaushik, S. McCormick, G. Borkow, and M. A. Parniak. 1998. Phenotypic mechanism of HIV-1 resistance to 3'-azido-3'-deoxythymidine (AZT): increased polymerization processivity and enhanced sensitivity to pyrophosphate of the mutant viral reverse transcriptase. Biochemistry 37:15908-15917.

4. Arion, D., N. Sluis-Cremer, and M. A. Parniak. 2000. Mechanism by which phosphonoformic acid resistance mutations restore 3'- azido-3'-deoxythymidine (AZT) sensitivity to AZT-resistant HIV-1 reverse transcriptase. J Biol Chem 275:9251-9255.

5. Bakhanashvili, M., O. Avidan, and A. Hizi. 1996. Mutational studies of human immunodeficiency virus type 1 reverse transcriptase: the involvement of residues 183 and 184 in the fidelity of DNA synthesis. FEBS Lett 391:257-262.

6. Balzarini, J. 1999. Suppression of resistance to drugs targeted to human immunodeficiency virus reverse transcriptase by combination therapy. Biochem Pharmacol 58:1-27.

7. Balzarini, J., H. Pelemans, E. De Clercq, A. Karlsson, and J. P. Kleim. 1997. Reverse transcriptase fidelity and HIV-1 variation. Science 275:229-230; discussion 230-221. 
8. Balzarini, J., H. Pelemans, M. J. Perez-Perez, A. San-Felix, M. J. Camarasa, E. De Clercq, and A. Karlsson. 1996. Marked inhibitory activity of nonnucleoside reverse transcriptase inhibitors against human immunodeficiency virus type 1 when combined with (-)2',3'-dideoxy-3'-thiacytidine. Mol Pharmacol 49:882-890.

9. Beard, W. A., S. J. Stahl, H. R. Kim, K. Bebenek, A. Kumar, M. P. Strub, S. P. Becerra, T. A. Kunkel, and S. H. Wilson. 1994. Structure/function studies of human immunodeficiency virus type 1 reverse transcriptase. Alanine scanning mutagenesis of an alpha-helix in the thumb subdomain. J Biol Chem 269:2809128097.

10. Bebenek, K., W. A. Beard, J. R. Casas-Finet, H. R. Kim, T. A. Darden, S. H. Wilson, and T. A. Kunkel. 1995. Reduced frameshift fidelity and processivity of HIV-1 reverse transcriptase mutants containing alanine substitutions in helix $\mathrm{H}$ of the thumb subdomain. J Biol Chem 270:19516-19523.

11. Cheslock, S., J. Anderson, C. Hwang, V. K. Pathak, and W.-S. Hu. 2000. Submitted to J. Virol. .

12. Coffin, J. M. 1995. HIV population dynamics in vivo: implications for genetic variation, pathogenesis, and therapy. Science 267:483-489.

13. Coffin, J. M. 1979. Structure, replication, and recombination of retrovirus genomes: some unifying hypotheses. J Gen Virol 42:1-26.

14. Coffin, J. M., S. H. Hughes, and H. E. Varmus. 1997. Retroviruses. Cold Spring Harbor Laboratory Press, Cold Spring Harbor, NY. 
15. Coffin, J. M., P. N. Tsichlis, C. S. Barker, S. Voynow, and H. L. Robinson. 1980. Variation in avian retrovirus genomes. Ann N Y Acad Sci 354:410-425.

16. Doublie, S., S. Tabor, A. M. Long, C. C. Richardson, and T. Ellenberger. 1998. Crystal structure of a bacteriophage T7 DNA replication complex at $2.2 \mathrm{~A}$ resolution [see comments]. Nature 391:251-258.

17. Drosopoulos, W. C., and V. R. Prasad. 1996. Increased polymerase fidelity of E89G, a nucleoside analog-resistant variant of human immunodeficiency virus type 1 reverse transcriptase. J Virol 70:4834-4838.

18. Duran-Reynals, F. 1942. The reciprocal infections of ducks and chickens with tumor-inducing viruses. Cancer Research 2:343-369.

19. Echols, H., and M. F. Goodman. 1991. Fidelity mechanisms in DNA replication. Annu Rev Biochem 60:477-511.

20. Gojobori, T., and S. Yokoyama. 1985. Rates of evolution of the retroviral oncogene of Moloney murine sarcoma virus and of its cellular homologues. Proc Natl Acad Sci U S A 82:4198-4201.

21. Gutierrez-Rivas, M., A. Ibanez, M. A. Martinez, E. Domingo, and L. Menendez-Arias. 1999. Mutational analysis of Phe160 within the "palm" subdomain of human immunodeficiency virus type 1 reverse transcriptase. J Mol Biol 290:615-625.

22. Halvas, E. K., E. S. Svarovskaia, and V. K. Pathak. 2000. Development of an in vivo assay to identify structural determinants in murine leukemia virus reverse transcriptase important for fidelity. J Virol 74:312-319. 
23. Halvas, E. K., E. S. Svarovskaia, and V. K. Pathak. 2000. The role of murine leukemia virus reverse transcriptase dNTP-binding site in retroviral replication and in vivo fidelity (submitted to J.Virol.).

24. Hu, W. S., and H. M. Temin. 1990. Genetic consequences of packaging two RNA genomes in one retroviral particle: pseudodiploidy and high rate of genetic recombination. Proc Natl Acad Sci U S A 87:1556-1560.

25. Hu, W. S., and H. M. Temin. 1990. Retroviral recombination and reverse transcription. Science 250:1227-1233.

26. Hunter, E. 1978. The mechanism for genetic recombination in the avian retroviruses. Curr Top Microbiol Immunol 79:295-309.

27. Joyce, T., V. Kenny, K. McGuigan, and J. Barnes. 1992. Disinfection of water by sunlight [published erratum appears in Lancet 1992 Nov 28;340(8831):1362]. Lancet 340:921.

28. Keulen, W., M. Nijhuis, R. Schuurman, B. Berkhout, and C. Boucher. 1997. Reverse transcriptase fidelity and HIV-1 variation [letter; comment]. Science 275:229; discussion 230-221.

29. Kiefer, J. R., C. Mao, J. C. Braman, and L. S. Beese. 1998. Visualizing DNA replication in a catalytically active Bacillus DNA polymerase crystal [see comments]. Nature 391:304-307.

30. Leider, J. M., P. Palese, and F. I. Smith. 1988. Determination of the mutation rate of a retrovirus. J Virol 62:3084-3091.

31. Linial, M., and S. Brown. 1979. High-frequency recombination within the gag gene of Rous sarcoma virus. J Virol 31:257-260. 
32. Loeb, L. A., and T. A. Kunkel. 1982. Fidelity of DNA synthesis. Annu Rev Biochem 51:429-457.

33. Mansky, L. M., and H. M. Temin. 1995. Lower in vivo mutation rate of human immunodeficiency virus type 1 than that predicted from the fidelity of purified reverse transcriptase. J Virol 69:5087-5094.

34. Morales, J. C., and E. T. Kool. 1998. Efficient replication between nonhydrogen-bonded nucleoside shape analogs. Nat Struct Biol 5:950-954.

35. Nijhuis, M., R. Schuurman, D. de Jong, R. van Leeuwen, J. Lange, S. Danner, W. Keulen, T. de Groot, and C. A. Boucher. 1997. Lamivudineresistant human immunodeficiency virus type 1 variants $(184 \mathrm{~V})$ require multiple amino acid changes to become co-resistant to zidovudine in vivo. J Infect Dis 176:398-405.

36. Oude Essink, B. B., N. K. Back, and B. Berkhout. 1997. Increased polymerase fidelity of the 3TC-resistant variants of HIV-1 reverse transcriptase. Nucleic Acids Res 25:3212-3217.

37. Pathak, V. K., and W. S. Hu. 1997. "Might as well jump!" Template switching by retroviral reverse transcriptase, defective genome formation, and recombination. Seminars in virology 8:141-150.

38. Pathak, V. K., and H. M. Temin. 1990. Broad spectrum of in vivo forward mutations, hypermutations, and mutational hotspots in a retroviral shuttle vector after a single replication cycle: deletions and deletions with insertions. Proc Natl Acad Sci U S A 87:6024-6028. 
39. Pathak, V. K., and H. M. Temin. 1990. Broad spectrum of in vivo forward mutations, hypermutations, and mutational hotspots in a retroviral shuttle vector after a single replication cycle: substitutions, frameshifts, and hypermutations. Proc Natl Acad Sci U S A 87:6019-6023.

40. Pfeiffer, J. K., R. S. Topping, N. H. Shin, and A. Telesnitsky. 1999. Altering the intracellular environment increases the frequency of tandem repeat deletion during Moloney murine leukemia virus reverse transcription. J Virol 73:84418447.

41. Roberts, J. D., B. D. Preston, L. A. Johnston, A. Soni, L. A. Loeb, and T. A. Kunkel. 1989. Fidelity of two retroviral reverse transcriptases during DNAdependent DNA synthesis in vitro. Mol Cell Biol 9:469-476.

42. Rous, P., and J. B. Murphy. 1913. Variation in chiken sarcoma caused by filterable agent. J. Exper. Med. 17:219-231.

43. Rubinek, T., M. Bakhanashvili, R. Taube, O. Avidan, and A. Hizi. 1997. The fidelity of 3' misinsertion and mispair extension during DNA synthesis exhibited by two drug-resistant mutants of the reverse transcriptase of human immunodeficiency virus type 1 with Leu74-->Val and Glu89-->Gly. Eur J Biochem 247:238-247.

44. Sarafianos, S. G., V. N. Pandey, N. Kaushik, and M. J. Modak. 1995. Glutamine 151 participates in the substrate dNTP binding function of HIV-1 reverse transcriptase. Biochemistry 34:7207-7216.

45. Skalka, A. M., L. Boone, R. Junghans, and D. Luk. 1982. Genetic recombination in avian retroviruses. J Cell Biochem 19:293-304. 
46. Smith, C. M., J. S. Smith, and M. J. Roth. 1999. RNase H requirements for the second strand transfer reaction of human immunodeficiency virus type 1 reverse transcription. J Virol 73:6573-6581.

47. Steitz, T. A. 1998. A mechanism for all polymerases. Nature 391:231-232.

48. Tanese, N., A. Telesnitsky, and S. P. Goff. 1991. Abortive reverse transcription by mutants of Moloney murine leukemia virus deficient in the reverse transcriptase-associated RNase H function. J Virol 65:4387-4397.

49. Taube, R., O. Avidan, and A. Hizi. 1997. The fidelity of misinsertion and mispair extension throughout DNA synthesis exhibited by mutants of the reverse transcriptase of human immunodeficiency virus type 2 resistant to nucleoside analogs. Eur J Biochem 250:106-114.

50. Temin, H. M. 1991. Sex and recombination in retroviruses. Trends Genet 7:7174.

51. Temin, H. M., and H. Rubin. 1958. Characteristics of an assay for Rous sarcoma virus and Rous sarcoma cells in tissue culture. Virol. 6:669-688.

52. Wainberg, M. A. 1997. Increased fidelity of drug-selected M184V mutated HIV1 reverse transcriptase as the basis for the effectiveness of 3TC in HIV clinical trials. Leukemia 11 Suppl 3:85-88.

53. Wainberg, M. A., W. C. Drosopoulos, H. Salomon, M. Hsu, G. Borkow, M. Parniak, Z. Gu, Q. Song, J. Manne, S. Islam, G. Castriota, and V. R. Prasad. 1996. Enhanced fidelity of 3TC-selected mutant HIV-1 reverse transcriptase. Science 271:1282-1285. 
54. Weiss, R., N. Teich, H. Varmus, and J. Coffin. 1985. RNA tumor viruses, 2-nd ed. Cold Sping Harbor Laboratory, Cold Sping Harbor Laboratory, NY.

55. Wisniewski, M., C. Palaniappan, Z. Fu, S. F. Le Grice, P. Fay, and R. A.

Bambara. 1999. Mutations in the primer grip region of HIV reverse transcriptase can increase replication fidelity. J Biol Chem 274:28175-28184. 


\section{APPENDIX 1}

FACS ANALYSIS DATA FOR POL + RNASE H DOUBLE MUTANTS, AND F156W AND RNASE H MUTANTS IN THE PRESENCE AND ABSENCE OF HYDROXYUREA 
To test the dynamic-copy choice model for retroviral recombination proposed in Chapter 2, we performed additional analysis of MLV RT mutants. First, we constructed double mutants containing mutations in the Pol as well as RNase H domains. The V223I mutation in the active site of the Pol domain that increased the frequency of template switching by two-fold was combined with the Y598V RNase H mutant that decreased the frequency of template switching. The V223I/Y598V double mutant exhibited the frequency of template switching similar to the RNase H mutant Y598V (Table 1 and Fig. 1). This result suggested that slowing down the rate of polymerization by approximately two-fold was not sufficient to rescue the RNase H defect of the Y598V mutant. This result was consistent with previously observed results that slowing down polymerization by HU by approximately two-fold also did not rescue the defect of this RNase H mutant (Table 2, and Chapter 2). We also constructed a double mutant that contained the F156W mutation in the Pol domain as well as Y598V mutation in the RNase H domain. This mutant also did not show a statically significant effect on the frequency of template switching in comparison to the Y598V mutant (Table1 and Figure 1). This result suggested that further slowing down the rate of polymerization still was not sufficient for rescuing the RNase $\mathrm{H}$ mutation defect. Perhaps, the Y598V mutant possesses such a low RNase $\mathrm{H}$ activity that all direct repeat deletions occur during the plus-strand DNA synthesis.

To investigate whether any of the RNase $\mathrm{H}$ mutants could be rescued by the slowing down the rate of polymerization we performed the direct repeat deletion assay for the S526A and R657S RNase H mutants in the presence of HU. The frequency of 
template switching was not changed for the S526A mutant in the presence of HU, suggesting that HU did not rescue the defect of the S526A mutant (Table 2 and Figure 2). Interestingly, the R657S RNase H mutant displayed an increased frequency of template switching in the presence of HU (Table 2 and Figure 2). This observation supported the dynamic copy-choice model proposed in Chapter 2.

Additionally, we tested the frequency of template switching of the F156W mutant in the presence of $\mathrm{HU}$ (Table 2 and Figure 2). In presence of HU, the F156W mutant did not display a higher frequency of template switching than that observed previously in Chapter 2, suggesting that slower dNTP-binding in the presence of HU due to decreasing the nucleotide pool is not a rate limiting step in the case of F156W mutant. It is possible that in case of the F156W mutant a step in catalysis that subsequent to the substrate dNTP binding is rate limiting. Surprisingly, the frequency of template switching by the F156W mutant in the absence of HU was higher than previously observed in Chapter 2. This result could be explained by the fact that higher titers were obtained in the current experiment, which could produce a significant proportion of double-infected target cells and artificially increased the percent of the GFP positive cells. 
TABLE 1. Effect of mutations in MLV RT on the frequency of direct repeat deletion and GFP reconstitution

\begin{tabular}{|c|c|c|c|c|}
\hline $\begin{array}{c}\text { RT } \\
\text { mutants }\end{array}$ & $\begin{array}{c}\text { No. of } \\
\text { experiments }\end{array}$ & $\begin{array}{l}\text { Total no. of } \\
\text { colonies }^{a}\end{array}$ & $\begin{array}{c}\text { Frequency of } \\
\text { direct repeat } \\
\text { deletions } \\
(\text { mean } \% \pm \mathrm{SE})^{b}\end{array}$ & $\begin{array}{c}\text { Relative change } \\
\text { in direct repeat } \\
\text { deletion } \\
\text { frequency }^{c}\end{array}$ \\
\hline Wild type & 5 & $>3000$ & $12.1 \pm 0.9$ & 1.0 \\
\hline Y598V & 3 & $>1500$ & $6.4 \pm 0.2$ & 0.5 \\
\hline V223I & 3 & $>3000$ & $30.5 \pm 1.6$ & 2.5 \\
\hline F156W & 5 & $>5000$ & $73.2 \pm 2.2$ & 6.0 \\
\hline V223I / Y598V & 3 & $>1500$ & $5.2 \pm 0.5$ & 0.4 \\
\hline F156W / Y598V & 3 & $>1500$ & $9.2 \pm 1.9$ & 0.7 \\
\hline \multicolumn{5}{|c|}{${ }^{b}$ Frequency of direct repeat deletion was determined as a percent of infected D17 } \\
\hline \multicolumn{5}{|c|}{ target cells that exhibited fluorescence after G418 selection compared to the negative } \\
\hline \multicolumn{5}{|c|}{ control. The standard error of the mean (SE) was determined by using the Sigma Plot 5.0 } \\
\hline \multicolumn{5}{|l|}{ program. } \\
\hline \multicolumn{5}{|c|}{${ }^{c}$ Calculated as follows: frequency of direct repeat deletion observed with mutant } \\
\hline \multicolumn{5}{|c|}{ MLV RT $\div$ frequency of direct repeat deletion observed with the wild-type RT. } \\
\hline Statistically signif & ant changes in $t$ & frequency of $\mathrm{c}$ & ect repeat deletion & $\mathrm{s}$, relative to the \\
\hline
\end{tabular}


TABLE 2. Effect of HU treatment on the frequency of direct repeat deletion and GFP reconstitution

\begin{tabular}{|c|c|c|c|c|}
\hline $\begin{array}{l}\text { MLV RT } \\
\text { mutants }\end{array}$ & $\begin{array}{c}\text { No. of } \\
\text { experiments }\end{array}$ & $\begin{array}{l}\text { Total no. of } \\
\text { colonies }^{a}\end{array}$ & $\begin{array}{c}\text { Frequency of } \\
\text { direct repeat } \\
\text { deletions } \\
(\text { mean } \% \pm \mathrm{SE})^{b}\end{array}$ & $\begin{array}{c}\text { Relative change } \\
\text { in direct repeat } \\
\text { deletion } \\
\text { frequency }{ }^{c}\end{array}$ \\
\hline Wild type & 5 & $>3000$ & $12.1 \pm 0.9$ & 1.0 \\
\hline Wild Type + HU & 4 & $>2000$ & $31.2 \pm 0.8$ & 2.5 \\
\hline Y598V & 3 & $>1500$ & $6.4 \pm 0.2$ & 1.0 \\
\hline Y598V + HU & 3 & $>450$ & $3.9 \pm 0.8$ & none \\
\hline S256A & 3 & $>4000$ & $6.1 \pm 0.4$ & 1.0 \\
\hline S256A + HU & 3 & $>3000$ & $7.6 \pm 0.9$ & none \\
\hline R657S & 6 & $>5000$ & $5.8 \pm 0.4$ & 1.0 \\
\hline R657S + HU & 6 & $>2000$ & $9.2 \pm 0.6$ & 1.5 \\
\hline F156W & 5 & $>5000$ & $73.2 \pm 2.2$ & 1.0 \\
\hline F156W + HU & 5 & $>1400$ & $54.2 \pm 3.7$ & 0.7 \\
\hline
\end{tabular}

${ }^{a}$ Total number of colonies that were analyzed by FACS in 3 to 6 independent infections.

${ }^{b}$ Determined as for Table 1.

${ }^{c}$ Calculated as follows: frequency of direct repeat deletion observed with mutant or wild-type MLV RT $\div$ frequency of direct repeat deletion observed with the same MLV $\mathrm{RT}$ in the presence of $1 \mathrm{mM} \mathrm{HU}$. Statistical analysis using the two-sample $t$ test showed that the wild-type, F156W, and R657S mutants of MLV RT displayed direct repeat deletion frequencies different from that observed in the presence of $1 \mathrm{mM} \mathrm{HU}(P<0.01)$. The direct repeat deletion frequencies obtained with the Y598V and S526A mutants of MLV RT were not significantly different in the presence or absence of $\mathrm{HU}(P>0.05)$. 


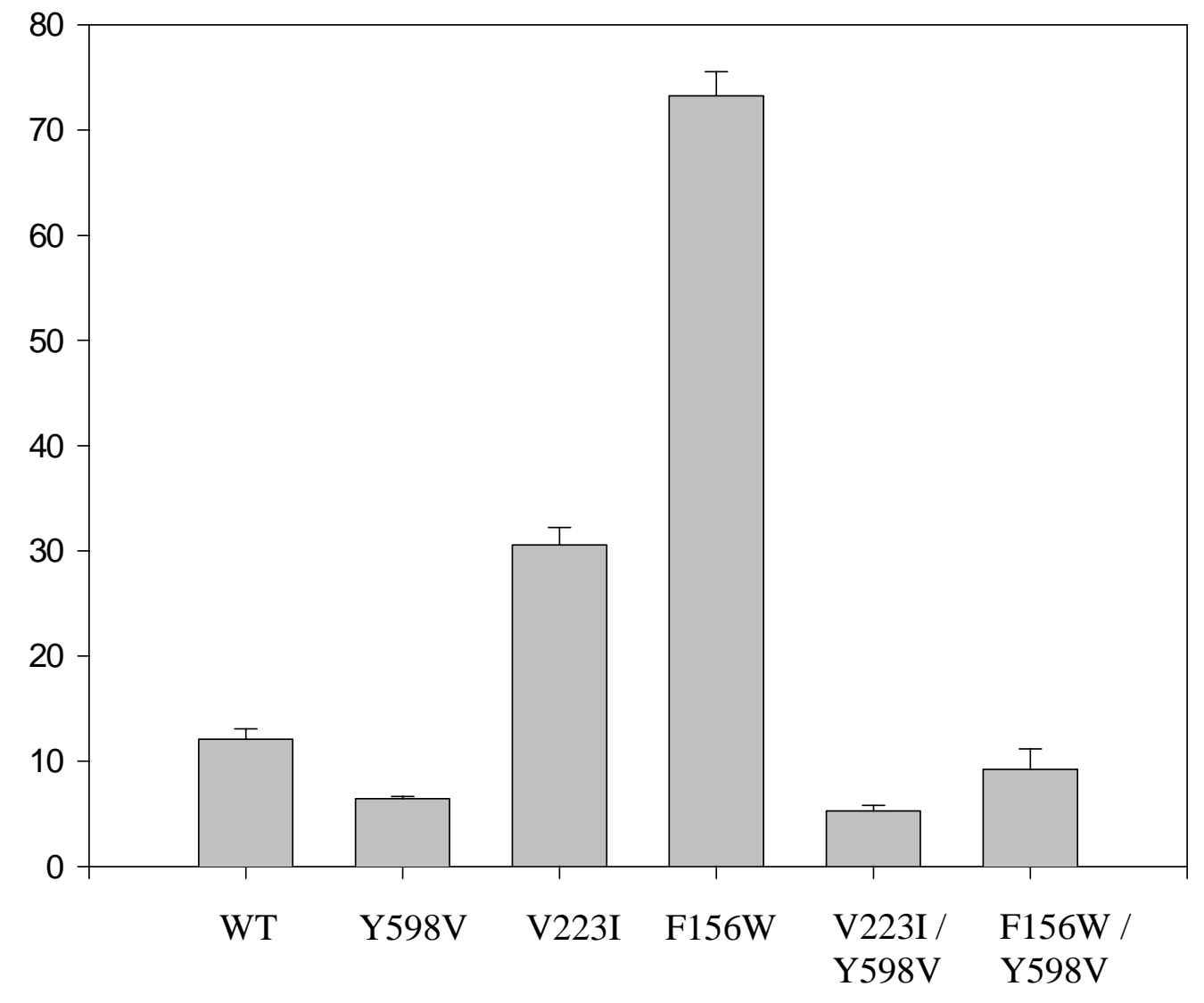

Figure 1. Effect of mutations in the MLV RT pol domain and RNase $H$ domain on the frequency of GFP reconstitution. The frequencies of GFP reconstitution were determined by FACS analysis. The results represent an average of 3 to 5 experiments, and the error bars represent the standard error of the mean. 


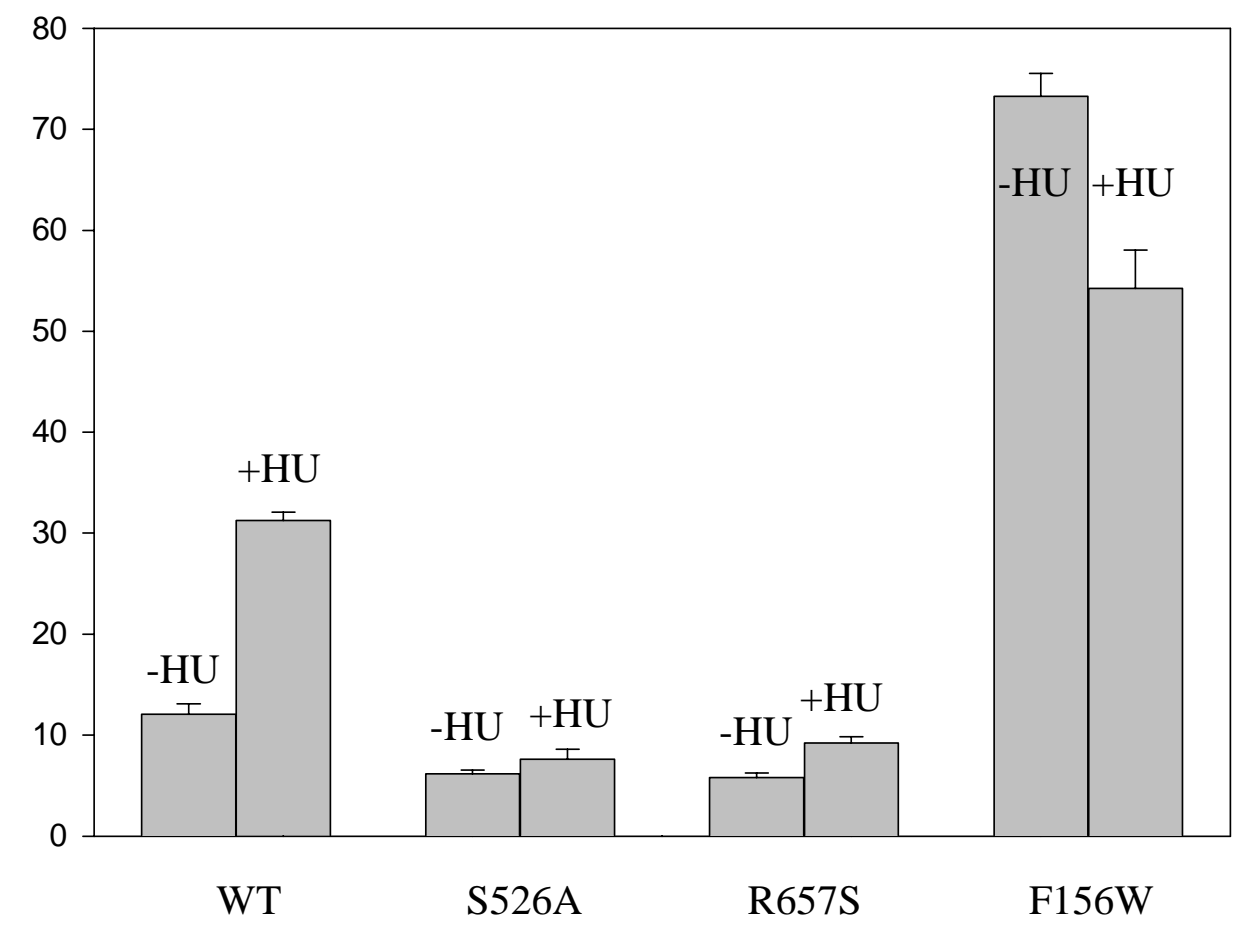

Figure 2. Effect of HU treatment on the frequencies of GFP reconstitution exhibited by the wild-type, and S526A, R657A, and F156W mutants of MLV RT. Bars represent the frequencies of GFP reconstitution observed in the absence and presence of HU treatment as indicated. The results represent an average of 3 to 6 experiments, and the error bars represent the standard error of the mean. 


\section{APPENDIX 2}

\section{CONSTRUCTION OF THE THUMB DOMAIN AND PRIMER GRIP MLV RT MUTANTS}


Thumb domain and primer grip MLV RT mutants utilized in the current study were generated by the PCR-based mutagenesis (Fig. 1). The pES4 plasmid (Chapter 3) that expresses the gag-pol MLV proteins was employed as a template for mutagenesis. First, mutagenic primers with randomized nucleotides at positions corresponding to L269, R301, G305, F309, L312, or W313 and primers outside of the BamHI sites were used to amplify overlapping fragments containing mutations at the R301 position (primer sets: 1 and 2; 3 and 4 in Fig.1). In addition, each mutagenic primer contained one or more silent mutations that resulted in the generation of a new restriction site. All mutagenic primers with corresponding restriction sites are listed in Table 1. Second, these two fragments were purified by elution from $1 \%$ agarose gels and annealed to each other by using the complementarity of the overlapping sequences. Third, the annealed fragments were extended and PCR-amplifyed by utilizing outside primers (primers 1 and 4). The resultant PCR product was digested with BamHI and gel-purified. These BamHI fragments containing mutations at positions L269, R301, G305, F309, L312, or W313 were ligated with the wild type pES4 that was digested with BamHI. Screening of the mutants was performed by utilizing new restriction sites that were generated during mutagenesis. Correct orientation of the BamHI fragment as well as the overall structure of the mutant plasmid was confirmed by restriction enzyme digestion. Additionally, the sequence between the Bam $\mathrm{HI}$ sites as well as adjacent to the Bam $\mathrm{HI}$ sites of all the mutant plasmids was also verified by sequencing. The codons for all generated mutants are summarized in Table 2. 
TABLE 1. Sequences of the primers utilized in generation of MLV RT mutants.

\begin{tabular}{|c|c|c|}
\hline Primer set & $\begin{array}{l}\text { Primer sequence (Mutated bases are shown in bold and new } \\
\text { restriction sites are underlined) }\end{array}$ & $\begin{array}{l}\text { Restriction } \\
\text { site } \\
\text { generated }\end{array}$ \\
\hline $\begin{array}{l}\text { Outside } \\
\text { primer } 1 \\
\text { (For) }\end{array}$ & 5'- GTCAACAAGCGGGTGGAAGACATCC -3' & NONE \\
\hline $\begin{array}{l}\text { Outside } \\
\text { primer } 4 \\
\text { (Rev) }\end{array}$ & 5'- CACCGGCCGACGCCAAGGTCCCAG -3' & NONE \\
\hline L269XFor & $\begin{array}{l}\text { 5'- CAGAAACAGGTCAAGTATNNSGGGTACCTT } \\
\text { CTAAAAGAGGGTC -3' }\end{array}$ & KpnI \\
\hline L269XRev & $\begin{array}{l}\text { 5'- GACCCTCTTTTAGAAGGTACCCSNNATACTT } \\
\text { GACCTGTTTCTG -3' }\end{array}$ & KpnI \\
\hline R301XFor & $\begin{array}{l}\text { 5'- CGAAGACCCCTCGACAACTANNNGAATTCCTAG } \\
\text { GGACGGC - 3' }\end{array}$ & EcoRI \\
\hline R301XRev & $\begin{array}{l}\text { 5'- GCCGTCCCTAGGAATTCNNNTAGTTGTCGAGGG } \\
\text { GTCTTCG -3' }\end{array}$ & EcoRI \\
\hline G305XFor & $\begin{array}{l}\text { 5'- CGACAACTAAGGGAATTCCTANNNACGGCAGG } \\
\text { CTTCTGTCG -3' }\end{array}$ & EcoRI \\
\hline G305XRev & $\begin{array}{l}\text { 5'- CGACAGAAGCCTGCCGTNNNTAGGAATTCCCTT } \\
\text { AGTTGTCG -3' }\end{array}$ & EcoRI \\
\hline F309XFor & $\begin{array}{l}\text { 5'- CCTAGGGACTGCAGGCVNNTGTCGCCTCTGGA } \\
\text { TC -3' }\end{array}$ & Pst $\mathrm{I}$ \\
\hline F309XRev & $\begin{array}{l}\text { 5'- GATCCAGAGGCGACANNBGCCTGCAGTCCCTA } \\
\text { GG -3' }\end{array}$ & Pst $\mathrm{I}$ \\
\hline L312XFor & $\begin{array}{l}\text { 5'- GGGACGGCAGGCTTCTGCAGANNNTGGATCCC } \\
\text { TGGGTTTGC -3' }\end{array}$ & Pst $\mathrm{I}$ \\
\hline L312XRev & $\begin{array}{l}\text { 5'- GCAAACCCAGGGATCCANNNTCTGCAGAAGCC } \\
\text { TGCCGTCCC -3' }\end{array}$ & Pst $\mathrm{I}$ \\
\hline W313XFor & $\begin{array}{l}\text { 5'- GGGACGGCAGGCTTCTGCAGACTCNNNATCCC } \\
\text { TGGGTTTGC -3' }\end{array}$ & Pst $\mathrm{I}$ \\
\hline W313XRev & $\begin{array}{l}\text { 5'- GCAAACCCAGGGATNNNGAGTCTGCAGAAGCC } \\
\text { TGCCTACCATCCC -3' }\end{array}$ & Pst $\mathrm{I}$ \\
\hline
\end{tabular}


TABLE 2. Codons of the generated mutants.

\begin{tabular}{|c|c||c|c||c|c|}
\hline Mutant & Codon & Mutant & Codon & Mutant & Codon \\
\hline \hline L269 (WT) & CTG & R301 (WT) & AGG & G305 (WT) & GGG \\
\hline L269A & GCC & R301H & CAC & G305D & GAT \\
\hline L269G & GGG & R301I & ATT & G305E & GAG \\
\hline L269H & CAC & R301L & TTG & G305F & TTC \\
\hline L269N & AAC & R301P & CCA & G305I & ATT \\
\hline L269Q & CAG & R301Q & CAG & G305N & AAT \\
\hline L269R & CGG & R301S & TCA & G305Q & CAG \\
\hline L269S & TCA & R301V & GTC & G305R & CGG \\
\hline L269T & ACG & & & G305V & GTC \\
\hline
\end{tabular}

\begin{tabular}{|c|c|c|c|c|c|}
\hline Mutant & Codon & Mutant & Codon & Mutant & Codon \\
\hline F309 (WT) & TTC & L312 (WT) & CTC & W313(WT) & TGG \\
\hline F309A & GCC & L312E & GAG & W313A & GCT \\
\hline F309H & CAC & L312R & CGG & W313D & GAT \\
\hline F309I & ATT & & & W313Q & CAA \\
\hline F309L & CTA & & & & \\
\hline F309N & AAT & & & & \\
\hline F309P & CCA & & & & \\
\hline F309T & ACA & & & & \\
\hline F309V & GTT & & & & \\
\hline F309W & TGG & & & & \\
\hline
\end{tabular}



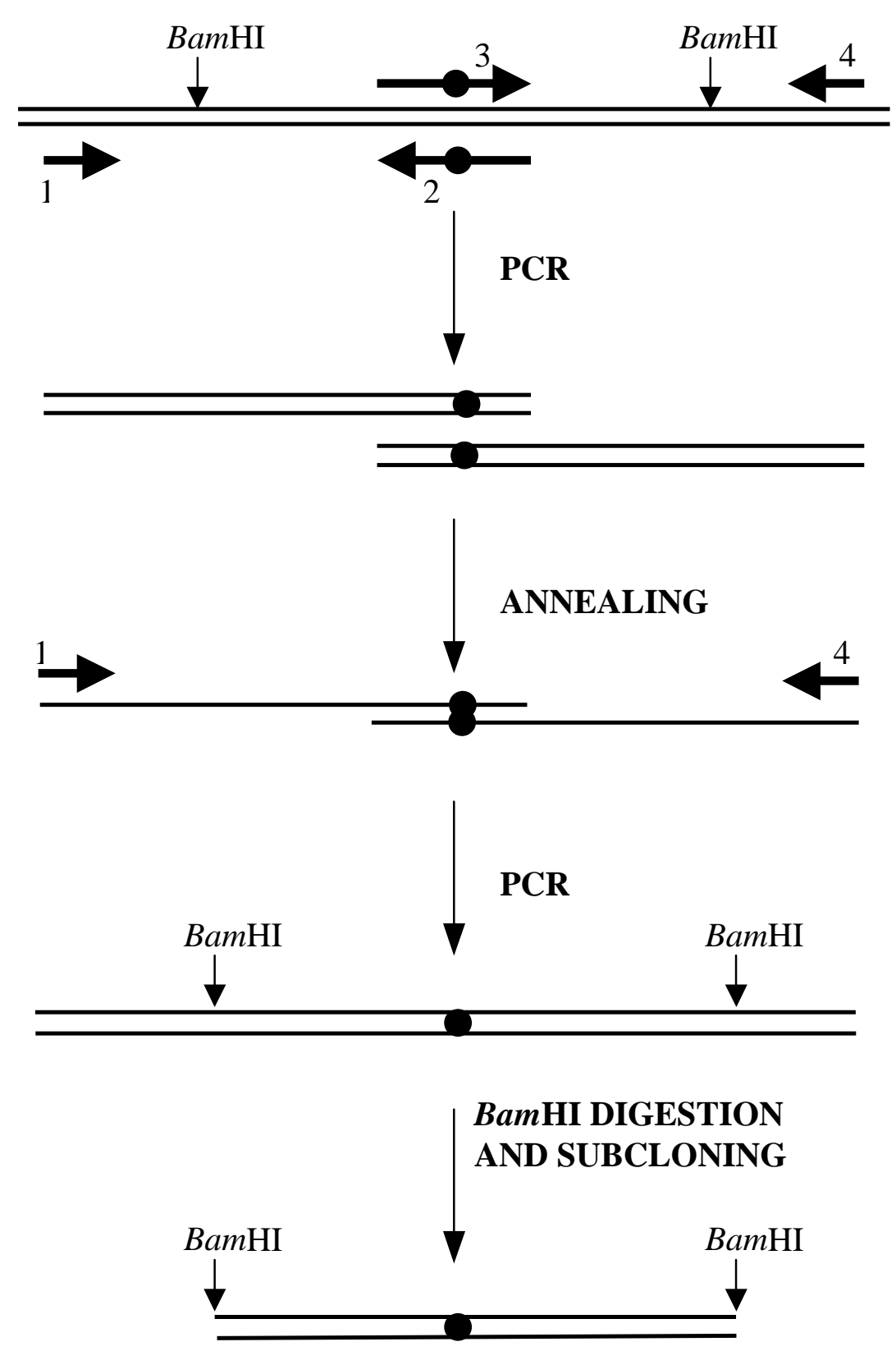

Figure 1. PCR-based mutagenesis protocol. Lines represent DNA; bold arrows represent primers; filled circles represent mutations. 


\title{
CURRICULUM VITAE
}

\author{
Evguenia Sergeevna Svarovskaia \\ Resistance Mechanisms Laboratory, Viral Mutation Section \\ HIV Drug Resistance Program, NCI \\ Building 535, Room 334, Frederick, MD 21702 \\ Telephone: (301)846-1916, Facsimile: (301)846-6013
}

\section{EDUCATION:}

1983-1985 Academician Lavrentiev's Specialized Physical and Mathematical School

Address: Pirogova, 22, Nonosibirsk, Russia, 630090

1985-1991 Novosibirsk State University, Department of Natural Sciences, Degree earned: Higher Education in Biology

Address: Pirogova, 2, Nonosibirsk, Russia, 630090

1995-1997 West Virginia University, Department of Biochemistry, Degree earned: Master of Science in Biochemistry

1995-2000 West Virginia University, Department of Biochemistry, Degree earned: Doctor of Philosophy in Biochemistry

\section{RESEARCH EXPERIENCE:}

6/88-6/91 Research Assistant at the Institute of Bioorganic Chemistry, Affiliation: Russian Academy of Sciences

Address: Prospekt Lavrientieva 3, Novosibirsk, Russia 630090

Advisor: Kravchenko, Vladimir Vitalevich

Research Project: "Regulation of translation of the distal lacZ gene in polycistronic mRNA by the ribosome stream from the proximal gene"

6/91-1/94 Assistant Research Scientist at the Institute of Biochemistry, Affiliation: Russian Academy of Medicine

Address: Timakova 2, Novosibirsk, Russia 630090

Advisor: Zagrebelny, Stanislav Nikolaevich

Research Project: "Development of overexpression System for CD4 Receptor in E.coli”

6/96-12/99 Research Assistant at West Virginia University, Department of Biochemistry

Address: Robert Bird Health Sciences Center, School of Medicine, Department of Biochemistry, Morgantown, WV 26506

Advisor: Pathak, Vinay K. 
Research Project: “ Identification of structural elements of MLV RT important for fidelity and drug-resistance"

12/99- 2000 Predoctoral fellowship at National Cancer Institute Address: HIV Drug Resistance Program, Building 535/Room 324FCRDC, Frederick, MD 21702

Advisor: Pathak, Vinay K.

Research Project: “ Identification of structural elements of MLV RT important for fidelity and template switching"

\section{PUBLICATIONS:}

Nikolenko GN; Kravchenko VV; Svarovskaia ES; Gileva IP; Likhoshvai VA; Korobko VG. Regulation of translation of the distal lacZ gene in polycistronic mRNA by the ribosome stream from the proximal gene. Bioorg. Khim. 1997, Mar;23(3):200-4.

Halvas, EK; Svarovskaia, ES; Pathak, VK. Development of an in Vivo Assay to Identify Structural Determinants in Murine Leukemia Virus Reverse Transcriptase Important for Fidelity. J. Virol., January, 2000, 74(1):312-319.

Halvas, EK; Svarovskaia, ES; Freed, E.O., Pathak, VK. Wild-Type and YMDD Mutant of Murine Leukemia Virus Reverse Transcriptase Are Resistant to 2',3'-Dideoxy-3'thiacytidine. J. Virol., July, 2000, 74(14): 6669-74. 2000,

Svarovskaia, E.S., Delviks, K.A., Hwang, C. K., and Vinay K. Pathak. Structural Determinants of Murine Leukemia Virus Reverse Transcriptase that Affect the Frequency of Template Switching. J. Virol., August, 2000, 74(15):7171-8.

Halvas, EK; Svarovskaia, ES; Pathak, VK. Murine leukemia virus reverse transcriptase dNTP binding site mutants that decrease fidelity during retroviral replication. Submitted to J.Virol, June 2000.

Svarovskaia, ES; Halvas, EK; Pattabiraman, N; Pathak, VK. Identification of Murine Leukemia Virus Minor Groove Binding Helix of the Thumb Domain and Its Role in Fidelity of Reverse Transcription inVivo. Submitted to J.Virol, August 2000.

\section{ABSTRACTS AND MEETINGS}

Jenny S. Svarovskaia and Vinay K. Pathak. 1999. Mutational analysis of the putative thumb domain of MLV RT to identify residues important for fidelity. Poster presentation at Cold Sprig Harbor Retroviruses meeting (abstract pg.74), NY

Elias K. Halvas, Evguenia S. Svarovskaia, Erin White, and Vinay K. Pathak. 1999. Structural determinants of the putative dNTP binding site in MLV RT important for 
fidelity. Poster presentation at Cold Sprig Harbor Retroviruses meeting (abstract pg.85), NY

Elias K. Halvas, Jenny S. Svarovskaia, Ronald A. Mudry, Jr., and Vinay K. Pathak. 1997. Structural determinants of MLV RT important for fidelity. Talk presentation at Cold Sprig Harbor Retroviruses meeting (abstract pg.23), NY

Elias K. Halvas, Evguenia S. Svarovskaia, Eric O. Freed, and Vinay K. Pathak Wild-Type and YMDD Mutant of Murine Leukemia Virus Reverse Transcriptase Are Resistant to 2',3'-Dideoxy-3'-thiacytidine, 2000 Poster presentation at Cold Sprig Harbor Retroviruses meeting (abstract pg.178), NY

Evguenia S. Svarovskaia, Krista A. Delviks, Carey K. Hwang, and Vinay K. Pathak. A Dynamic Copy-choice model for retroviral recombination: structural Determinants of Reverse transcriptase that Affect the Frequency of Template Switching, 2000. Talk presentation at Cold Sprig Harbor Retroviruses meeting (abstract pg.144), NY

Elias K. Halvas, Evguenia S. Svarovskaia, and Vinay K. Pathak. Murine Leukemia Virus Reverse transcriptase dNTP binding site Mutants that decrease fidelity during retroviral replication, 2000 Poster presentation at Cold Sprig Harbor Retroviruses meeting (abstract pg.179), NY 